\title{
Non-planar form factors of generic local operators via on-shell unitarity and color-kinematics duality
}

\author{
Guanda $\operatorname{Lin}^{a}$ and Gang Yang ${ }^{b, c, d, e}$ \\ ${ }^{a}$ School of Physics, Peking University, \\ Beijing 100871, China \\ ${ }^{b}$ CAS Key Laboratory of Theoretical Physics, Institute of Theoretical Physics, \\ Chinese Academy of Sciences, Beijing 100190, China \\ ${ }^{c}$ School of Fundamental Physics and Mathematical Sciences, \\ Hangzhou Institute for Advanced Study, UCAS, Hangzhou 310024, China \\ ${ }^{d}$ International Centre for Theoretical Physics Asia-Pacific, \\ Beijing/Hangzhou, China \\ e School of Physical Sciences, University of Chinese Academy of Sciences, \\ Beijing 100049, China \\ E-mail: linguandak@pku.edu.cn, yangg@itp.ac.cn
}

ABSTRACT: Form factors, as quantities involving both local operators and asymptotic particle states, contain information of both the spectrum of operators and the on-shell amplitudes. So far the studies of form factors have been mostly focused on the large $N_{c}$ planar limit, with a few exceptions of Sudakov form factors. In this paper, we discuss the systematical construction of full color dependent form factors with generic local operators. We study the color decomposition for form factors and discuss the general strategy of using on-shell unitarity cut method. As concrete applications, we compute the full twoloop non-planar minimal form factors for both half-BPS operators and non-BPS operators in the $\mathrm{SU}(2)$ sector in $\mathcal{N}=4 \mathrm{SYM}$. Another important aspect is to investigate the color-kinematics (CK) duality for form factors of high-length operators. Explicit CK dual representation is found for the two-loop half-BPS minimal form factors with arbitrary number of external legs. The full-color two-loop form factor result provides an independent check of the infrared dipole formula for two-loop $n$-point amplitudes. By extracting the UV divergences, we also reproduce the known non-planar SU(2) dilatation operator at two loops. As for the finite remainder function, interestingly, the non-planar part is found to contain a new maximally transcendental part beyond the known planar result.

Keywords: Scattering Amplitudes, Space-Time Symmetries, Supersymmetric Gauge Theory

ArXiv EPrint: 2011.06540 


\section{Contents}

1 Introduction 1

2 A review of the planar case $\quad 3$

2.1 Local gauge invariant operators 3

2.2 Tree-level amplitudes and form factors 4

2.3 Loop correction function and renormalization 5

3 Full-color structure $\quad 8$

3.1 Trace color basis 9

$\begin{array}{lll}3.2 & \text { Trivalent (DDM) color basis } & 13\end{array}$

$\begin{array}{lll}3.3 & \text { Color-kinematics duality for form factors } & 16\end{array}$

4 General discussion of non-planar unitarity 18

$\begin{array}{ll}4.1 \text { General strategy } & 18\end{array}$

4.2 Examples for unitarity construction 20

5 Full-color two-loop form factors $\quad \mathbf{2 5}$

5.1 Color decomposition of two-loop form factors 26

$\begin{array}{lll}5.2 & \text { Two-loop BPS correction } & 29\end{array}$

5.3 Two-loop SU(2) correction 35

6 Color-kinematics duality for two-loop BPS form factors 38

6.1 Six-scalar tree amplitude 38

6.2 Two-loop CK-dual representation 41

$\begin{array}{lll}7 & \text { IR, UV divergences and finite remainder } & 47\end{array}$

$\begin{array}{lll}7.1 & \text { Infrared structure with full-color } & 47\end{array}$

$\begin{array}{lll}7.2 & \text { BPS remainder } & 50\end{array}$

7.3 UV renormalization, dilatation operator and remainders 54

7.4 Comment on new features of non-planar subtractions and remainders $\quad 59$

$\begin{array}{lll}8 & \text { Summary and outlook } & 61\end{array}$

A Two-loop DDM basis $\quad 62$

$\begin{array}{ll}\text { B Non-planar features of unitarity } & 64\end{array}$

$\begin{array}{ll}\text { C Six-point amplitudes } & 67\end{array}$

$\begin{array}{ll}\text { D One-loop BPS and SU(2) form factors } & 68\end{array}$

$\begin{array}{ll}\text { E Cancellation of two-loop non-planar integrals } & 70\end{array}$ 
F.1 Details for divergence cancellation in (7.8) 71

$\begin{array}{lll}\text { F.2 Analysis for } \mathrm{SU}(2) \text { form factors } & 73\end{array}$

\section{Introduction}

The large $N_{c}$ limit (i.e. the planar limit) has been a useful approximation in the study of gauge theories [1]. In this limit, the color degrees of freedom are decoupled and irrelevant. For example, for scattering amplitudes, it is enough to consider color-ordered planar amplitudes; for anomalous dimensions or correlation functions, only single-trace operators are involved in the computation. The planar limit also induces certain symmetries beyond the full theory. An example is the planar $\mathcal{N}=4$ super-Yang-Mills theory $(\mathcal{N}=4 \mathrm{SYM})$, in which an infinite number of hidden symmetries (integrability) have been found, rendering the theory exactly solvable in the planar limit, see e.g. [2] for a review.

On the other hand, it is important to go beyond the large $N_{c}$ limit and consider the full-color contributions. In a full gauge theory, the mixing of different color structures as well as the correlation between color and spacetime degrees of freedom are expected to have significant physical effects. In the realistic QCD with $N_{c}=3$, the color-subleading parts should be indispensable for understanding the mechanism of confinement. Besides, in the phenomenological studies, non-planar contributions of amplitudes and their cross sections have also important effects.

As a useful testing ground for studying non-planar structures, we consider form factors in $\mathcal{N}=4 \mathrm{SYM}$ in this paper. They are defined as matrix elements between a local gauge invariant operator and $n$ on-shell outgoing particle states [3-9]:

$$
\mathcal{F}_{\mathcal{O}, n}=\int d^{D} x e^{-i q \cdot x}\left\langle W\left(p_{1}\right) \cdots W\left(p_{n}\right)|\mathcal{O}(x)| 0\right\rangle=(2 \pi)^{D} \delta^{(D)}\left(q-\sum_{i=1}^{n} p_{i}\right)\langle 1 \cdots n|\mathcal{O}(0)| 0\rangle,
$$

where $W\left(p_{i}\right)$ represent asymptotic on-shell states, and $\mathcal{O}(x)$ is a local operator carrying an off-shell momentum $q=\sum_{i} p_{i}$ (via the Fourier transformation). Since form factors involve both local operators and asymptotic on-shell states, they contain important information of both on-shell amplitudes and anomalous dimension of local operators. This has inspired many studies that generalize amplitudes techniques to form factors [10-33] and also use form factors to study the spectrum problem of generic operators [34-44]. A state-of-the-art pedagogical review for form factors in $\mathcal{N}=4 \mathrm{SYM}$ can be found in [45].

In the case of Sudakov form factors of the stress tensor supermultiplet in $\mathcal{N}=4 \mathrm{SYM}$, the full non-planar corrections has been considered at four and five loops [46, 47] with the help of color-kinematics duality [48, 49]. On the other hand, for form factors of generic local operators of high dimensions, the studies have so far mostly focused on the planar limit. A concrete goal of this paper is to study the non-planar form factors of generic high-length local operators in $\mathcal{N}=4 \mathrm{SYM}$. This generalization is important in the sense 
that it will allow us to probe the non-planar spectrum of general local operators, as well as the non-planar IR structure of amplitudes with generic number of external legs.

As mentioned in the beginning, the main new feature of non-planar form factors is that the color degrees of freedom must be taken into account. This generates several complications, comparing to the planar case. First, operators with multi-trace structures have to be considered. Second, the correlation between color and kinematic parts needs to be taken into account. Moreover, the kinematic part itself becomes more intricate: for example, new non-planar types of Feynman integrals will contribute. To solve these problems, two distinct approaches can be considered.

In the first approach, we apply the color decomposition for form factors to first disentangle the color and kinematic parts. Similar strategy was also considered in loop amplitudes computation, see e.g. [50-56]. For form factors of high dimension operators, an important difference comparing to amplitudes is that one can focus on a small set of density loop correction functions. Then for the non-planar kinematic parts, one can apply the on-shell unitarity method [57-59]. A generic computational strategy based on these will be described, and as concrete applications, we obtain two-loop non-planar form factors in both half-BPS and non-BPS SU(2) sectors. The advantage of the above unitarity-based method is that it does not rely on any specific symmetry of a theory (such as supersymmetry or planar integrability), thus can be used in quite general context (either in non-planar sector, or in generic theories like QCD).

As for the second approach, an important tool for non-planar construction is the colorkinematics duality [48, 49], where the entanglement between color and kinematics becomes a bonus in the construction (see [60] for an extensive review of the developments). As mentioned above, this duality has been very useful for computing the Sudakov form factors in $\mathcal{N}=4 \mathrm{SYM}[46,47]$. It would be of great interest to see if this can be applied to form factors of high-length operators which involve multiple external legs. In this paper, we make a concrete step and find a two-loop integral representation that manifests the color-kinematics duality for minimal form factors of half-BPS operators $\operatorname{tr}\left(\phi^{L}\right)$ for arbitrary $L$ (which equals to the number of external on-shell legs). It turns out that in order to satisfy the duality, certain scaleless integrals (which are zero after integration) are necessarily included.

Having the full two-loop form factor results, we are able to study several non-planar properties. First, we compute the two-loop full-color IR divergence, which provides an independent check for the dipole formula of amplitudes with arbitrary number of external legs [61, 62]. From the UV renormalization of non-BPS SU(2) form factors, we also obtain the two-loop non-planar $\mathrm{SU}(2)$ dilatation operator previously obtained in [63]. After subtracting the divergences, one can get the finite remainder function. Interestingly, we find that, beyond the known planar results [14, 36, 38], the leading transcendentality part receives a new non-planar correction.

This paper is organized as follows. In section 2, we provide a review of local gauge invariant operators and planar form factors. Section 3 concerns the generic color structures of operators and form factors. Both the trace basis and trivalent basis are studied for decomposing the color structure of form factors. A brief review for color-kinematics duality 
and its generalization to generic form factors are also given. In section 4, we explain the general strategy of computing full-color form factors with unitarity cut method, together with concrete examples. Section 5 provides the full construction and results for both two-loop half-BPS and $\mathrm{SU}(2)$ form factors. In section 6 , the representation satisfying color-kinematics duality is obtained for the two-loop half-BPS form factors. In section 7 , we discuss the non-planar properties of the two-loop IR structure, the finite remainders, and the $\mathrm{SU}(2)$ dilation operator. Finally, section 8 provides a summary and outlook. Appendix A-F contain some further discussions and several technical details.

\section{A review of the planar case}

In this section, we briefly review the gauge invariant operators and planar amplitudes and form factors. This section is mainly intended to set up the notation and provide some basic building block results that will be used in later sections. For simplicity, the discussion of this section will focus on the planar case and ignore the color degrees of freedom. The full-color extension will be discussed in detail in the next section.

\subsection{Local gauge invariant operators}

A gauge invariant local operator $\mathcal{O}(x)$ is composed of trace of gauge covariant fields at same spacetime point $x$. In $\mathcal{N}=4 \mathrm{SYM}$, the elementary fields are: gauge boson $A^{\mu}$, eight Weyl spinor $\Psi_{A}^{\alpha}, \bar{\Psi}^{\dot{\alpha} A}$ with $\alpha, \dot{\alpha}=1,2$ and $A=1,2,3,4$, and six scalars $\Phi_{I}$ with $I=1, \ldots, 6$. All the fields are in the adjoint representation of gauge group. In local operators, the gauge field $A^{\mu}$ shows up in the covariant derivative $D_{\mu}=\partial_{\mu}-i g_{\mathrm{YM}}\left[A_{\mu}, \cdot\right]$ and the field strength tensor $F^{\mu \nu}=(-i g)^{-1}\left[D^{\mu}, D^{\nu}\right]$. All the covariant fields $\mathbb{W}_{i}$ appearing in local operators can be listed as

$$
\mathbb{W} \in\left\{\Phi^{A B}, F^{\alpha \beta}, \bar{F}^{\dot{\alpha} \dot{\beta}}, \bar{\Psi}^{\dot{\alpha} A}, \Psi^{\alpha, A B C}\right\},
$$

and each field can be dressed with arbitrary number of covariant derivatives $D^{\alpha \dot{\alpha}}$. In the planar limit, one only needs to consider single trace operators which are

$$
\mathcal{O}_{L}=\operatorname{tr}\left(\mathbb{W}_{1}^{\left(n_{1}\right)} \mathbb{W}_{2}^{\left(n_{2}\right)} \ldots \mathbb{W}_{L}^{\left(n_{L}\right)}\right)
$$

where $\mathbb{W}_{i}^{(n)}=D^{n} \mathbb{W}_{i}$, and we also call $L$ the length of the operator.

An important problem regarding the local operators is to compute their dimensions. In general the canonical dimension of an operator receives quantum corrections which are called the anomalous dimensions. The anomalous dimension is captured by the dilatation operator, see e.g. [63]. Quantum correction of dilatation operator may be obtained from the computation of two-point correlation functions. In the planar limit, the eigenvalue of the dilation operators or the anomalous dimension can be computed non-perturbatively, via integrability techniques [2]. Form factors provide an alternative way to compute the dilatation operator, and as we will discuss in this paper, the on-shell method also makes it straightforward to go beyond the planar limit. 


\subsection{Tree-level amplitudes and form factors}

In contrast to correlation functions of local operators, amplitudes and form factors in $\mathcal{N}=4$ SYM contain on-shell asymptotic states which can be conveniently represented by Nair's on-shell superfield [64]:

$$
W(p, \eta)=g_{+}(p)+\eta^{A} \bar{\psi}_{A}(p)+\frac{\eta^{A} \eta^{B}}{2 !} \phi_{A B}(p)+\frac{\epsilon_{A B C D} \eta^{A} \eta^{B} \eta^{C}}{3 !} \psi^{D}(p)+\eta^{1} \eta^{2} \eta^{3} \eta^{4} g_{-}(p)
$$

To clarify our notation, we use $\mathbb{W}_{i}$ to represent off-shell fields in operators, and $W_{i}\left(p_{i}, \eta_{i}\right)$ to denote on-shell super fields. We also denote $\mathcal{W}_{i}\left(p_{i}\right)$ for certain component of $W_{i}\left(p_{i}, \eta_{i}\right)$, i.e. $\mathcal{W}_{i} \in\left\{\phi_{i}, \psi_{i}, \bar{\psi}_{i}, g_{i, \pm}\right\}$.

Tree-level amplitudes. Amplitudes are gauge invariant quantities only depending on on-shell states and are given as $\left\langle W\left(p_{1}\right) \cdots W\left(p_{n}\right) \mid 0\right\rangle$. Here we briefly review some results of planar color-ordered tree amplitudes. See e.g. [65, 66] for more detailed introductions.

Firstly, the $n$-point tree-level super MHV amplitudes take the well-known Parke-Taylor form [67]:

$$
\hat{\mathcal{A}}_{n}^{(0), \mathrm{MHV}}(1, \ldots, n)=\frac{\delta^{(4)}(P) \delta^{(8)}(Q)}{\langle 12\rangle\langle 23\rangle \cdots\langle n 1\rangle},
$$

where $P^{\alpha \dot{\alpha}}=\sum_{i=1}^{n} \lambda^{\alpha} \tilde{\lambda}^{\dot{\alpha}}$ and $Q^{\alpha, A}=\sum_{i=1}^{n} \lambda_{i}^{\alpha} \eta_{i}^{A}$, and $\overline{\mathrm{MHV}}$ amplitudes can be derived by conjugate. Here we denote super amplitudes $\hat{\mathcal{A}}$ with a "hat" to stress their dependence on Grassmann variables, and amplitudes $\mathcal{A}$ without "hat" are bosonic components with only kinematic dependence. The four-point pure scalar amplitudes $\mathcal{A}_{4}^{(0)}\left(p_{1}^{X}, p_{2}^{Y}, p_{3}^{\bar{X}}, p_{4}^{\bar{Y}}\right)$ can be obtained by extracting the $\eta_{1}^{1} \eta_{1}^{2} \eta_{2}^{1} \eta_{2}^{3} \eta_{3}^{3} \eta_{3}^{4} \eta_{4}^{2} \eta_{4}^{4}$ component from (2.4):

$$
\mathcal{A}_{4}^{(0)}\left(p_{1}^{X}, p_{2}^{Y}, p_{3}^{\bar{X}}, p_{4}^{\bar{Y}}\right)=1,
$$

where $X=\phi_{12}, Y=\phi_{13}$ and $\bar{X}=\phi_{34}, \bar{Y}=\phi_{42}$. And similarly,

$$
\mathcal{A}_{4}^{(0)}\left(p_{1}^{X}, p_{2}^{Y}, p_{3}^{\bar{Y}}, p_{4}^{\bar{X}}\right)=-\frac{\langle 13\rangle\langle 24\rangle}{\langle 14\rangle\langle 23\rangle}=-\left(1+\frac{s_{12}}{s_{14}}\right) .
$$

Furthermore, for higher point amplitudes, we also have $\mathrm{N}^{k} \mathrm{MHV}$ amplitudes. They can be, for example, computed via BCFW on-shell recursion [68] or MHV vertex expansion [69] methods. In our two-loop computation, we encounter 6-point NMHV amplitudes of scalars. All of them can be conveniently expressed in a form with Mandelstam variables, for example,

$$
\mathcal{A}_{6}^{(0)}\left(p_{1}^{X}, p_{2}^{X}, p_{3}^{Y}, p_{4}^{\bar{X}}, p_{5}^{\bar{X}}, p_{6}^{\bar{Y}}\right)=-\frac{1}{s_{234}},
$$

where we use the notation $s_{i j \ldots k}=\left(p_{i}+p_{j}+\ldots+p_{k}\right)^{2}$. Other six-scalar amplitudes that are needed in the two-loop computation are listed in appendix $\mathrm{C}$.

Tree-level form factors. Next we consider tree-level form factors. The simplest class of form factors are the minimal tree-level form factors, $\mathcal{F}_{\mathcal{O}_{L}, n}^{(0)}$ with $n=L$, in which the external legs are in a one-to-one correspondence with the covariant fields in the operators, which are related via Wick contraction and LSZ reduction. The correspondence, or say a 


\begin{tabular}{|c|c|c|}
\hline off-shell fields $\mathbb{W}$ & on-shell states $\mathcal{W}_{o}$ & helicity spinor variables \\
\hline$F^{\dot{\alpha} \dot{\beta}}$ & $\stackrel{g_{+}}{\longrightarrow}$ & $\tilde{\lambda}^{\dot{\alpha}} \tilde{\lambda}^{\dot{\beta}}$ \\
$\bar{\Psi}^{\dot{\alpha} A}$ & $\stackrel{\bar{\psi}_{A}}{\longrightarrow}$ & $\tilde{\lambda}^{\dot{\alpha}} \eta^{A}$ \\
$\Phi^{A B}$ & $\stackrel{\phi_{A B}}{\longrightarrow}$ & $\eta^{A} \eta^{B}$ \\
$\Psi^{\alpha A B C}$ & $\stackrel{q_{A B C}}{\longrightarrow}$ & $\lambda^{\alpha} \eta^{A} \eta^{B} \eta^{C}$ \\
$F^{\alpha \beta}$ & $\stackrel{g_{-}}{p_{\alpha \dot{\alpha}}}$ & $\lambda^{\alpha} \lambda^{\beta} \eta^{1} \eta^{2} \eta^{3} \eta^{4}$ \\
$D^{\alpha \dot{\alpha}}$ & $\stackrel{\lambda^{\alpha} \tilde{\lambda}^{\dot{\alpha}}}{ }$ \\
\hline
\end{tabular}

Table 1. Correspondence between local operators and minimal form factors.

\begin{tabular}{|c|c|}
\hline external states & next to minimal form factors $\mathcal{F}_{L+1}^{(0)}$ \\
\hline$\cdots \phi_{i, A B} g_{i+1}^{+} \phi_{i+2, C D} \cdots$ & $\cdots \eta_{i}^{A} \eta_{i}^{B} \frac{\langle i i+2\rangle}{\langle i i+1\rangle\langle i+1 i+2\rangle} \eta_{i+2}^{C} \eta_{i+2}^{D} \cdots$ \\
$\cdots \phi_{i, A B} g_{i+1,1234}^{-} \phi_{i+2, C D} \cdots$ & $\cdots \eta_{i}^{A} \eta_{i}^{B} \frac{[i+2]}{[i i+1][i+1 i+2]} \eta_{i+1}^{1} \eta_{i+1}^{2} \eta_{i+1}^{3} \eta_{i+1}^{4} \eta_{i+2}^{C} \eta_{i+2}^{D} \cdots$ \\
$\cdots \bar{\psi}_{i, A} \bar{\psi}_{i+1, B} \phi_{i+1, C D} \cdots$ & $\cdots \frac{1}{\langle i i+1\rangle}\left(\eta_{i}^{A} \eta_{i+1}^{B}-\eta_{i}^{B} \eta_{i+1}^{A}\right) \eta_{i+2}^{C} \eta_{i+2}^{D} \cdots$ \\
$\cdots \psi_{i}^{A^{\prime}} \psi_{i+1}^{B^{\prime}} \phi_{i+1, C D} \cdots$ & $\cdots \frac{-1}{[i i+1]}\left(\bar{\eta}_{i, A^{\prime}} \bar{\eta}_{i+1, B^{\prime}}-\bar{\eta}_{i, B^{\prime}} \bar{\eta}_{i+1, A^{\prime}}\right) \eta_{i+2}^{C} \eta_{i+2}^{D} \cdots$ \\
\hline
\end{tabular}

Table 2. Density of next-to-minimal tree-level form factors as building blocks in unitarity, where the involved fields in the operators $\operatorname{are} \operatorname{tr}\left(\ldots \Phi^{A B} \Phi^{C D} \ldots\right)[36]$.

dictionary translating operators to minimal form factors in spinor helicity formalism, can be summarized in table $1[34,70,71]$.

To obtain a minimal tree-level form factor from a given operator, for example, for $\mathcal{O}=\operatorname{tr}\left(F^{3}\right)=\operatorname{tr}\left(F_{\dot{\beta}}^{\dot{\alpha}} F_{\dot{\gamma}}^{\dot{\beta}} F_{\dot{\alpha}}^{\dot{\gamma}}\right)$, one can use the map in table 1 and obtain $\mathcal{F}_{\mathcal{O}, 3}^{(0)}$ as

$$
\mathcal{F}_{\mathcal{O}, 3}^{(0)}\left(1^{g_{+}}, 2^{g_{+}}, 3^{g_{+}}\right)=\left(\tilde{\lambda}_{1}^{\dot{\alpha}} \tilde{\lambda}_{1 \dot{\beta}}\right)\left(\tilde{\lambda}_{2}^{\dot{\beta}} \tilde{\lambda}_{2 \dot{\gamma}}\right)\left(\tilde{\lambda}_{3}^{\dot{\gamma}} \tilde{\lambda}_{3 \dot{\alpha}}\right)+\text { cyc.perm. }(1,2,3)=3[12][23][31]
$$

For more general non-minimal tree-level form factors, we introduce next ${ }^{k}$-to-minimal tree-level form factor $\mathcal{F}_{\mathcal{O}_{L}, n}^{(0)}$, which contains a length- $L$ operator and $n=L+k$ on-shell legs. Non-minimal tree-level form factors also have simplicities such as the Parke-Taylor like structure and can be computed using amplitudes techniques $[6,8]$ (see also methods using twistor formalism [20-23]). To construct two-loop form factors, one will need nextto-minimal tree-level form factors. In particular, for the next-to-minimal tree-level form factors of operators composed of pure scalar fields, the building blocks are listed in table 2, see [36] for more details.

\subsection{Loop correction function and renormalization}

In this subsection, we consider planar form factors at loop level. We introduce loop correction function and discuss its connection to the renormalization. The loop expansion for 


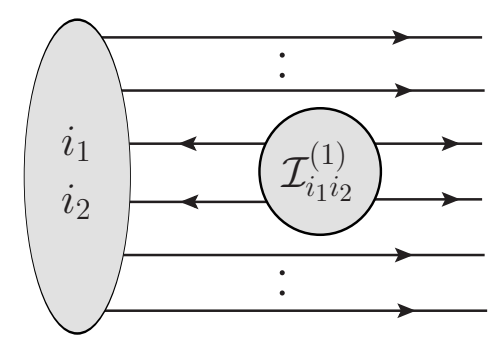

(a) One-loop density.

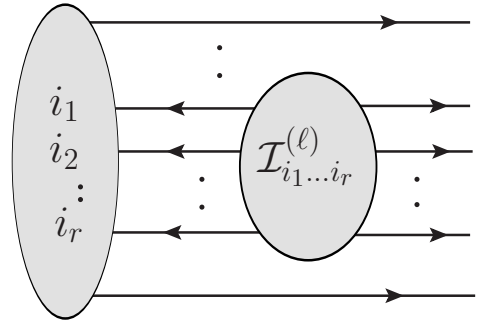

(b) Higher-loop density functions.

Figure 1. Diagrammatic representation for loop correction density functions.

planar minimal form factors is defined as

$$
\mathcal{F}=\sum_{l=0}^{\infty} g^{2 \ell} \mathcal{F}^{(\ell)}, \quad \text { with } \quad g^{2}=\frac{g_{\mathrm{YM}}^{2} N_{\mathrm{c}}}{(4 \pi)^{2}}\left(4 \pi e^{-\gamma_{\mathrm{E}}}\right)^{\epsilon} .
$$

Loop correction density function. An $\ell$-loop form factor of generic operators may be expressed in terms of a loop correction function $\mathcal{I}^{(\ell)}$ acting on tree level form factors

$$
\mathcal{F}_{\mathcal{O}}^{(\ell)}=\mathcal{I}^{(\ell)} \cdot \mathcal{F}_{\mathcal{O}}^{(0)}
$$

The full loop correction function $\mathcal{I}^{(\ell)}$ can be decomposed in terms of loop density functions, $\mathcal{I}_{i_{1} i_{2} \ldots i_{r}}^{(\ell)}$, as represented by figure 1 . The subindices " $i_{1} i_{2} \ldots i_{r}$ " denotes the sites of the operators that the density function acting on. The number $r$ is named the interaction range. At one loop, it is obvious that the interaction range is two. At $\ell$-loop, the maximal (connected) interaction range is $\ell+1$. In the planar limit, $i_{1} \ldots i_{r}$ should be adjacent: $i, i+1, \ldots, i+(r-1)$. The complete planar form factor is given by summing over density functions of all external momenta plus summing over their insertion on all possible sites in the operators. For example, the one-loop form factor can be written as

$$
\mathcal{F}_{\mathcal{O}}^{(1)}=\sum_{i} \mathcal{I}_{i(i+1)}^{(1)} \cdot \mathcal{F}_{\mathcal{O}}^{(0)}
$$

Since the operator may contain different type of fields, it should be clear that in general the loop correction functions are matrices acting in the field space. For example, the above one-loop density should be understood as in

$$
\begin{aligned}
& \mathcal{F}_{\mathcal{O}_{\mathcal{W}_{i} \mathcal{W}_{i+1}}^{(1)}}\left(p_{k}^{\widetilde{\mathcal{W}}_{k}}, p_{k+1}^{\widetilde{\mathcal{W}}_{k+1}}\right)=\left(\mathcal{I}^{(1)}\right)_{\mathcal{W}_{i} \mathcal{W}_{i+1}}^{\widetilde{\mathcal{W}}_{k+1} \widetilde{\mathcal{W}}_{k+1}}\left(p_{k}, p_{k+1}\right)\left(\widetilde{\mathcal{W}}_{k} \frac{\delta}{\delta \mathcal{W}_{i}} \widetilde{\mathcal{W}}_{k+1} \frac{\delta}{\delta \mathcal{W}_{i+1}}\right) \mathcal{F}_{\mathcal{O}_{\mathcal{W}_{i} \mathcal{W}_{i+1}}^{(0)}} \\
& =\left(\mathcal{I}^{(1)}\right)_{\mathcal{W}_{i} \mathcal{W}_{i+1}}^{\widetilde{\mathcal{W}}_{k} \widetilde{\mathcal{W}}_{k+1}}\left(p_{k}, p_{k+1}\right) \mathcal{F}_{\mathcal{O}_{\widetilde{\mathcal{W}}_{k}}^{(0)} \widetilde{\mathcal{W}}_{k+1}}\left(p_{k}, p_{k+1}\right)
\end{aligned}
$$

where $\mathcal{O}_{\mathcal{W}_{i} \mathcal{W}_{i+1}}$ is to stress that the $i$-th and $(i+1)$-th field in operator $\mathcal{O}$ are $\mathcal{W}_{i}$ and $\mathcal{W}_{i+1}{ }^{1}$ and " $\mathcal{W}_{k}, \widetilde{\mathcal{W}}_{k+1}$ " label the fields of external on-shell legs. A more precise definition for the full non-planar form factor will be given in the next section, see (3.8).

\footnotetext{
${ }^{1}$ Here we do not distinguish on-shell and off-shell fields, since they are related by the dictionary given in table 1.
} 
Having this structure in mind, one can focus on the density functions in the computation of loop form factors, and it is enough to calculate all matrix elements such as $\left(\mathcal{I}^{(1)}\right)_{\mathcal{W}_{i} \mathcal{W}_{j}}^{\widetilde{\mathcal{W}}_{k} \widetilde{\mathcal{W}}_{l}}$ in the one-loop case. For full-color form factors, one needs to dress loop corrections with color indices as well, which will be discussed in next section. As we will discuss shortly, the matrix form is related to the fact that different operators are mixed with each other via quantum corrections. Through renormalization, one can extract the operator mixing information using form factors.

Renormalization, operator mixing and dilatation operator. Loop correction functions of form factors of general operators contain both ultraviolet (UV) and infrared (IR) divergences. The IR structure is well understood and can be subtracted systematically, while the UV divergences require renormalization and result in operator mixing.

The renormalization of operators can be expressed as

$$
\mathcal{O}_{I}^{\text {ren }}=\sum_{J} \mathcal{Z}_{I}^{J} \mathcal{O}_{J}^{\text {bare }}
$$

where $\mathcal{Z}$ is the renormalization constant matrix. Renormalization of form factors can be expressed likewise

$$
\mathcal{F}_{\mathcal{O}_{I}}^{\mathrm{ren}}=\sum_{J} \mathcal{Z}_{I}^{J} \mathcal{F}_{\mathcal{O}_{J}}^{\text {bare }}
$$

More explicitly, we have

$$
\mathcal{F}_{\mathcal{O}_{I}}^{\text {ren }}=\sum_{J} \mathcal{Z}_{I}^{J} \mathcal{F}_{\mathcal{O}_{J}}^{\text {bare }}=\sum_{J, K} \mathcal{Z}_{I}^{J}\left(\mathcal{I}^{\text {bare }}\right)_{J}^{K} \mathcal{F}_{\mathcal{O}_{K}}^{(0)} \equiv \sum_{J}\left(\mathcal{I}^{\text {ren }}\right)_{I}^{J} \mathcal{F}_{\mathcal{O}_{J}}
$$

The UV divergence of $\mathcal{Z}_{I}^{J} \mathcal{I}_{J}^{\text {bare }}$ should cancel order by order. Up to two-loop order, we have

$$
\begin{aligned}
& \mathcal{I}^{(1) \text {,ren }}=\mathcal{I}^{(1), \text { bare }}+\mathcal{Z}^{(1)}, \\
& \mathcal{I}^{(2), \text { ren }}=\mathcal{I}^{(2), \text { bare }}+\mathcal{Z}^{(2)}+\mathcal{Z}^{(1)} \mathcal{I}^{(1), \text { bare }} .
\end{aligned}
$$

We stress that the product of loop corrections and renormalization constants should be taken as matrix products. For example, the one-loop square should be understood as

$$
\left(\mathcal{I}^{(1)}\right)^{2} \mathcal{F}_{\mathcal{O}_{I}}^{(0)}=\sum_{J, K}\left(\mathcal{I}^{(1)}\right)_{I}^{J}\left(\mathcal{I}^{(1)}\right)_{J}^{K} \mathcal{F}_{\mathcal{O}_{K}}^{(0)}
$$

The renormalization can be expressed in the density form as well. Take the one-loop case as an example:

$$
\mathcal{Z}^{(1)}=\sum_{i} \mathcal{Z}_{i(i+1)}^{(1)}
$$

By imposing UV cancellation at density level

$$
\mathcal{I}_{i(i+1)}^{(1), \text { ren }}=\mathcal{I}_{i(i+1)}^{(1), \text { bare }}+\mathcal{Z}_{i(i+1)}^{(1)}
$$

one can extract the renormalization constant density $\left(\mathcal{Z}^{(1)}\right)_{\mathcal{W}_{i} \mathcal{W}_{j}}^{\mathcal{W}_{j} \mathcal{W}_{l}}$. Examples of the $\mathrm{SO}(6)$ and $\operatorname{SL}(2)$ sector can be found in [45]. 
Given the renormalization constant $\mathcal{Z}$, it is straightforward to calculate the anomalous dilatation operator $\delta \mathbb{D} \equiv \mathbb{D}-\mathbb{D}_{0}$, where $\mathbb{D}_{0}$ corresponds to classical dimensions, as ${ }^{2}$

$$
\delta \mathrm{D}=2 \epsilon g^{2} \frac{\partial}{\partial g^{2}} \mathcal{Z}=\sum_{\ell=1}^{\infty} g^{2 \ell} \mathbb{D}^{(\ell)} .
$$

Up to two loops, $\mathbb{D}^{(\ell)}$ can be derived from renormalization constant as

$$
\begin{aligned}
& \mathrm{D}^{(1)}=2 \epsilon \mathcal{Z}^{(1)}, \\
& \mathbb{D}^{(2)}=4 \epsilon\left[\mathcal{Z}^{(2)}-\frac{1}{2}\left(\mathcal{Z}^{(1)}\right)^{2}\right] .
\end{aligned}
$$

Finally, by diagonalizing the dilatation operator, one can obtain the eigenstates and their eigenvalues are the anomalous dimensions.

\section{Full-color structure}

To develop approaches towards non-planar form factors, a clear understanding of the color structure is required. In section 3.1 and 3.2, we take the decomposition approach and consider color decomposition for form factors, so that the color and kinematic parts are separated in a well controlled way. In section 3.3, alternatively, we discuss the colorkinematics duality which imposes a powerful symmetry on the kinematic part by using color factors, and generalize the duality to form factors of high-length operators.

To be concrete, we will mostly consider $\mathrm{SU}\left(N_{\mathrm{c}}\right)$ gauge group for simplicity. In $\mathcal{N}=4$ SYM, all fields are in the adjoint representation: $\mathbb{W}=\mathbb{W}^{a} T^{a}$, where $T^{a}, a=1, \ldots, N_{\mathrm{c}}^{2}-1$, are the generators of $\mathrm{SU}\left(N_{c}\right)$ and satisfy commutation relations

$$
\left[T^{a}, T^{b}\right]=i \sqrt{2} f^{a b c} T^{c}=\tilde{f}^{a b c} T^{c}, \quad \tilde{f}^{a b c}=\operatorname{tr}\left(T^{a} T^{b} T^{c}\right)-\operatorname{tr}\left(T^{a} T^{c} T^{b}\right) .
$$

When contracting color indices, we need the completeness relations:

$$
\sum_{a=1}^{N_{\mathrm{c}}^{2}-1}\left(T^{a}\right)_{\mathrm{x}_{1}}^{\mathrm{y}_{1}}\left(T^{a}\right)_{\mathrm{x}_{2}}^{\mathrm{y}_{2}}=\delta_{\mathrm{x}_{1}}^{\mathrm{y}_{2}} \delta_{\mathrm{x}_{2}}^{\mathrm{y}_{1}}-\frac{1}{N_{c}} \delta_{\mathrm{x}_{1}}^{\mathrm{y}_{1}} \delta_{\mathrm{x}_{2}}^{\mathrm{y}_{2}}
$$

where $\mathrm{x}_{i}, \mathrm{y}_{i}=1, \ldots N_{c}$ are (anti)fundamental indices. We choose normalization: $\operatorname{tr}\left(T^{a} T^{b}\right)=\delta^{a b}$.

For generic loop expansion at non-planar level, we use the color-independent coupling $\tilde{g}=g / \sqrt{N_{c}}$. The loop expansion of full-color form factors is defined as

$$
\boldsymbol{F}=\sum_{l=0}^{\infty} \tilde{g}^{2 \ell} \boldsymbol{F}^{(\ell)}, \quad \text { with } \quad \tilde{g}^{2}=\frac{g_{\mathrm{YM}}^{2}}{(4 \pi)^{2}}\left(4 \pi e^{-\gamma_{\mathrm{E}}}\right)^{\epsilon}
$$

\footnotetext{
${ }^{2}$ For special subsectors, such as the $\mathrm{SU}(2)$ sector, we only need to consider even powers of $g$ in the expansion. The odd power of $g$ expansion corresponds to the "length changing effect", see e.g. [72]. We will not consider such case in this paper.
} 


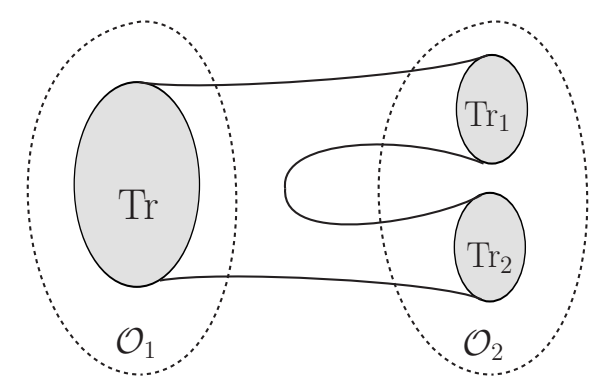

Figure 2. At non-planar level, local operators with different numbers of color traces can mix with each other. This diagram illustrates the interaction between a single-trace operator and a double-trace operator, which leads to a non-trivial two-point correlation function between the two operators.

\subsection{Trace color basis}

We first consider the color structure in terms of trace basis.

Color structure of operators. For local operators, the color structures are naturally defined in terms of color traces. In the planar limit, one can confine oneself in single-trace operators as given in (2.2), as interactions between fields of different traces are suppressed by $1 / N_{\mathrm{c}}$. For full non-planar considerations, it is necessary to consider multi-trace operators. We can define a generic operator as

$$
\mathcal{O}_{L}=C_{\mathcal{O}}\left(a_{1}, \ldots a_{L}\right) \mathbb{W}_{1}^{a_{1},\left(n_{1}\right)} \mathbb{W}_{2}^{a_{2},\left(n_{2}\right)} \ldots \mathbb{W}_{L}^{a_{L},\left(n_{L}\right)}
$$

where the color factor $C_{\mathcal{O}}$ in general contains multiple traces and is given as a product of the following terms:

$$
\operatorname{tr}(\mathbb{1})=N_{c}, \operatorname{tr}\left(T^{a_{1}} T^{a_{2}}\right), \ldots, \operatorname{tr}\left(T^{a_{1}} T^{a_{2}} \ldots T^{a_{m}}\right), \ldots
$$

One can characterize the color structure of an operator by the number of traces and the length of each trace.

Non-planar corrections can lead to an interaction between operators with different color structure. In figure 2, we illustrate a two-point function between a single-trace and a double-trace operator, which never contribute in the planar limit. Consequently, only after considering operators of all color structures can one determine the full non-planar anomalous dimensions (as the eigenstates of dilatation mixing matrix). When increasing the length (i.e. the number of fields) of the operators, the number of inequivalent trace factors can grow very fast. Comparing to the planar case, this increases the number of operators as well as the complexity of the mixing matrices significantly.

Trace basis for form factors. The trace basis can be also used for form factors, and the corresponding color factors can be given as products of terms in (3.5) as well. It should be clear that different products of (3.5) are linear independent. Here it is important to note the difference between the trace structure of local operators and form factors: the 
operators are always color singlet, while the color factor of a form factor is a $\mathrm{SU}\left(N_{\mathrm{c}}\right)$ tensor with free color indices carried by the external on-shell states.

We denote the trace basis by $\mathcal{T}_{k}$, and one can make a color decomposition of form factors as:

$$
\boldsymbol{F}_{\mathcal{O}, n}^{(\ell)}=\sum_{k} \mathcal{T}_{k}\left[\mathcal{F}_{\mathcal{O}, n}^{(\ell)}\right]_{\mathcal{T}_{k}},
$$

where $\mathcal{T}_{k}$ are product of traces with total length $n$, and the color-stripped form factors are denoted as $\left[\mathcal{F}_{\mathcal{O}, n}^{(\ell)}\right]_{\mathcal{T}_{k}}$. We use bold italic character $\boldsymbol{F}(\boldsymbol{A})$ to denote the full-color form factors (amplitudes). The linear independence of $\mathcal{T}_{k}$ also implies an important fact: each $\left[\mathcal{F}_{\mathcal{O}, n}^{(\ell)}\right]_{\mathcal{T}_{k}}$ should be a gauge invariant quantity and have appropriate physical factorization and proper IR/UV structure.

As an example for the trace color factors, for the minimal form factor of a length- 4 operator, the trace color basis $\left\{\mathcal{T}_{k}\right\}$ can be chosen as

$$
N_{\mathrm{c}}^{m} \operatorname{tr}\left(T^{a_{1}} T^{a_{\sigma(2)}} T^{a_{\sigma(3)}} T^{a_{\sigma(4)}}\right), \quad N_{\mathrm{c}}^{n} \operatorname{tr}\left(T^{a_{1}} T^{\left.a_{\tau(2)}\right)} \operatorname{tr}\left(T^{a_{\tau(3)}} T^{a_{\tau(4)}}\right), \quad \text { with } \sigma \in S_{3}, \tau \in Z_{3},\right.
$$

where powers of $N_{c}$ depend on the number of loops $\ell$ in general. In this example, number of traces plus the leading power of $N_{c}$ equals to $\ell+1$ as the loop number increases.

Definition of full-color loop correction. Following section 2.3, we discuss colordressed loop correction functions. Here we use trace basis to illustrate the definition and concepts, and the discussion will also apply to other color bases such as DDM basis given in the next subsection.

As in the planar case, we can write the full-color loop form factor as

$$
\boldsymbol{F}^{(\ell)}=\boldsymbol{I}^{(\ell)} \cdot \boldsymbol{F}^{(0)} .
$$

Furthermore, $\boldsymbol{I}^{(\ell)}$ can be decomposed in terms density functions. We label $\ell$-loop density for color-dressed loop correction as $\boldsymbol{I}_{i_{1} i_{2} \cdots i_{r}}^{(\ell)}$, where the subscript $i_{i} i_{2} \cdots i_{r}$ denote the sites of the operator on which the density acting on, as in figure 1. Importantly, the sites are not required to be adjacent in the full-color context, unlike the planar case.

Now let us consider the one-loop case in detail. The full one-loop form factor can be expressed in terms of density function as:

$$
\begin{aligned}
& \boldsymbol{F}_{\mathcal{O}\left(\mathcal{W}_{1} \ldots \mathcal{W}_{L}\right)}^{(1)}\left(p_{1}^{\widetilde{\mathcal{W}}_{1}}, \ldots, p_{L}^{\widetilde{\mathcal{W}}_{L}}\right) \\
&=\sum_{k \neq l, i \neq j}\left(\boldsymbol{I}^{(1)}\right)_{\mathcal{W}_{i} \mathcal{W}_{j}}^{\widetilde{\mathcal{W}}_{l} \widetilde{\mathcal{W}}_{l}}\left(p_{k}, p_{l}\right)\left(\widetilde{\mathcal{W}}_{k} \frac{\delta}{\delta \mathcal{W}_{i}} \widetilde{\mathcal{W}}_{l} \frac{\delta}{\delta \mathcal{W}_{j}}\right)\left(p_{k} \frac{\delta}{\delta q_{i}} p_{l} \frac{\delta}{\delta q_{j}}\right) \\
& \times \frac{1}{S_{(i j)}^{(k l)}} \sum_{\{m, n\}} \prod_{m \neq k, l ; n \neq i, j}\left(p_{m} \frac{\delta}{\delta q_{n}} \delta_{\mathcal{W}_{n}}^{\widetilde{\mathcal{W}}_{m}}\right) \cdot \boldsymbol{F}_{\mathcal{O}\left(\mathcal{W}_{1} \ldots \mathcal{W}_{L}\right)}^{(0)}\left(q_{1}^{\mathcal{W}_{1}}, \ldots, q_{L}^{\mathcal{W}_{L}}\right) .
\end{aligned}
$$

The first line comes from the contribution from intrinsic one-loop corrections (which is a range- 2 interaction). And the second line is the trivial tree factors that involve remaining $(L-2)$ legs, where the $(L-2)$ states $\left(\left\{\widetilde{\mathcal{W}}_{m}\right\}\right)$ should be in a one-to-one correspondence with the $(L-2)$ fields $\left(\left\{\mathcal{W}_{n}\right\}\right)$ in the operator, and $S_{(i j)}^{(k l)}$ account for the possible symmetry 
factors in the case when there are identical fields. Obviously, to compute one-loop form factor, one only needs to focus on the density function $\left(\boldsymbol{I}^{(1)}\right)_{\mathcal{W}_{i} \mathcal{W}_{j}}^{\widetilde{\mathcal{W}}_{k}} \widetilde{\widetilde{\mathcal{W}}}_{\text {. This discussion should }}$ be straightforward to be generalized to higher loop form factors.

Color structure of loop density functions. Similar to (3.6), one can also perform a color decomposition for the density function and expand $\boldsymbol{I}^{(\ell)}$ as

$$
\boldsymbol{I}_{i_{1} i_{2} \cdots i_{r}}^{(\ell)}\left(p_{j_{1}}, \ldots, p_{j_{r}}\right)=\sum_{\alpha} \check{\mathcal{C}}_{\alpha}\left[\left(\mathcal{I}^{(\ell)}\right)_{\mathcal{W}_{i_{1}} \cdots \mathcal{W}_{i_{r}}}^{\widetilde{\mathcal{W}}_{j_{1} \cdots \widetilde{\mathcal{W}}_{j_{r}}}}\left(p_{j_{1}}, \ldots, p_{j_{r}}\right)\right]_{\check{\mathcal{C}}_{\alpha}},
$$

where $\check{\mathcal{C}}_{\alpha}$ is the color factor which should be understood as an "operator" acting on color space of sites $i_{1} \cdots i_{r}$. A one-loop example of color decomposition of $\boldsymbol{I}_{i j}^{(1)}\left(p_{k}, p_{l}\right)$ is

$$
\boldsymbol{I}_{i j}^{(1)}\left(p_{k}, p_{l}\right)=\sum_{\alpha} \check{\mathcal{C}}_{\alpha}\left[\left(\mathcal{I}^{(1)}\right)_{\mathcal{W}_{i} \mathcal{W}_{j}}^{\widetilde{\mathcal{W}}_{l} \widetilde{\mathcal{W}}_{l}}\left(p_{k}, p_{l}\right)\right]_{\check{\mathcal{C}}_{\alpha}} .
$$

And $\check{\mathcal{C}}_{\alpha}$ should be understood as

$$
\check{\mathcal{C}}_{\alpha}=\check{\mathcal{C}}_{\alpha}\left(T^{a_{k}}, T^{a_{l}}, \frac{\delta}{\delta T^{a_{i}}}, \frac{\delta}{\delta T^{a_{j}}}\right),
$$

where it plays a role of annihilating two color charges $a_{i}, a_{j}$ in the operator (or say tree level form factor) and regenerate them for the external legs, similar to the action $\left(\widetilde{\mathcal{W}}_{k} \frac{\delta}{\delta \mathcal{W}_{i}}\right)\left(\widetilde{\mathcal{W}}_{l} \frac{\delta}{\delta \mathcal{W}_{j}}\right) \cdot \boldsymbol{F}_{\mathcal{O}_{\mathcal{W}_{i}} \mathcal{W}_{j}}^{(0)}$ in the field space in (3.8).

Let us explain these notations with concrete examples in trace basis. First, we define variation in color space operator, or color variation, as $\check{T}^{a} \equiv \frac{\delta}{\delta T^{a}}$, and its action on color structure reads ${ }^{3}$

$$
\left(\check{T}^{a}\right)_{\mathrm{z}}^{\mathrm{w}} \operatorname{tr}\left(T^{a_{1}} T^{a_{2}} \cdots T^{a_{L}}\right)=\sum_{i=1}^{L} \delta^{a a_{i}}\left(T^{a_{i+1}}\right)_{\mathrm{z}}^{\mathrm{x}_{i+2}}\left(T^{a_{i+2}}\right)_{\mathrm{x}_{i+2}}^{\mathrm{x}_{i+3}} \cdots\left(T^{a_{i-1}}\right)_{\mathrm{x}_{i-1}}^{\mathrm{w}},
$$

where repeated upper and lower indices means summation form 1 to $N_{\mathrm{c}}$. We can define generalized color trace factors by inserting variation operators $\check{T}^{a}$, for example, $\operatorname{tr}\left(\check{T}^{a} T^{b} T^{c}\right) \equiv\left(T^{b}\right)_{\mathrm{y}_{2}}^{\mathrm{y}_{3}}\left(T^{c}\right)_{\mathrm{y}_{3}}^{\mathrm{y}_{1}}\left(\check{T}^{a}\right)_{\mathrm{y}_{1}}^{\mathrm{y}_{2}}$. Another example is the product of two traces

$$
\begin{aligned}
\operatorname{tr}\left(\check{T}^{a} T^{b} T^{c}\right) \operatorname{tr}\left(T^{a_{1}} T^{a_{2}} \cdots T^{a_{L}}\right) & =\left(T^{b}\right)_{\mathrm{y}_{2}}^{\mathrm{y}_{3}}\left(T^{c}\right)_{\mathrm{y}_{3}}^{\mathrm{y}_{1}} \sum_{i=1}^{L} \delta^{a a_{i}}\left(T^{a_{i+1}}\right)_{\mathrm{y}_{1}}^{\mathrm{x}_{i+2}} \cdots\left(T^{a_{i-1}}\right)_{\mathrm{x}_{i-1}}^{\mathrm{y}_{2}} \\
& =\sum_{i=1}^{L} \delta^{a a_{i}} \operatorname{tr}\left(T^{a_{1}} \cdots T^{a_{i-1}} T^{b} T^{c} T^{a_{i+1}} \cdots T^{a_{L}}\right) .
\end{aligned}
$$

For simplicity of notations, we abbreviate $\operatorname{tr}\left(T^{a_{1}} \cdots T^{a_{L}}\right)$ as $\operatorname{tr}\left(a_{1} \cdots a_{L}\right)$, while $\operatorname{tr}(\check{a} b c)$ is short for $\operatorname{tr}\left(\check{T}^{a} T^{b} T^{c}\right)$.

\footnotetext{
${ }^{3}$ Similar definition has been used in e.g. [63]. Here we do not consider normal order because we forbid $\check{a}$ act on $T^{a}$ with in the same trace.
} 
After clarifying the notations, we go back to (3.10) with $j=i+1$ for simplicity. There are six color factors in the trace basis and the density function can be expanded as:

$$
\boldsymbol{I}_{i(i+1)}^{(1)}\left(p_{1}, p_{2}\right)=\sum_{k=1}^{6} \check{\mathcal{T}}_{k}\left[\left(\mathcal{I}^{(1)}\right)_{\mathcal{W}_{i} \mathcal{W}_{i+1}}^{\widetilde{\mathcal{W}}_{1} \widetilde{\mathcal{W}}_{2}}\left(p_{1}, p_{2}\right)\right]_{\check{\mathcal{T}}_{k}},
$$

where the color basis $\left\{\check{\mathcal{T}}_{k}\right\}$ can be chosen as

$$
\sigma \cdot \operatorname{tr}\left(a_{1} a_{2} \check{a}_{l_{2}} \check{a}_{l_{1}}\right) \equiv \operatorname{tr}\left(a_{1} \sigma\left(a_{2}\right) \sigma\left(\check{a}_{l_{2}}\right) \sigma\left(\check{a}_{l_{1}}\right)\right), \quad \text { with } \sigma \in S_{3}
$$

with $\check{a}_{l_{1}}, \check{a}_{l_{2}}$ acting on color indices in the $i$-th and $(i+1)$-th sites, for example,

$$
\check{\mathcal{T}}_{1} \cdot \mathcal{C}_{\mathcal{O}}=\operatorname{tr}\left(a_{1} a_{2} \check{a}_{l_{2}} \check{a}_{l_{1}}\right) \operatorname{tr}(\cdots \underbrace{a_{l_{1}} a_{l_{2}}}_{\text {sites } i, i+1} \cdots)=N_{c} \operatorname{tr}\left(\cdots a_{1} a_{2} \cdots\right)+\cdots,
$$

which contains a planar color factor $N_{c} \operatorname{tr}\left(\cdots a_{1} a_{2} \cdots\right)$. We stress that to clearly distinguish internal and external lines, we will re-name the internal lines as $l_{1}, \ldots, l_{r}$, so that the $\delta / \delta T^{a_{i}}$ in (3.11) should be replaced by $\delta / \delta T^{a_{l_{1}}}$. In later discussion, for the convenience of notation, we usually omit the label of fields for $\mathcal{I}$ and make an abbreviation as:

$$
\mathcal{I}^{(1)}\left(p_{1}, p_{2}\right) \equiv\left(\mathcal{I}^{(1)}\right)_{\mathcal{W}_{i} \mathcal{W}_{i+1}}^{\widetilde{\mathcal{W}}_{1} \widetilde{\mathcal{W}}_{2}}\left(p_{1}, p_{2}\right)
$$

and we use similar abbreviations for general cases.

Before discussing other color basis, let us make two important comments:

1. We have introduced two kinds of pictures to deal with loop level color structure for form factors. In the first picture, one can decompose the full-color loop form factor to a set of color basis as (3.6), which is similar to the usual treatment for amplitudes. In the second picture, we introduce the loop density functions and consider the color decomposition at density level as (3.9). One can always translate one picture to another, but they are suitable in different situations. For short length operators, such as $L=2,3$, the simplicity of color structure implies that the first picture is better. When considering form factors of generic high-length operators, the second picture is certainly much more convenient.

2. As previously mentioned, in the non-planar discussion, one has to include operators with different color structures, such as multiple-trace operators. Using the picture of loop density functions, one makes a "decoupling" between loop corrections and the detailed structure of operators. In the computation of density functions, the structure of operator is not important (and therefore one can use single trace operator for simplicity). Only when computing the complete form factor, one needs to act the density functions on particular operators (which is a combinatoric computation); and comparing to single trace operators, the extra complexity of multiple-trace operators is only at the combinatoric level (but not in a quantum loop computation). 


\subsection{Trivalent (DDM) color basis}

The trace basis we discussed in the last subsection has the important advantage that the corresponding kinematic coefficients are form factors with particular ordering of on-shell particles. Consequently, they have much simpler kinematic properties and are easier to construct via unitarity cuts.

On the other hand, it is also clear that the trace basis for form factors (and also for amplitudes) is usually overcomplete, namely, the physical form factors or amplitudes live in a smaller subspace of the space spanned by trace basis. This is obvious from the Feynman diagram origin of the amplitudes or form factors, where the color factors are given by product of trivalent structure constants $f^{a b c}$. By solving linear Jacobi relations for all trivalent graphs, a minimal set of color basis in terms of $f$ 's can be obtained [73]. We will call this alternative basis as trivalent color basis, or DDM basis.

Since the trace basis is larger than the DDM basis, the kinematic factors in the trace basis are not all independent but satisfy certain linear relations. At tree level, this precisely explains the origin of tree-level $\mathrm{U}(1)$ decoupling and KK relations [74]. The advantage of the trivalent color basis is that it can reduce the number of independent kinematic parts significantly, making the reconstruction of full-color quantities simpler.

To distinguish different bases, we use following different notations:

$$
\text { Trace basis : } \quad \boldsymbol{F}=\sum_{i=1}^{d(\mathcal{T})} \mathcal{T}_{i} \times[\mathcal{F}]_{\mathcal{T}_{i}}, \quad \text { DDM basis : } \quad \boldsymbol{F}=\sum_{i=1}^{d(\mathcal{D})} \mathcal{D}_{i} \times[\mathcal{F}]_{\mathcal{D}_{i}},
$$

where $[\mathcal{F}]_{\mathcal{C}_{i}}$ is the kinematic coefficient of color factor $\mathcal{C}_{i}$, and $d(\mathcal{C})$ is the dimension of the color basis.

Below we first review the amplitude case and then generalize it to form factors.

A review for amplitudes. Consider the four-point tree amplitude $\boldsymbol{A}_{4}^{(0)}$ as an example. In the trace basis, there are six elements:

$$
\left\{\mathcal{T}_{\sigma}\right\}=\left\{\operatorname{tr}\left(T^{a_{1}} T^{a_{\sigma(2)}} T^{a_{\sigma(3)}} T^{a_{\sigma(4)}}\right)\right\}_{\sigma \in S_{3}} .
$$

On the other hand, the trivalent DDM basis contains only two elements, and

$$
\boldsymbol{A}_{4}^{(0)}=\mathcal{D}_{1}\left[\mathcal{A}_{4}^{(0)}\right]_{\mathcal{D}_{1}}+\mathcal{D}_{2}\left[\mathcal{A}_{4}^{(0)}\right]_{\mathcal{D}_{2}}
$$

where

$$
\left\{\mathcal{D}_{1}, \mathcal{D}_{2}\right\}=\left\{\tilde{f}^{a_{1} a_{2} \times} \tilde{f}^{\mathrm{x} a_{3} a_{4}}, \tilde{f}^{a_{1} a_{3} \mathrm{x}} \tilde{f}^{\mathrm{x} a_{2} a_{4}}\right\} .
$$

Expressing the DDM basis in trace basis one obtains

$$
\begin{aligned}
\boldsymbol{A}_{4}^{(0)}= & {\left[\mathcal{A}_{4}^{(0)}\right]_{\mathcal{D}_{1}}\left(\operatorname{tr}\left(a_{1} a_{2} a_{3} a_{4}\right)+\text { rev. }\right)+\left[\mathcal{A}_{4}^{(0)}\right]_{\mathcal{D}_{2}}\left(\operatorname{tr}\left(a_{1} a_{3} a_{2} a_{4}\right)+\text { rev. }\right) } \\
& +\left(-\left[\mathcal{A}_{4}^{(0)}\right]_{\mathcal{D}_{1}}-\left[\mathcal{A}_{4}^{(0)}\right]_{\mathcal{D}_{2}}\right)\left(\operatorname{tr}\left(a_{1} a_{2} a_{4} a_{3}\right)+\text { rev. }\right)
\end{aligned}
$$

where 'rev.' means reverse of trace ordering. From (3.22), it is obvious that the six colorordered amplitudes has only two independent components, and one can easily read out the 
reflection symmetry and $\mathrm{U}(1)$ decoupling relation (which is equivalent to the KK-relation at four points):

$$
\begin{aligned}
& \mathcal{A}_{n}^{(0)}(1, \cdots, n)=(-1)^{n} \mathcal{A}_{n}^{(0)}(n, \cdots, 1), \quad \text { for } n=4, \\
& \mathcal{A}_{4}^{(0)}(i, j, k, l)+\mathcal{A}_{4}^{(0)}(i, k, l, j)+\mathcal{A}_{4}^{(0)}(i, k, j, l)=0,
\end{aligned}
$$

in which $\mathcal{A}_{4}^{(0)}(i, j, k, l)$ is a color-ordered amplitude with color factor $\operatorname{tr}\left(a_{i} a_{j} a_{k} a_{l}\right)$.

More generally, for $n$-point tree amplitudes the DDM basis can be given as [73]

$$
\begin{aligned}
\left\{\mathcal{D}_{\mathrm{DDM}}(\sigma)\right\}_{\sigma \in S_{n-2}} & =\left\{\sigma \cdot \tilde{f}^{a_{1} a_{2} \mathrm{x}_{1}} \tilde{f}^{\mathrm{x}_{1} a_{3} \mathrm{x}_{2}} \ldots \tilde{f}^{\mathrm{x}_{n-3} a_{n-1} a_{n}}\right\} \\
& \equiv\left\{\tilde{f}^{a_{1} a_{\sigma(2)} \mathrm{x}_{1}} \tilde{f}^{\mathrm{x}_{1} a_{\sigma(3)} \mathrm{x}_{2}} \ldots \tilde{f}^{\mathrm{x}_{n-3} a_{\sigma(n-1)} a_{n}}\right\} .
\end{aligned}
$$

As a result, there are only $(n-2)$ ! linear independent partial amplitudes, which leads to linear relations between partial amplitudes in trace basis. By rewriting the DDM basis into trace basis, one can reproduce the KK relations for color-ordered amplitudes [74]:

$$
\mathcal{A}_{n}^{(0)}(1,\{\alpha\}, n,\{\beta\})=(-1)^{n_{\beta}} \sum_{\sigma \in\{\alpha\} \uplus\{\beta\}^{T}} \mathcal{A}_{n}^{(0)}(1, \sigma, n),
$$

where $\{\beta\}^{T}$ is $\{\beta\}$ with the ordering reversed, and $\{\alpha\} \amalg\{\beta\}^{T}$ is the shuffle product of $\{\alpha\}$ and $\{\beta\}^{T}$, i.e. all possible shuffle of the merged list preserving the order of $\{\alpha\}$ and $\{\beta\}^{T}$ within the merged list.

In summary, the idea of DDM basis is to express color factors using (linear) independent chain of structure constant. If we consider the constraints from group theory only, then DDM basis is the minimal basis for color factors in general gauge theories with fields in adjoint representation. This basis reduces the number of matrix elements that one needs to calculate, and can be used to derive KK-like relations for partial amplitudes in the trace basis. Similar relations have been considered at higher loop level and applied for computing amplitudes, see e.g. [50-56].

Trivalent basis and KK-relations for form factors. We now consider the trivalent basis for form factors. Comparing to amplitudes, the main new feature of form factors is the appearance of a local operator.

We consider first the tree level case. The minimal tree form factors have the same trace structure as the operator and the color decomposition is trivial. Thus, only for non-minimal tree form factors one needs to consider trivalent basis. The construction of tree-level trivalent basis is straightforward by sewing trivalent basis for tree-level amplitudes on tree-level form factors.

Given the trivalent basis, one can derive KK-like relation for general color-ordered form factors. The essential difference between tree-level KK relations for general form factors and for amplitudes is that the order of fields is fixed for a given operator, and partial form factors appearing in KK relations must obey this order. For example, for $\mathcal{O}=\operatorname{tr}\left(\mathbf{W}_{1} \ldots \mathbf{W}_{L}\right)$ and external states $\left\{\mathcal{W}_{1}\left(p_{1}\right), \ldots, \mathcal{W}_{L}\left(p_{L}\right), g_{+}\left(p_{L+1}\right), g_{+}\left(p_{L+2}\right)\right\}$, the 


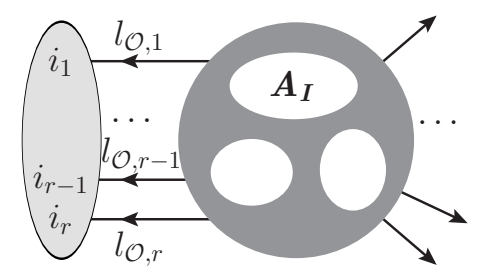

(a) Loop correction density.

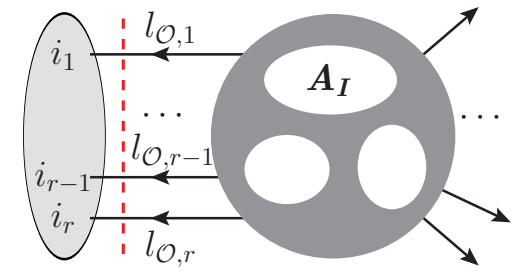

(b) Amplitudes from setting $l_{\mathcal{O}, i}$ on-shell.

Figure 3. Diagrammatic representation for loop correction density and corresponding amplitudes.

order of $\mathcal{W}_{i}$ must be $(1,2, \ldots, L)$ in partial form factors (in trace basis). The corresponding KK relations for the color-ordered form factors then read

$$
\begin{aligned}
\sum_{\sigma \in\left\{p_{3}, \ldots, p_{L}\right\} \uplus\left\{p_{L+2}, p_{L+1}\right\}} \mathcal{F}_{L+2}\left(p_{2}, \sigma, p_{1}\right) & =\mathcal{F}_{L+2}\left(p_{2},\left\{p_{3}, \ldots, p_{L}\right\}, p_{1},\left\{p_{L+1}, p_{L+2}\right\}\right), \\
\sum_{\sigma \in\left\{p_{L+2}\right\} \uplus\left\{p_{1}, \ldots, p_{i}, p_{L+1}, p_{i+1}, \ldots, p_{L}\right\}} \mathcal{F}_{L+2}(\sigma) & =0 .
\end{aligned}
$$

When it comes to loop-level form factors, the trivalent part of the color factor is provided by an amplitude part that is sewed to the operator, such as shown in figure 3 . Therefore, it is natural to consider the density picture. ${ }^{4}$ At $\ell$ loops, the possible interaction range $r$ is $2 \leq r \leq(\ell+1)$. Consider an $\ell$-loop minimal form factor, the range- $r$ density is given by sewing a $(\ell+1-r)$-loop $(2 r)$-point amplitude to the minimal tree form factor. To construct trivalent basis for the form factor density, the problem is reduced to select proper linear independent color factors for this cut amplitude.

Consider for example the one-loop density. The interaction range is $r=2$. For a minimal form factor, the cut amplitude is a four-point tree amplitude. Clearly, one can choose two trivalent basis for the one-loop density function:

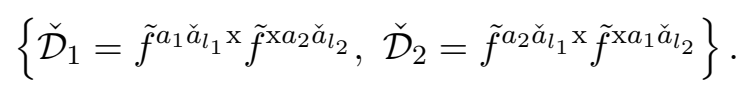

so that the minimal one-loop correction density has the form

$$
\boldsymbol{I}_{i_{1} i_{2}}^{(1)}\left(p_{1}, p_{2}\right)=\sum_{i=1}^{2} \check{\mathcal{D}}_{i}\left[\mathcal{I}^{(1)}\left(p_{1}, p_{2}\right)\right]_{\check{\mathcal{D}}_{i}} .
$$

It is natural to map between this basis and the trace basis expansion (3.14) in the previous section. For example, $\check{\mathcal{D}}_{1}=$ $\left(\operatorname{tr}\left(a_{1} a_{2} \check{a}_{l_{2}} \check{a}_{l_{1}}\right)+\operatorname{tr}\left(a_{1} \check{a}_{l_{1}} \check{a}_{l_{2}} a_{2}\right)-\operatorname{tr}\left(a_{1} \check{a}_{l_{2}} a_{2} \check{a}_{l_{1}}\right)-\operatorname{tr}\left(a_{1} \check{a}_{l_{2}} a_{2} \check{a}_{l_{1}}\right)\right)$ and one can easily find out relations between kinematic parts, such as

$$
\left[\mathcal{I}^{(1)}\right]_{\check{\mathcal{D}}_{1}}=\left[\mathcal{I}^{(1)}\right]_{\check{\mathcal{T}}_{1}}
$$

The above discussion can be generalized to higher loops in a similar way. We will discuss two-loop minimal form factors in detail in section 5, see also appendix A.

\footnotetext{
${ }^{4}$ In the context of loop-level form factors, we only focus on those "connected" densities, i.e. those can not be trivially separated as two disconnected lower-loop densities.
} 


\subsection{Color-kinematics duality for form factors}

A remarkable observation made by Bern, Carrasco and Johansson $[48,49]$ reveals that the kinematic numerators of perturbative amplitudes may satisfy similar Jacobi relations of color factors. An extensive review of the developments can be found in [60]. Here we first briefly review this duality between color and kinematics in the context of amplitudes, then we generalize to the loop density functions of form factors.

The color-kinematics duality (CK duality) states that it is possible to rearrange the perturbative amplitudes, such that the Jacobi relations satisfied by color factors of cubic graphs also apply for the kinematic numerators of same diagrams. More concretely, an $\ell$-loop amplitude with full-color dependence can be given in the form

$$
\boldsymbol{A}_{\mathcal{O}, n}^{(\ell)}=\sum_{\sigma_{n}} \sum_{\Gamma_{i}} \int \prod_{\alpha=1}^{\ell} \mathrm{d}^{D} l_{\alpha} \frac{1}{S_{i}} \frac{\mathcal{C}_{i} N_{i}}{\prod_{a} d_{i, a}},
$$

where for a given trivalent graph denoted by $\Gamma_{i}, S_{i}$ is the symmetry factor of the graph, $\mathcal{C}_{i}$ is the color factor, and $d_{i, a}$ are the propagators. The kinematic numerators, $N_{i}$, are the only objects that are not determined by $\Gamma_{i}$ and contain the intrinsic physical information of the amplitudes. The CK duality requires the numerators $N_{i}$ to take a special form so that they satisfy the same Jacobi relations for the color factors:

$$
\mathcal{C}_{i}=\mathcal{C}_{j}+\mathcal{C}_{k} \quad \rightarrow \quad N_{i}=N_{j}+N_{k}
$$

We will refer the kinematic Jacobi relation as dual Jacobi relations.

Color-kinematics duality is very powerful in computing full-color amplitudes at high loops, see e.g. [49, 75-85]. Dual Jacobi relations, together with other constraints, result in significant simplification: if the CK-dual form exists, only a small subset of numerators, called master numerators, are necessary to consider. One can start with making an ansatz for the master numerators which are enough to determine the full integrand, and then employ various physical constrains such as unitarity check to determine the ansatz.

To apply the similar procedure to calculate full-color form factors, one should take into account the effect of local operators. For the case of Sudakov form factors of short length operators, CK duality has been achieved up to five loops [46, 47]. On the other hand, whether CK duality is true for form factors of high-length operators is still unknown.

Since we want to study operators with general length, it is convenient to consider the density picture. In this case, color factors for form factors are determined in terms of two aspects: the trivalent topology of the sub-amplitude, and the local operator where the trivalent topology "springs" from. More concretely, following figure 3, for an operator $\mathcal{O}=\operatorname{tr}\left(\mathbf{W}_{1} \ldots \mathbf{W}_{L}\right){ }^{5}$ a range- $r$ interaction includes trivalent topology connected to the $i_{1}, i_{2}, \ldots, i_{r}$-th fields, with $i_{\alpha} \in 1, \ldots, L$, so the color factor from a given loop correction density $\boldsymbol{I}_{i_{1} i_{2} \cdots i_{r}}^{(\ell)}=\boldsymbol{I}_{\left\{i_{\alpha}\right\}}^{(\ell)}$ is given as

$$
\mathcal{C}\left(\left\{i_{\alpha}\right\}, \Gamma^{\boldsymbol{A}}\right)=\check{\mathcal{C}}_{\Gamma^{A}}^{\left\{i_{\alpha}\right\}} \cdot \mathcal{C}_{\mathcal{O}}=\left(\prod \tilde{f} \prod_{\alpha} \tilde{f}^{\mathrm{x}_{\alpha} \mathrm{y}_{\alpha} \check{a}_{i_{\alpha}}}\right) \cdot \operatorname{tr}\left(a_{1} \ldots a_{i_{\alpha}} \ldots a_{i_{L}}\right),
$$

\footnotetext{
${ }^{5}$ We use a single trace operator to clarify our notation, and the principle applies for general operators.
} 


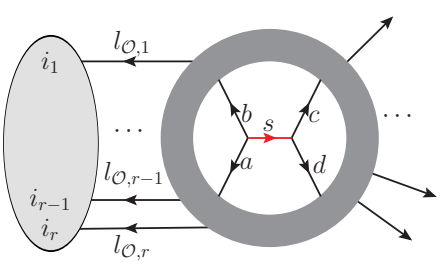

(a) s-channel

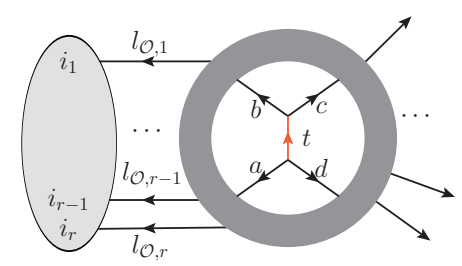

(b) t-channel

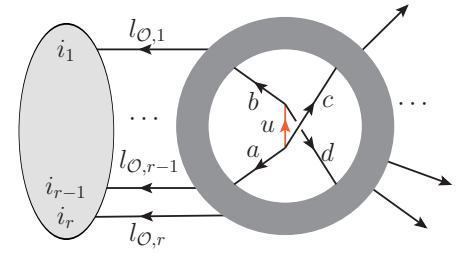

(c) $u$-channel

Figure 4. Three topologies related by Jacobi identities.

where $\Gamma^{\boldsymbol{A}}$ are the trivalent topologies of sub-amplitudes. It is worthwhile stressing that for general operators, color factors $\mathcal{C}\left(\left\{i_{\alpha}\right\}, \Gamma^{\boldsymbol{A}}\right)$ with different $\left\{i_{\alpha}\right\}$ are linearly independent with each other. Therefore, we do not expect there are Jacobi relations between color factors of different $\left\{i_{\alpha}\right\}$. As a corollary, interaction with different ranges should be viewed as decoupled in the context of dual Jacobi relations.

The above discussion shows that for form factor densities, one can first classify the loop corrections according the interaction ranges, and then for each range $r$, consider Jacobi identities between color factors with the same range interaction $\left\{i_{\alpha}\right\}$. Similar to (3.31), one can write the loop density as

$$
\boldsymbol{I}_{\left\{i_{\alpha}\right\}}^{(\ell)}=\sum_{\sigma} \sum_{\Gamma_{j}^{A}} \int \prod_{\alpha=1}^{\ell} \mathrm{d}^{D} l_{\alpha} \frac{1}{S_{j}^{\left\{i_{\alpha}\right\}}} \frac{\check{\mathcal{C}}_{j}^{\left\{i_{\alpha}\right\}} N_{j}^{\left\{i_{\alpha}\right\}}}{\prod_{a} d_{j, a}},
$$

where $\check{\mathcal{C}}_{j}^{\left\{i_{\alpha}\right\}}$ is the color factor from the sub-amplitudes topology $\Gamma_{j}^{\boldsymbol{A}}$.

For any propagators not directly connected to the operators, labeled by red line in figure 4(a), one can rotate it into the other two channels as in figure 4(b) and 4(c), which we call $s, t$ and $u$ channel respectively. The color factors of these three diagrams are

$$
\mathcal{C}_{s}^{\left\{i_{\alpha}\right\}}=\tilde{f}^{a b s} \tilde{f}^{c d s} \prod \tilde{f} \cdot \mathcal{C}_{\mathcal{O}}, \quad \mathcal{C}_{t}^{\left\{i_{\alpha}\right\}}=\tilde{f}^{b c t} \tilde{f}^{d a t} \prod \tilde{f} \cdot \mathcal{C}_{\mathcal{O}}, \quad \mathcal{C}_{u}^{\left\{i_{\alpha}\right\}}=\tilde{f}^{a c u} \tilde{f}^{b d u} \prod \tilde{f} \cdot \mathcal{C}_{\mathcal{O}},
$$

where $\prod \tilde{f}$ comes from other vertices which are identical in these three diagrams, $\mathcal{C}_{\mathcal{O}}$ is color factor of operator $\mathcal{O}$. The color factors of densities $\check{\mathcal{C}}_{j}^{\left\{i_{\alpha}\right\}}$, defined in $\mathcal{C}_{s}^{\left\{i_{\alpha}\right\}}=\check{\mathcal{C}}_{j}^{\left\{i_{\alpha}\right\}} \cdot \mathcal{C}_{\mathcal{O}}$, satisfy the corresponding Jacobi relation

$$
\check{\mathcal{C}}_{s}^{\left\{i_{\alpha}\right\}}=\check{\mathcal{C}}_{t}^{\left\{i_{\alpha}\right\}}+\check{\mathcal{C}}_{u}^{\left\{i_{\alpha}\right\}} .
$$

Thus, the kinematic numerators are expected to satisfy the dual Jacobi relation:

$$
N_{s}^{\left\{i_{\alpha}\right\}}=N_{t}^{\left\{i_{\alpha}\right\}}+N_{u}^{\left\{i_{\alpha}\right\}},
$$

resulting in a large set of linear relations between kinematic numerators.

For supersymmetric theories, such as $\mathcal{N}=4 \mathrm{SYM}$, it is possible to impose further constraints on numerators. For quantities with good UV behavior, such as scattering amplitudes or BPS form factors, supersymmetric cancellation may lead to the following simplifications: (1) one may eliminate topology with sub-bubble/triangle, except when 
those sub-bubble/triangle are directly connected to the operators, and (2) one may impose constraint on the power of loop momenta which is useful to reduce the size of ansatz. Though, one should be cautious that these conditions may not always be satisfied and may be relaxed according to specific circumstances, see section 6 for more discussions.

\section{General discussion of non-planar unitarity}

In this section, we describe the general strategy of computing full-color form factors with (generalized) unitarity cut method [57-59]. Unitarity cut serves to construct perturbative amplitudes or form factors from on-shell "building blocks" which have lower loops or points. Here cut refers to setting certain (off-shell) internal lines to be on-shell

$$
\frac{i}{l^{2}} \stackrel{\text { cut }}{\longrightarrow} 2 \pi \delta_{+}\left(l^{2}\right)
$$

such that the loop quantities are factorized as product of simpler on-shell building blocks. This cut process captures physical singularities of kinematic functions, and one can reconstruct the analytic function via the information given by its singularities.

The general strategy of performing full-color non-planar unitarity is discussed in section 4.1, then we provide concrete examples in section 4.2 to illustrate the strategy. The results of examples will also be used in the construction of full form factor results in section 5. Some further details and features about (non-planar) unitarity are also provided in appendix B.

\subsection{General strategy}

From the discussion in the previous section, the full-color form factor takes the color decomposition form as

$$
\boldsymbol{F}^{(\ell)}=\sum_{i} \mathcal{C}_{i}\left[\mathcal{F}^{(\ell)}\right]_{\mathcal{C}_{i}}
$$

where $\mathcal{C}_{i}$ are the color factors, and the color-stripped kinematic coefficients are

$$
\left[\mathcal{F}^{(\ell)}\right]_{\mathcal{C}_{i}}=\sum(\text { momentum integrals })
$$

which are what we want to compute using unitarity cut method.

It should be helpful to first review the planar unitarity. The planar form factors, denoted as $\left[\mathcal{F}^{(\ell)}\right]_{\mathrm{PL}}$, satisfy the following planar cut: ${ }^{6}$

$$
\left.\left[\mathcal{F}^{(\ell)}\right]_{\mathrm{PL}}\right|_{\text {cut }}=\int \mathrm{dPS}_{\left\{l_{a}^{2}\right\}} \text { (color-ordered tree products), }
$$

where the phase space measure is understood as $\operatorname{dPS}_{\left\{l_{a}^{2}\right\}}=\prod_{a} d^{D} l_{a} \delta_{+}\left(l_{a}^{2}\right)$. The planar unitarity is simple in two aspects: (1) the tree blocks are simple color-ordered quantities, and (2) the ordering within each blocks and the connection between blocks are completely fixed by planar topology.

\footnotetext{
${ }^{6}$ We consider only tree building blocks here, but sub-loop building blocks are also possible.
} 
Planar form factors can be regarded as special (planar) components of full-color form factors, or in other words, they are the kinematic components associated to planar color factors $\mathcal{T}_{\mathrm{PL}}$ that are leading in the large $N_{c}$ limit. The choosing of the tree blocks is determined by the fact that the product of their trace color factors $\mathcal{T}_{\text {tree-block }}$ contribute the planar color factor:

$$
\prod \mathcal{T}_{\text {tree-block }}=\mathcal{T}_{\mathrm{PL}}+\left(\text { subleading terms in } N_{c}\right) .
$$

This understanding allows us to generalize unitarity cut to kinematic component $\left.{ }^{\left[\mathcal{F}^{(\ell)}\right.}\right]_{\mathcal{C}_{i}}$ with a general color factor $\mathcal{C}_{i}$. Let us first consider $\mathcal{C}_{i}=\mathcal{T}_{i}$ (the trace basis), and we will comment on the cases with other basis shortly. For a generic color factor $\mathcal{T}_{i}$ (which can be multi-trace for example), one can apply unitarity in a similar way as

$$
\left.\left[\mathcal{F}^{(\ell)}\right]_{\mathcal{T}_{i}}\right|_{\text {cut }}=c_{i} \int \mathrm{dPS}_{\left\{l_{a}^{2}\right\}}(\text { color-ordered tree products }),
$$

where the tree products are chosen such that

$$
\prod \mathcal{T}_{\text {tree-block }}=c_{i} \mathcal{T}_{i}+\left(\text { non- } \mathcal{T}_{i} \text { factors }\right),
$$

and $c_{i}$ is a possible numerical factor. Here it is important that tree building blocks are still color-ordered, thus taking advantage of the simplicity of color-ordered quantities. The r.h.s. of (4.6) are specified by the ordering of each tree blocks. We define each such configuration as a cut channel.

A single cut channel typically only probes partial physical singularities of the integrand, therefore, to determine the full kinematic integrand of $\left[\mathcal{F}^{(\ell)}\right]_{\mathcal{T}_{i}}$, one needs to consider a spanning set of cut channels having color factor $\mathcal{T}_{i}$. For planar form factors, usually only a small number of cut channels are needed which can be easily determined by planarity. For the full-color case, a more careful analysis is needed based on (4.6). Different ways of sewing tree blocks as well as different planar ordering of each tree block should be taken into account. This will be explained with explicit examples in section 4.2. In appendix B we also describe some systematic way of classifying cut channels and also discuss some further features of nonplanar cuts.

Finally, to obtain the full form factor, one needs to consider all color factors in the basis. For the trace basis, it seems that every single $\left[\mathcal{F}^{(\ell)}\right]_{\mathcal{T}_{i}}$ requires an independent calculation. However, changing other color basis can reduce the amount of calculation. Thus, let us now comment on the choice of other color basis. If the color basis $\mathcal{C}_{j}$ of full-color form factor $\boldsymbol{F}^{(\ell)}$ is not trace basis, for example is certain trivalent basis (DDM basis or CK ansatz), such that $\mathcal{C}_{j}=\prod \tilde{f}$, one can expand the $\mathcal{C}_{j}$ in terms of trace basis and perform a basis change. For example, for $\mathcal{C}_{j}=b_{j}^{(i)} \mathcal{T}_{i}+$ (non- $\mathcal{T}_{i}$ factors), the kinematic parts from different basis are related as

$$
\left[\mathcal{F}^{(\ell)}\right]_{\mathcal{T}_{i}}=\sum_{j}\left[\mathcal{F}^{(\ell)}\right]_{\mathcal{C}_{j}} b_{j}^{(i)} .
$$

Thus knowing $\left[\mathcal{F}^{(\ell)}\right]_{\mathcal{T}_{i}}$ will be able determine $\left[\mathcal{F}^{(\ell)}\right]_{\mathcal{C}_{j}}$. Practically it is often convenient to start with trivalent DDM basis, which has relatively small number of color basis and can also manifest certain symmetry structure of the results. 


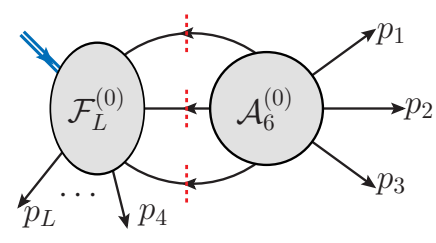

(a)

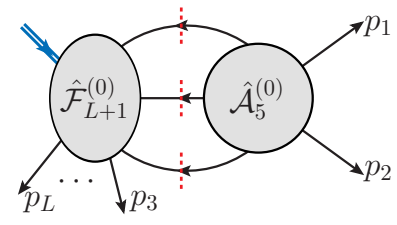

(b)

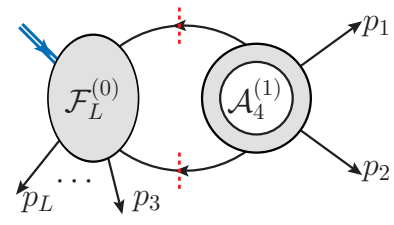

(c)

Figure 5. Planar cut channels at two loops. Without loss of generality, we choose the external lines as $p_{1}, p_{2}, p_{3}$.

The above discussion also applies at the level of density functions $\boldsymbol{I}^{(\ell)}$ defined in section 3. In our computation of two-loop form factors, thanks to the universal structures of density functions, one can select a small subset of $\mathcal{T}_{i}$, such that their kinematic parts are enough to fix all kinematic functions in $\boldsymbol{I}$. This will be one central topic of section 5 . It is worthwhile to mention that, the method of non-planar unitarity also applies to the CK dual ansatz which will be discussed in section 6 .

The above strategy may be summarized in the following graph:

\begin{tabular}{|c|c|c|c|c|c|c|}
\hline $\begin{array}{l}\text { color-ordered } \\
\text { tree blocks }\end{array}$ & $\underset{\text { all channels }}{\stackrel{\text { unitarity-cut }}{\longrightarrow}}$ & $\begin{array}{c}\text { Kinematic } \\
\text { part }\left[\mathcal{F}^{(\ell)}\right]_{\mathcal{T}_{i}}\end{array}$ & $\underset{\text { all } \mathcal{T}_{i}}{\stackrel{\text { collect }}{\longrightarrow}}$ & $\begin{array}{c}\boldsymbol{F}^{(\ell)} \text { in } \\
\text { trace basis }\end{array}$ & $\leftrightarrow$ & $\begin{array}{c}\boldsymbol{F}^{(\ell)} \text { in DDM basis } \\
\text { or CK dual form }\end{array}$ \\
\hline
\end{tabular}

\subsection{Examples for unitarity construction}

We now apply the above strategy to construct some two-loop form factor examples. For simplicity, we choose the operator as half-BPS operators $\operatorname{tr}\left(X^{L}\right)$. These examples will be useful in later full-color form factor construction in section 5 .

Example 1: the planar case. We briefly review the planar limit case. In this case, our target color factor is

$$
\mathcal{T}_{\mathrm{PL}}=N_{c}^{2} \operatorname{tr}\left(a_{1} \cdots a_{L}\right)
$$

To determine the integrand, it is enough to consider three types of cut channels as shown in figure 5.

We start from $s_{123}$ triple-cut (TC) with planar ordering in figure $5(\mathrm{a})$. The product of two trace factors from tree building blocks gives

$$
\operatorname{tr}_{\mathcal{A}}\left(\check{a}_{l_{3}} \check{a}_{l_{2}} \check{a}_{l_{1}} a_{1} a_{2} a_{3}\right) \times \operatorname{tr}_{\mathcal{F}}\left(a_{l_{1}} a_{l_{2}} a_{l_{3}} a_{4} \cdots a_{L}\right)=1 \times \mathcal{T}_{\mathrm{PL}}+\left(\text { subleading terms in } N_{c}\right)
$$

which indeed contains the planar color factor. The kinematic part under this cut is ${ }^{7}$

$$
\begin{aligned}
\left.\mathcal{F}_{\mathrm{PL}}^{(2)}\left(p_{1}, p_{2}, p_{3}, \ldots\right)\right|_{s_{123}-\mathrm{TC}} & =\int \mathrm{dPS}_{3, l} \mathcal{F}_{L}^{(0)}\left(-l_{1},-l_{2},-l_{3}, p_{4}, \ldots, p_{L}\right) \mathcal{A}_{6}^{(0)}\left(l_{3}, l_{2}, l_{1}, p_{1}, p_{2}, p_{3}\right) \\
& ={ }_{p_{3}}
\end{aligned}
$$

\footnotetext{
${ }^{7}$ Terms proportional to $l_{i}^{2}$ are possible contributions which can not be fixed by this cut. Other cuts can detect them so they are contained in "terms fixed by other channels" in (4.13).
} 
where the six-scalar tree amplitude expression (given in appendix $\mathrm{C}$ ) and expression for the tree form factor (here $\mathcal{F}_{L}^{(0)}=1$ ) are used. In the second line of (4.11), the tree product is reorganized as three cut integrals (see [14] for more details). Here for convenience, we introduce in (4.11) a graphic notation to use colored dots to represent numerators: two dots with the same color on lines with momenta $q_{i}$ and $q_{j}$ means $2\left(q_{i} \cdot q_{j}\right)$. For example, the numerators for three integrals in (4.11) are respectively:

$$
4\left(p_{1} \cdot p_{2}\right)\left[p_{3} \cdot\left(l_{1}+p_{1}+p_{2}\right)\right], \quad 4\left(p_{2} \cdot p_{3}\right)\left[p_{1} \cdot\left(l_{3}+p_{2}+p_{3}\right)\right], \quad 2\left(p_{1} \cdot p_{3}\right) .
$$

It should be obvious that $s_{i(i+1)(i+2)}$ triple cuts contribute similar terms as (4.13) by changing $\left\{p_{1}, p_{2}, p_{3}\right\}$ to $\left\{p_{i}, p_{i+1}, p_{i+2}\right\}$. Removing the cuts, the form factor can be expressed as

$$
\begin{aligned}
\mathcal{F}_{\mathrm{PL}}^{(2)}\left(p_{1}, p_{2}, p_{3}, \ldots\right)= & \\
& + \text { (terms fixed by other channels) }
\end{aligned}
$$

Next we consider the $s_{12}$ triple-cut (TC) channels in figure 5(b). As a check, the product of tree color factors gives

$$
\operatorname{tr}_{\mathcal{A}}\left(\check{a}_{l_{3}} \check{a}_{l_{2}} \check{a}_{l_{1}} a_{1} a_{2}\right) \times \operatorname{tr}_{\mathcal{F}}\left(a_{l_{1}} a_{l_{2}} a_{l_{3}} a_{3} \cdots a_{L}\right)=1 \times \mathcal{T}_{\mathrm{PL}}+\left(\text { subleading terms in } N_{c}\right) .
$$

The cut of the kinematic part is:

$$
\begin{aligned}
\mathcal{F}_{\mathrm{PL}}^{(2)} & \left.\left(p_{1}, p_{2}, p_{3}, \ldots, p_{L}\right)\right|_{s_{12} \text {-TC }} \\
\quad & =\int \mathrm{dPS}_{3, l} \hat{\mathcal{F}}_{L+1}^{(0)}\left(-l_{1},-l_{2},-l_{3}, p_{3}, \ldots, p_{L}\right) \hat{\mathcal{A}}_{5}^{(0)}\left(l_{3}, l_{2}, l_{1}, p_{1}, p_{2}\right) .
\end{aligned}
$$

The expressions of five-point tree amplitudes and next-to-minimal form factors are reviewed in section 2.2, and "hat" in (4.15) remind that one should take super form factor/amplitudes or equivalently, summing over all possible internal state configurations. Without going into details, we point out that in this channel only integrals with external momenta $\left\{p_{L}, p_{1}, p_{2}, p_{3}\right\}$ are possibly probed, because the tree blocks only contain these momenta. Given the knowledge from previous $s_{123}$-TC (and similar $s_{L 12}$-TC), the new contribution from this cut reads:

$$
\begin{aligned}
\mathcal{F}_{\mathrm{PL}}^{(2)}\left(p_{1}, p_{2}, p_{3}, \ldots, p_{L}\right)= & =\left(\text { terms from } s_{123^{-}} \text {and } s_{L 12^{-}} \mathrm{TC}\right) \\
& +(\text { terms fixed by other channels }) .
\end{aligned}
$$

Finally, one can check that the $s_{12}$ double-cut in figure $5(\mathrm{c})$ does not give any new contribution. Thus the kinematic function in (4.16) (plus their permutations) satisfies all required cut channels and determines the complete planar form factor results. 


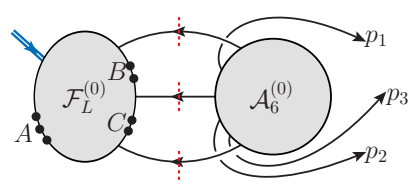

(a) $s_{123}$ triple-cut.

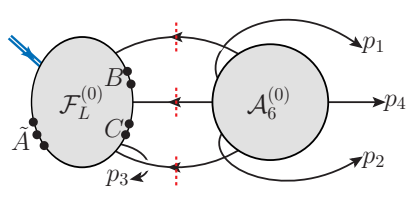

(b) $s_{124}$ triple-cut.

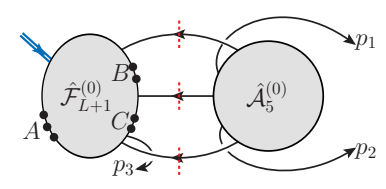

(c) $s_{12}$ triple-cut.

Figure 6. Selected non-planar cut channels at two loops for $\mathcal{F}_{\mathrm{NP}}^{(2)}$. These cuts serves to calculate kinematic dependence of $p_{1}, p_{2}, p_{3}, p_{4}$ for $\mathcal{F}_{\mathrm{NP}}^{(2)}$.

The full planar form factor $\mathcal{F}_{\mathrm{PL}}^{(2)}\left(p_{1}, p_{2}, p_{3}, \ldots, p_{L}\right)$ can be written in a compact form as

$$
\mathcal{F}_{\mathrm{PL}}^{(2)}\left(p_{1}, p_{2}, p_{3}, \ldots, p_{L}\right)=\sum_{i=1}^{L} \widetilde{\mathcal{F}}_{\mathrm{PL}}^{(2)}\left(p_{i}, p_{i+1}, p_{i+2}\right),
$$

where we define a density function (specified by a tilde):

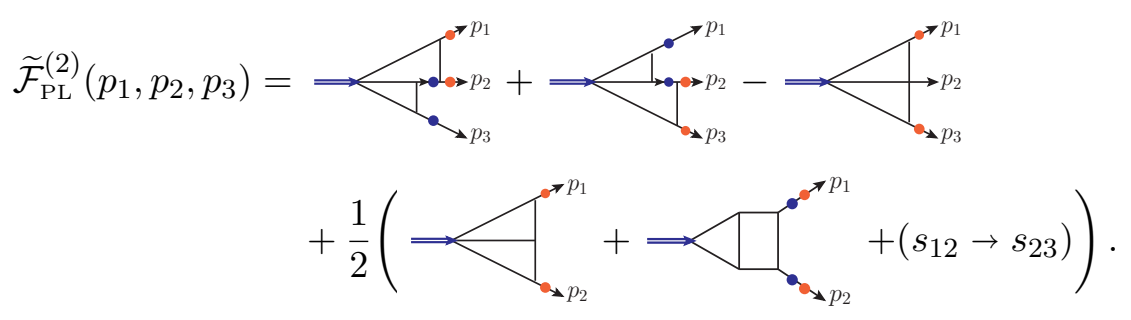

Other density functions are related to it as:

$$
\widetilde{\mathcal{F}}_{\mathrm{PL}}^{(2)}\left(p_{i}, p_{i+1}, p_{i+2}\right)=\left.\widetilde{\mathcal{F}}_{\mathrm{PL}}^{(2)}\left(p_{1}, p_{2}, p_{3}\right)\right|_{\{1 \rightarrow i, 2 \rightarrow(i+1), 3 \rightarrow(i+2)\}} .
$$

Note that the $1 / 2$ factor in (4.18) shows that we have evenly distributed the range- 2 integrals to two density functions.

Example 2: a non-planar case. Next we consider a non-trivial non-planar example with a triple trace color factor as

$$
\mathcal{T}_{\mathrm{NP}}=\operatorname{tr}\left(B a_{1}\right) \operatorname{tr}\left(C a_{3} a_{2}\right) \operatorname{tr}(A)
$$

where we assume $A, B, C$ are all non-empty and assign $A=\left\{a_{4}, \ldots, a_{8}\right\}, B=$ $\left\{a_{7}, \ldots, a_{6}\right\}, C=\left\{a_{5}, \ldots\right\}$, representing other sets of color indices in the traces. As in the previous planar case, we will mainly focus on the cut channels that involve external momenta $\left\{p_{1}, p_{2}, p_{3}\right\}$.

Following the strategy in section 4.1, we need to identify the cut channels that contribute to the color factor $\mathcal{T}_{\text {NP }}$. Let us first consider the $s_{123}$ triple-cut. The allowed configurations are shown in figure $6(\mathrm{a})$. The product of tree factors gives the required color factor:

$$
\operatorname{tr}_{\mathcal{A}}\left(\check{a}_{l_{3}} a_{3} a_{2} \check{a}_{l_{2}} a_{1} \check{a}_{l_{1}}\right) \times \operatorname{tr}_{\mathcal{F}}\left(A a_{l_{1}} B a_{l_{2}} C a_{l_{3}}\right)=1 \times \mathcal{T}_{\mathrm{NP}}+\cdots
$$


where '...' represents other color factors. The kinematic part under this cut is:

$$
\left.\mathcal{F}_{\mathrm{NP}}^{(2)}\left(p_{1}, p_{2}, p_{3}, \ldots\right)\right|_{s_{123}-\mathrm{TC}}=\int \mathrm{dPS}_{3, l} \mathcal{F}_{L}^{(0)}\left(A,-l_{1}, B,-l_{2}, C,-l_{3}\right) \mathcal{A}_{6}^{(0)}\left(l_{3}, p_{3}, p_{2}, l_{2}, p_{1}, l_{1}\right) .
$$

Plugging in the expressions for the tree form factor (here $\mathcal{F}_{L}^{(0)}=1$ ) and the six-scalar tree amplitude $\mathcal{A}^{(0)}\left(l_{3}^{\bar{X}}, p_{3}^{X}, p_{2}^{X}, l_{2}^{\bar{X}}, p_{1}^{X}, l_{1}^{\bar{X}}\right)$ (see appendix C), and after removing the cut, one has

$$
\mathcal{F}_{\mathrm{NP}}^{(2)}\left(p_{1}, p_{2}, p_{3}, \ldots\right)=-\mathcal{K}_{\mathrm{NP}}(123)+(\text { terms determined by other channels }),
$$

where

$$
\begin{aligned}
& \mathcal{K}_{\mathrm{NP}}(123)
\end{aligned}
$$

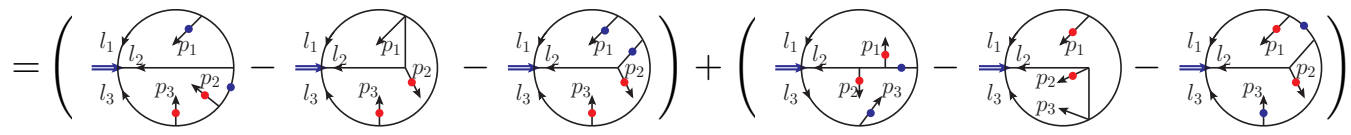

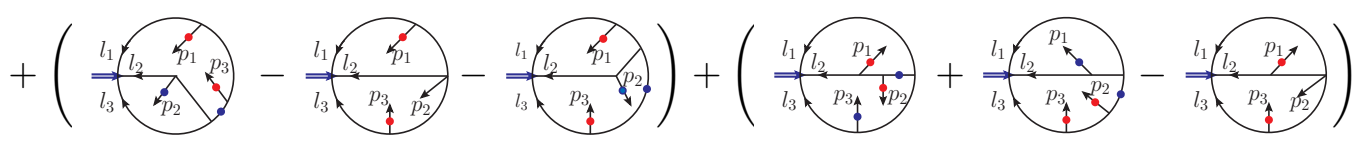

$$
\begin{aligned}
& -\left(\stackrel{l_{3}+\frac{l_{2}}{l_{p_{2}}}}{l}\right.
\end{aligned}
$$

The above expression is straightforward to obtain since the r.h.s. expression is in a oneto-one correspondence with the six-scalar amplitude expression when cutting $l_{i}$ legs. Note that the two graphs underlined cancel between each other, which are topologically actually forbidden regarding the ordering from $\mathcal{A}_{6}^{(0)}\left(l_{3}, p_{3}, p_{2}, l_{2}, p_{1}, l_{1}\right)$. They are purposely inserted and we arrange $\mathcal{F}_{\mathrm{NP}}^{(2)}\left(p_{1}, p_{2}, p_{3}, \ldots\right)$ into this form because it will be helpful for the discussion in section 5.2.

Next we consider a $s_{12}$ triple-cut (TC) channel, and our choice of ordering for blocks in this channel as shown in figure 6(c): the $p_{3}$ leg in figure 6(a) moves to the lower side of $C$. One can check that the color factor is correct:

$$
\operatorname{tr}_{\mathcal{A}}\left(\check{a}_{l_{3}} \check{a}_{l_{2}} a_{2} a_{1} \check{a}_{l_{1}}\right) \times \operatorname{tr}_{\mathcal{F}}\left(A a_{l_{1}} B a_{l_{2}} C a_{3} a_{l_{3}}\right)=1 \times \mathcal{T}_{\mathrm{NP}}+\ldots
$$

The kinematic parts under cut $s_{12}$-TC satisfy:

$$
\begin{aligned}
&\left.\mathcal{F}_{\mathrm{NP}}^{(2)}\left(p_{1}, p_{2}, p_{3}, p_{4}, \ldots\right)\right|_{s_{12}-\mathrm{TC}} \\
& \quad=\int \mathrm{dPS}_{3, l} \hat{\mathcal{F}}_{L+1}^{(0)}\left(-l_{1}, B,-l_{2}, C, p_{3},-l_{3}, p_{4}, \tilde{A}\right) \hat{\mathcal{A}}_{5}^{(0)}\left(l_{1}, l_{3}, l_{2}, p_{2}, p_{1}\right),
\end{aligned}
$$

in which the tree five-point amplitude and next-to-minimal form factors are reviewed in section 2.2. And we define $\tilde{A}$ so that $\left\{p_{4}, \tilde{A}\right\}=A$. 
To simplify the discussion, we note that the next-to-minimal form factor $\hat{\mathcal{F}}_{L+1}^{(0)}$ in (4.26) can be split into three (gauge invariant) parts according to the kinematic dependence ${ }^{8}$

$$
\begin{aligned}
\hat{\mathcal{F}}_{L+1}^{(0)} & \left(-l_{1}, B,-l_{2}, C, p_{3},-l_{3}, p_{4}, \tilde{A}\right) \\
& =\hat{\mathcal{F}}_{L+1, \text { dens }}^{(0)}\left(p_{3},-l_{3}, p_{4}\right)+\hat{\mathcal{F}}_{L+1, \text { dens }}^{(0)}\left(p_{5},-l_{2}, p_{6}\right)+\hat{\mathcal{F}}_{L+1 \text {,dens }}^{(0)}\left(p_{7},-l_{1}, p_{8}\right) .
\end{aligned}
$$

Since the three terms within (4.27) are gauge invariant respectively and depend on different external momenta, one can calculate them separately. We thus decompose (4.26) correspondingly as

$$
\begin{aligned}
& \left.\mathcal{F}_{\mathrm{NP}}^{(2)}\left(p_{1}, p_{2}, p_{3}, p_{4}, \ldots\right)\right|_{s_{12}-\mathrm{TC}} \\
& \quad=\int \mathrm{d} \hat{\mathrm{P}} \mathrm{S}_{3, l} \hat{\mathcal{F}}_{L+1, \mathrm{dens}}^{(0)}\left(p_{3},-l_{3}, p_{4}\right) \hat{\mathcal{A}}_{5}^{(0)}\left(l_{1}, l_{3}, l_{2}, p_{2}, p_{1}\right)+\left(\text { terms dependent on } p_{5} \text { to } p_{8}\right) .
\end{aligned}
$$

Let us focus on the first term on the r.h.s. of (4.28). Similar to the planar example, this part also probes terms from $s_{123}$ and $s_{124}$ triple-cut. For example, the cut contribution from the integrand $-\mathcal{K}_{\mathrm{NP}}(123)$ in $(4.24)$ is

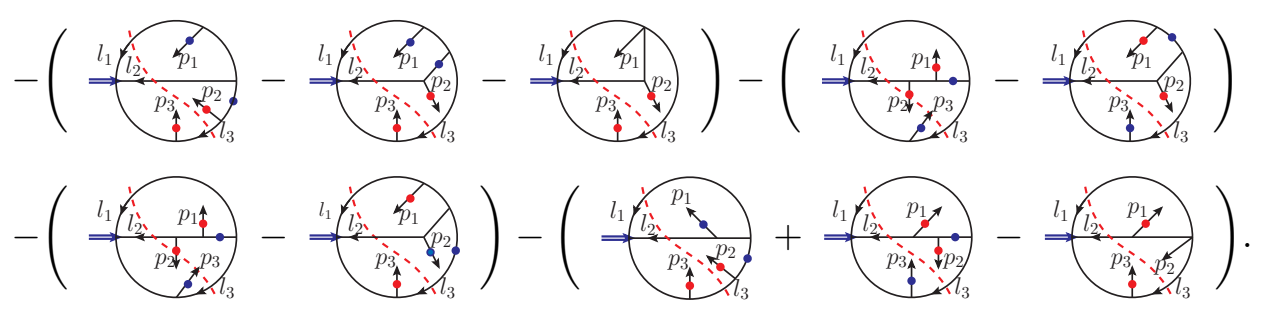

The contribution from $s_{124}$-cut-detectable terms, with such a cut in figure $6(\mathrm{~b})$, is similar and can be obtained from the $s_{123}$-cut result (4.29) using the following relation:

$$
s_{124} \text {-cut-detectable terms }=s_{123} \text {-cut-detectable terms }\left.\right|_{p_{3} \rightarrow p_{4}, l_{1} \leftrightarrow l_{2}, p_{1} \leftrightarrow p_{2}} .
$$

Besides these cut contributions, there appears a new contribution detected by the $s_{12^{-}}$ triple cut:

$$
-\frac{s_{12}}{s_{1 l_{1}} s_{2 l_{2}}}=-\frac{1}{2} \stackrel{p_{1}}{\Rightarrow}
$$

Note that this is a range-2 integral and is obviously vanishing under the $s_{123}$-triple cut. Similar to the calculation of the first term of (4.28), the kinematic dependence on $p_{5}$ to $p_{8}$ can be fixed in a similar way and we will not reproduce the details here.

So far we have considered $s_{12}$ triple-cut channel. To determine the integrand that involves $\left\{p_{1}, p_{2}, p_{3}\right\}$, the remaining cuts to consider are $s_{23}$ triple-cut and $s_{13}$ triple-cut. They can be considered in the same way as for the $s_{12}$ triple-cut, and one finds two similar range-2 functions as (4.31), with kinematic dependence on $s_{13}$ and $s_{23}$ respectively.

\footnotetext{
${ }^{8}$ Note that no fermions can be exchanged between internal lines because of non-adjacency of $l_{i}$ in $\hat{\mathcal{F}}_{L+1}^{(0)}\left(-l_{1}, B,-l_{2}, C, p_{3},-l_{3}, A\right)$.
} 
Including these range-2 contribution to (4.24), one can introduce a density function $\widetilde{\mathcal{F}}_{\mathrm{NP}}^{(2)}\left(p_{1}, p_{2}, p_{3}\right)$, similar to planar density in $(4.18)$, as

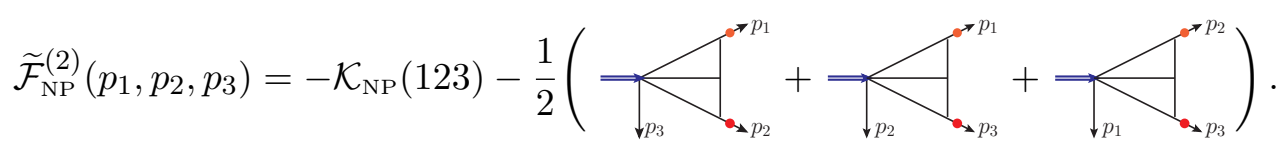

so that the full form factor can be given by summing all density functions as

$$
\mathcal{F}_{\mathrm{NP}}^{(2)}=\sum_{i<j<k} \widetilde{\mathcal{F}}_{\mathrm{NP}}^{(2)}\left(p_{i}, p_{j}, p_{k}\right)
$$

The difference, comparing to the planar case, is that $\widetilde{\mathcal{F}}_{\mathrm{NP}}^{(2)}\left(p_{i}, p_{j}, p_{k}\right)$ is no longer a universal function as (4.19):

$$
\widetilde{\mathcal{F}}_{\mathrm{NP}}^{(2)}\left(p_{i}, p_{j}, p_{k}\right) \neq\left.\widetilde{\mathcal{F}}_{\mathrm{NP}}^{(2)}\left(p_{1}, p_{2}, p_{3}\right)\right|_{\{1 \rightarrow i, 2 \rightarrow j, 3 \rightarrow k\}},
$$

so in principle, we need to calculate every $(i, j, k)$ separately, following the same procedure from (4.20) to (4.32). However, as will be shown in next section, with a proper analysis of color basis, knowing $\widetilde{\mathcal{F}}_{\mathrm{NP}}^{(2)}\left(p_{1}, p_{2}, p_{3}\right)$ is actually enough. Finally, we simplify the representation of the integrals by flatten the figures in a more convenient form as:

$$
\widetilde{\mathcal{F}}_{\mathrm{NP}}^{(2)}\left(p_{1}, p_{2}, p_{3}\right)
$$

\section{Full-color two-loop form factors}

Following section 4, we now turn to the construction of the complete full-color form factors using the unitarity data obtained in the previous section. Before going into the details, let us first clarify the general structure of two-loop form factors. The two-loop form factors have the following schematic structure (a more precise form can be obtained following (3.8) which is not important for later discussions):

$$
\boldsymbol{F}^{(2)}=\boldsymbol{I}^{(2)} \boldsymbol{F}^{(0)}=\left(\sum_{i<j<k} \boldsymbol{I}_{i j k}^{(2)}+\sum_{i<j} \boldsymbol{I}_{i j}^{(2)}+\sum_{i j \neq k l} \boldsymbol{I}_{i j}^{(1)} \boldsymbol{I}_{k l}^{(1)}\right) \boldsymbol{F}^{(0)} .
$$

The last part $\sum_{i j \neq k l} \boldsymbol{I}_{i j}^{(1)} \boldsymbol{I}_{k l}^{(1)}$ corresponds to the product of two decoupled one-loop corrections, which is trivial and thus ignored in the following discussion. The first two parts 


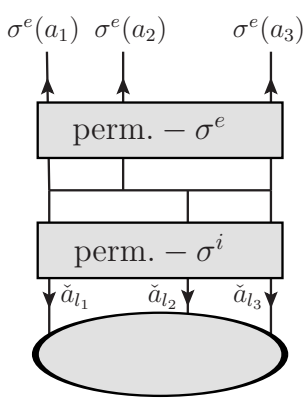

(a) Cubic Graph: $\check{\mathcal{D}}_{\Delta_{1}}$

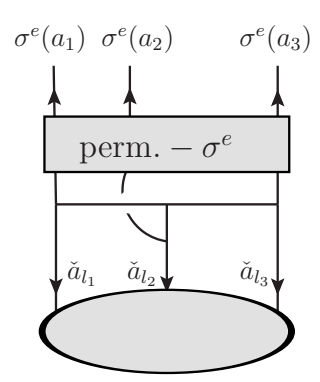

(b) Cubic Graph: $\check{\mathcal{D}}_{\Delta_{2}}$

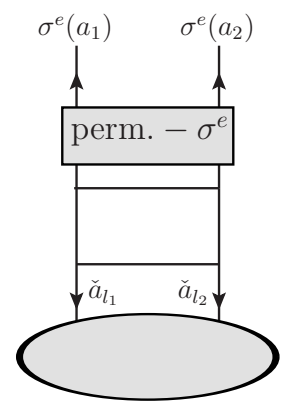

(c) Cubic Graph: $\check{\mathcal{D}}_{\Delta_{3}}$

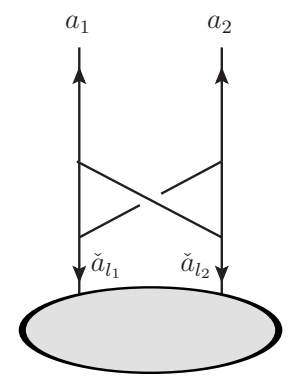

(d) Cubic Graph: $\check{\mathcal{D}}_{\Delta_{4}}$

Figure 7. Cubic Graphs for minimal two-loop correction density.

are intrinsic two-loop corrections, and we separate them as range- 3 and range- 2 densities respectively. To calculate full form factors, one only needs to compute the following density

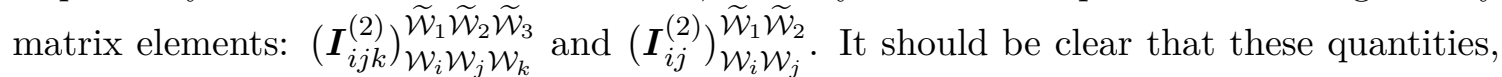
as function of momenta, depend also on the transition of states (i.e. fields).

Below we will first consider the color decomposition for $\boldsymbol{I}^{(2)}$ which will help to identify a set of kinematic functions that are enough to determine $\boldsymbol{I}^{(2)}$. As will be shown in section 5.1, by means of properly selecting $\mathcal{T}_{i}$ and $\left[\mathcal{F}^{(2)}\right]_{\mathcal{T}_{i}}$, these functions can be determined by a small set of equations. Then, we will compute two classes of form factors: (1) the half-BPS form factors with $\operatorname{tr}\left(X^{L}\right)$ in section 5.2; (2) the form factor with non-BPS operators in the $\mathrm{SU}(2)$ sector in section 5.3 .

\subsection{Color decomposition of two-loop form factors}

In this subsection we consider the color decomposition for the density functions as in (3.9):

$$
\begin{aligned}
\left(\boldsymbol{I}^{(2)}\right)_{\mathcal{W}_{i} \mathcal{W}_{j} \mathcal{W}_{k}}^{\widetilde{\mathcal{W}}_{1} \widetilde{\mathcal{W}}_{3} \widetilde{\mathcal{W}}_{3}}(123)=\sum_{\alpha} \check{\mathcal{C}}_{\alpha}\left[\left(\mathcal{I}^{(2)}\right)_{\mathcal{W}_{i} \mathcal{W}_{j} \mathcal{W}_{k}}^{\widetilde{\mathcal{W}}_{1} \widetilde{\mathcal{W}}_{2} \widetilde{\mathcal{W}}_{3}}(123)\right]_{\check{\mathcal{C}}_{\alpha}}, \\
\left(\boldsymbol{I}^{(2)}\right)_{\mathcal{W}_{i} \mathcal{W}_{j}}^{\widetilde{\mathcal{W}}_{1} \widetilde{\mathcal{W}}_{2}}(12)=\sum_{\beta} \check{\mathcal{C}}_{\beta}\left[\left(\mathcal{I}^{(2)}\right)_{\mathcal{W}_{i} \mathcal{W}_{j}}^{\widetilde{\mathcal{W}}_{1} \widetilde{\mathcal{W}}_{2}}(12)\right]_{\check{\mathcal{C}}_{\beta}} .
\end{aligned}
$$

Here we will explicitly construct the DDM basis as $\check{\mathcal{C}}_{\alpha}$, and discuss how to fix the kinematics parts $\mathcal{I}$.

As a remark on notations, without loss of generality we choose the external momenta to be $\left\{p_{1}, p_{2}, p_{3}\right\}$ and set labeling of $\boldsymbol{I}_{i j k}^{(2)}$ as $\boldsymbol{I}_{i j k}^{(2)}(123)$ and $\boldsymbol{I}_{i j}^{(2)}$ as $\boldsymbol{I}_{i j}^{(2)}(12)$. Meanwhile, the color indices of external states are $a_{1}, a_{2}, a_{3}$. When there is no ambiguity, the momenta label (123) and (12) can be omitted for simplicity.

DDM basis. Consider first the range-3 color basis. The trivalent topologies can be obtained by sewing six-point tree-level topologies to minimal form factors. One can choose two independent configurations whose diagrammatical representations are given in figure $7(\mathrm{a})$ and 7(b), respectively. They correspond to the following two sets of color structures:

$$
\begin{aligned}
& \text { Set }_{1}: \quad\left\{\check{\mathcal{D}}_{\Delta_{1}}\left(\sigma^{e}, \sigma^{i}\right)\right\}=\left\{\sigma^{i} \sigma^{e} \cdot f^{\check{a}_{l_{1}} a_{1} \mathrm{x}_{1}} f^{\mathrm{x}_{1} a_{2} \mathrm{x}_{2}} f^{\mathrm{x}_{2} \mathrm{x}_{3} \check{a}_{l_{2}}} f^{\mathrm{x}_{3} a_{3} \check{a}_{l_{3}}}\right\} \text {, } \\
& \text { Set }_{2}: \quad\left\{\check{\mathcal{D}}_{\Delta_{2}}\left(\sigma^{e}\right)\right\}=\left\{\sigma^{e} \cdot f^{\check{a}_{l_{1}} a_{1} \mathrm{x}_{1}} f^{\check{a}_{l_{2}} a_{2} \mathrm{x}_{2}} f^{\check{a}_{l_{3}} a_{3} \mathrm{x}_{3}} f^{\mathrm{x}_{1} \mathrm{x}_{2} \mathrm{x}_{3}}\right\} \text {, }
\end{aligned}
$$


where the permutations $\sigma_{3}^{e} \in S_{3}$ and they act on color indices of external states $\left\{a_{1}, a_{2}, a_{3}\right\}$, while $\sigma_{3}^{i} \in \mathbb{Z}_{3}$ (cyclic permutation) act on internal color variation $\left\{\check{a}_{l_{1}}, \check{a}_{l_{2}}, \check{a}_{l_{3}}\right\}$ (acting on three sites $\{i, j, k\}$ in the operator). As a check, the number of elements of the above range- 3 basis is $3 ! \times 3+3 !=24$, which is the same as the dimension of DDM basis for six-point amplitude $\boldsymbol{A}_{6}^{(0)}$.

The range-2 cubic graphs can be understood as sewing a one-loop four-point cubic graph on minimal form factors. There are also two independent configurations as shown in figure $7(\mathrm{c})$ and $7(\mathrm{~d})$. Thus, range-2 color basis for minimal two-loop correction density can be given as

$$
\begin{array}{ll}
\text { Set }_{3}: & \left\{\check{\mathcal{D}}_{\Delta_{3}}\left(\sigma^{e}\right)\right\}=\left\{\sigma^{e} \cdot f^{\mathrm{x}_{1} \check{a}_{l_{2}} \mathrm{x}_{2}} f^{\mathrm{x}_{2} \check{a}_{l_{1}} \mathrm{x}_{3}} f^{\mathrm{x}_{3} a_{1} \mathrm{x}_{4}} f^{\mathrm{x}_{4} a_{2} \mathrm{x}_{1}}\right\} \\
\text { Set }_{4}: & \left\{\check{\mathcal{D}}_{\Delta_{4}}(\mathbf{1})\right\}=\left\{f^{\mathrm{x}_{1} \check{a}_{l_{2} \mathrm{x}_{2}}} f^{\mathrm{x}_{2} a_{2} \mathrm{x}_{3}} f^{\mathrm{x}_{3} a_{1} \mathrm{x}_{4}} f^{\mathrm{x}_{4} \check{a}_{l_{1}} \mathrm{x}_{1}}\right\}
\end{array}
$$

where the permutations $\sigma_{2}^{e} \in S_{2}$ and they act on color indices of external states $\left\{a_{1}, a_{2}\right\}$.

For the convenience of notation, we will use an explicit set of color factors $\check{\mathcal{D}}_{i}$ to represent the elements in $\check{\mathcal{D}}_{\Delta_{a}}$ : (1) $\check{\mathcal{D}}_{i}, i=1, \ldots, 18$, for 18 elements $\check{\mathcal{D}}_{\Delta_{1}}$ in $(5.4)$, (2) $\check{\mathcal{D}}_{j}$, $j=19, \ldots, 24$, for 6 elements $\check{\mathcal{D}}_{\Delta_{2}}$ in $(5.5),(3) \check{\mathcal{D}}_{25}, \check{\mathcal{D}}_{26}$ for 2 elements $\check{\mathcal{D}}_{\Delta_{3}}$ in (5.6), and (4) $\check{\mathcal{D}}_{27}$ for the single element $\check{\mathcal{D}}_{\Delta_{4}}$ in (5.7). Explicit form of $\check{\mathcal{D}}_{i}$ are listed in appendix A. Four of the them will appear frequently in the following discussion, and we give out the expressions:

$$
\check{\mathcal{D}}_{1}=\check{\mathcal{D}}_{\Delta_{1}}(\mathbf{1}, \mathbf{1}), \quad \check{\mathcal{D}}_{19}=\check{\mathcal{D}}_{\Delta_{2}}(\mathbf{1}), \quad \check{\mathcal{D}}_{25}=\check{\mathcal{D}}_{\Delta_{3}}(\mathbf{1}), \quad \check{\mathcal{D}}_{27}=\check{\mathcal{D}}_{\Delta_{4}}(\mathbf{1})
$$

The permutational relations for the color factors imply that the kinematic parts also satisfy certain permutational relations:

$$
\begin{aligned}
& {\left[\left(\mathcal{I}^{(2)}\right)_{\mathcal{W}_{i} \mathcal{W}_{j} \mathcal{W}_{k} \widetilde{\mathcal{W}}_{2} \widetilde{\mathcal{W}}_{3}}(123)\right]_{\Delta_{i}\left(\sigma^{e}, \sigma^{i}\right)}=\left[\left(\mathcal{I}^{(2)}\right)_{\sigma^{i}\left(\mathcal{W}_{i} \mathcal{W}_{j} \mathcal{W}_{k}\right)}^{\sigma^{e}\left(\widetilde{\widetilde{N}}_{1} \widetilde{\widetilde{W}} \widetilde{\mathcal{W}}_{3}\right)}\left(\sigma^{e}(123)\right)\right]_{\Delta_{i}(\mathbf{1}, \mathbf{1})},} \\
& {\left[\left(\mathcal{I}^{(2)}\right)_{\mathcal{W}_{i} \mathcal{W}_{j}}^{\widetilde{\mathcal{W}}_{1} \widetilde{\mathcal{W}}_{2}}(12)\right]_{\Delta_{i}\left(\sigma^{e}\right)}=\left[\left(\mathcal{I}^{(2)}\right)_{\left(\mathcal{W}_{i} \mathcal{W}_{j}\right)}^{\sigma^{e}\left(\widetilde{\mathcal{W}}_{2} \widetilde{\widetilde{N}}_{2}\right)}\left(\sigma^{e}(12)\right)\right]_{\Delta_{i}(\mathbf{1})} .}
\end{aligned}
$$

This observation implies that one can focus on the following correction functions:

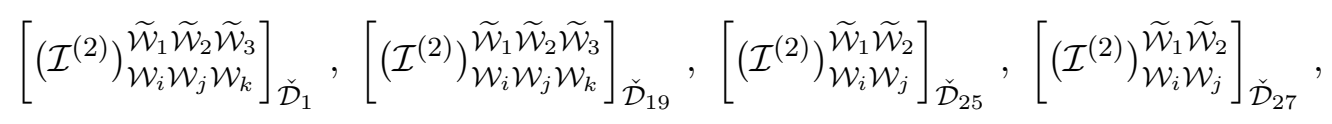

taking into account all possible field configurations of $\mathcal{W}$ and $\widetilde{\mathcal{W}}$.

Equations for solving correction functions. As discussed in section 4.1, to apply unitarity, it is convenient to relate the above trivalent basis to trace basis. In the following content, we discuss their relations and select proper $\mathcal{T}_{i}$ and corresponding $\left[\mathcal{F}^{(2)}\right]_{\mathcal{T}_{i}}$ with which one can determine the four types of correction functions defined in (5.11).

The first relation comes from planar form factor with color factor

$$
\mathcal{T}_{t_{1}} \equiv \mathcal{T}_{\mathrm{PL}}=N_{c}^{2} \operatorname{tr}\left(a_{1} \cdots a_{L}\right)
$$


From the trivalent topologies in table 7 , it is not hard to see that only $\check{\mathcal{D}}_{1}$ and $\check{\mathcal{D}}_{25}$ contribute to the planar topology. Indeed, one can obtain

$$
\begin{aligned}
{\left[\mathcal{F}^{(2)}\right]_{\mathcal{T}_{t_{1}}} } & =\left.\left(\sum_{i<j<k} \boldsymbol{I}_{i j k}^{(2)}+\sum_{i<j} \boldsymbol{I}_{i j}^{(2)}\right) \boldsymbol{F}^{(0)}\right|_{\mathcal{T}_{t_{1}}} \\
& =\left(\left[\mathcal{I}^{(2)}(123)\right]_{\check{\mathcal{D}}_{1}}+\left[\mathcal{I}^{(2)}(12)\right]_{\check{\mathcal{D}}_{25}}+\left[\mathcal{I}^{(2)}(23)\right]_{\check{\mathcal{D}}_{25}}+\cdots\right) \mathcal{F}^{(0)}\left(p_{1}, \cdots, p_{L}\right)
\end{aligned}
$$

which involves only the first and third type of correction functions in (5.11). Let us explain (5.13) (and similar equations below) in more detail:

- For simplicity, we temporarily hide the field index $\mathcal{W}_{i}$ and $\widetilde{\mathcal{W}}_{i}$ and the '...' in the bracket represents similar density functions depending on other momenta.

- To reach the expansion of (5.13), a careful analysis on color structures is required. Concretely, only $\check{\mathcal{D}}_{1}$ and $\check{\mathcal{D}}_{25}$ acting on adjacent sites can give non-vanishing contribution to $\mathcal{T}_{t_{1}}$ :

$$
\begin{aligned}
\check{\mathcal{D}}_{1} \cdot \operatorname{tr}\left(l_{1} l_{2} l_{3} a_{4} \cdots a_{L}\right) & =1 \times \mathcal{T}_{t_{1}}+\cdots \\
\check{\mathcal{D}}_{25} \cdot \operatorname{tr}\left(l_{1} l_{2} a_{3} a_{4} \cdots a_{L}\right) & =1 \times \mathcal{T}_{t_{1}}+\cdots
\end{aligned}
$$

As a result,

$$
\begin{aligned}
\boldsymbol{I}_{123}^{(2)}(123) & \left.\cdot\left(\operatorname{tr}\left(a_{l_{1}} a_{l_{2}} a_{l_{3}} \cdots a_{L}\right) \mathcal{F}^{(0)}\left(p_{1}, p_{2}, p_{3}, \ldots, p_{L}\right)\right)\right|_{\mathcal{T}_{t_{1}}} \\
& =\left.\check{\mathcal{D}}_{1} \cdot \operatorname{tr}\left(a_{l_{1}} a_{l_{2}} a_{l_{3}} \cdots a_{L}\right)\right|_{\mathcal{T}_{t_{1}}} \times\left[\mathcal{I}^{(2)}\right]_{\check{\mathcal{D}}_{1}} \cdot \mathcal{F}^{(0)}\left(p_{1}, p_{2}, p_{3}, \ldots, p_{L}\right) \\
& =\left[\mathcal{I}^{(2)}(123)\right]_{\check{\mathcal{D}}_{1}} \times \mathcal{F}^{(0)}\left(p_{1}, p_{2}, p_{3}, \ldots, p_{L}\right)
\end{aligned}
$$

which explains the corresponding term appearing in (5.13).

- The reason why we only explicitly show the three terms in (5.13) is mainly because they are relevant to the cuts to consider below. Based on the unitarity considerations in the last section, our practical use of equations like (5.13) is to put them under cuts. Hence, to consider only those $\mathcal{I}$ functions involved in certain unitarity channel is sufficient.

The second equation comes from a triple-trace color factor ${ }^{9}$

$$
\mathcal{T}_{t_{2}} \equiv \mathcal{T}_{\mathrm{NP}}=\operatorname{tr}\left(B a_{1}\right) \operatorname{tr}\left(C a_{3} a_{2}\right) \operatorname{tr}(A)
$$

discussed in the second example of section 4.2. This triple-trace color factor is intentionally selected because: (1) range-2 color factors $\check{\mathcal{D}}_{\Delta_{3}}$ and $\check{\mathcal{D}}_{\Delta_{4}}$ do not contribute, one can just focus on range-3 densities consequently; and (2) to split the trace of a tree-level color factor (single-trace) into a triple-trace color factor, the sites on which the loop correction density

\footnotetext{
${ }^{9}$ Note that we focus on single trace operator for simplicity.
} 
acts must be non-adjacent, separated by $A, B, C$ in $\mathcal{T}_{t_{2}}$. More detailed examination shows that only $\check{\mathcal{D}}_{i}$ with $i=11,18,19$ contributes:

$$
\left.\check{\mathcal{D}}_{11} \cdot \operatorname{tr}\left(A l_{1} B l_{2} C l_{3}\right)\right|_{\mathcal{T}_{t_{2}}}=-1,\left.\check{\mathcal{D}}_{18} \cdot \operatorname{tr}\left(A l_{1} B l_{2} C l_{3}\right)\right|_{\mathcal{T}_{t_{2}}}=1,\left.\check{\mathcal{D}}_{19} \cdot \operatorname{tr}\left(A l_{1} B l_{2} C l_{3}\right)\right|_{\mathcal{T}_{t_{2}}}=1,
$$

where explicit expressions of $\check{\mathcal{D}}_{11,18}$ can be found in appendix A. As a result, the kinematic part satisfies the following equation

$$
\left[\mathcal{F}^{(2)}\right]_{\mathcal{T}_{t_{2}}}=\left(\left[\mathcal{I}^{(2)}(123)\right]_{\check{\mathcal{D}}_{11}}-\left[\mathcal{I}^{(2)}(123)\right]_{\check{\mathcal{D}}_{18}}+\left[\mathcal{I}^{(2)}(123)\right]_{\check{\mathcal{D}}_{19}}+\cdots\right) \mathcal{F}^{(0)}\left(A, p_{1}, B, p_{2}, C, p_{3}\right),
$$

where '.. ' in brackets represents functions depending on other momenta. Since $\left[\mathcal{I}^{(2)}\right]_{\check{\mathcal{D}}_{i}}$ with $i=1, \ldots, 18$ can be regarded as known from the previous relation (5.13), the new relation (5.16) can be used to determine $\left[\mathcal{I}^{(2)}\right]_{\check{\mathcal{D}}_{19}}$.

Finally, we need an equation to determine the last term in (5.11). We consider the color factor:

$$
\mathcal{T}_{t_{3}}=N_{\mathrm{c}} \operatorname{tr}\left(a_{1} B\right) \operatorname{tr}\left(a_{2} A\right)
$$

with $A=\left\{a_{4}, \ldots, a_{5}\right\} \equiv\left\{a_{4}, \tilde{A}\right\}$ and $B=\left\{a_{6}, \ldots, a_{3}\right\} \equiv\left\{\tilde{B}, a_{3}\right\}$. A similar analysis can be performed based on the feature of target color factor, and we observe that any range3 density contributing to such color factor must act on sites with two of the three sites adjacent. One find the equation for the kinematic factors as

$$
\begin{aligned}
{\left[\mathcal{F}^{(2)}\right]_{\mathcal{T}_{t_{3}}}=} & \left(\left[\mathcal{I}^{(2)}(12)\right]_{\check{\mathcal{D}}_{27}}+\left[\mathcal{I}^{(2)}(123)\right]_{\check{\mathcal{D}}_{19}}+\left[\mathcal{I}^{(2)}(123)\right]_{\check{\mathcal{D}}_{11}}-\left[\mathcal{I}^{(2)}(123)\right]_{\check{\mathcal{D}}_{18}}\right. \\
& \left.+\left[\mathcal{I}^{(2)}(124)\right]_{\check{\mathcal{D}}_{21}}+\left[\mathcal{I}^{(2)}(124)\right]_{\check{\mathcal{D}}_{5}}-\left[\mathcal{I}^{(2)}(124)\right]_{\check{\mathcal{D}}_{15}}+\cdots\right) \cdot \mathcal{F}^{(0)}\left(p_{1}, A, p_{2}, B\right),
\end{aligned}
$$

which involves the wanted function $\left[\mathcal{I}^{(2)}\right]_{\check{\mathcal{D}}_{27}}$. One can check the color factors are consistent, for example:

$$
\left.\check{\mathcal{D}}_{27} \cdot \operatorname{tr}\left(l_{1} A l_{2} B\right)\right|_{\mathcal{T}_{t_{3}}}=1 .
$$

The three equations (5.13), (5.16), (5.18) provide a minimal set of equations for determining density $\mathcal{I}$ functions, which means that after solving these three equations, we can find a solution satisfying all unitarity cuts for $\boldsymbol{F}$ (expressed via $\boldsymbol{I}$ ). One may notice that there are only three equations while we have four functions to solve. This is due to the fact that there is a degree of freedom in defining the density functions, which is expected as a redistribution property for form factors. The full form factor is certainly independent of such definition. We will also see, at the end of section 6 , an explicit formula to explain how the redistribution works.

\subsection{Two-loop BPS correction}

In this subsection, we apply the above strategy to compute the half-BPS form factors of operators $\operatorname{tr}\left(X^{L}\right)$. Since all the fields are identical, we omit the labeling of fields $\mathcal{W}_{i}$. Discussion here provides details for (5.13)-(5.18). Besides the computation for $\mathcal{F}_{\mathcal{T}_{1,2,3}}$ mentioned in previous subsection, we will also perform another non-trivial check with a different color factor. 


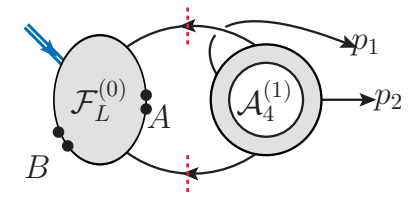

(a) $s_{12}$ double-cut.

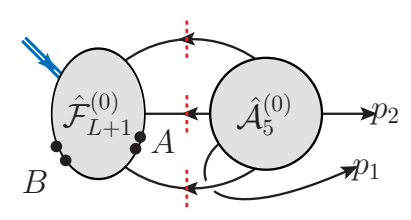

(b) a $s_{12}$ triple-cut.

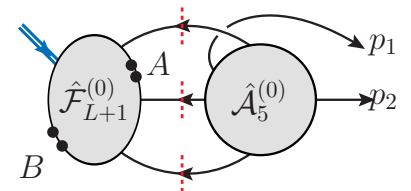

(c) another $s_{12}$ triple-cut.

Figure 8. Selected non-planar cut channels at two loops for $\mathcal{F}_{\mathcal{T}_{t_{3}}}^{(2)}$. These cuts are sufficient to determine $[\mathcal{I}]_{\check{\mathcal{D}}_{27}}^{(2)}$.

Kinematic parts of BPS loop correction in trivalent basis. To begin with, we consider (5.13). This equation involves $\mathcal{I}_{\check{\mathcal{D}}_{1}}$ and $\mathcal{I}_{\check{\mathcal{D}}_{25}}$. Comparing (4.16) with (5.13), a natural choice is

$$
\left[\mathcal{I}^{(2)}(12)\right]_{\check{\mathcal{D}}_{25}}=\Longrightarrow \overbrace{\operatorname{ap}_{p_{2}}}^{p_{1}}
$$

and

$$
\left[\mathcal{I}^{(2)}(123)\right]_{\check{\mathcal{D}}_{1}}=\Longrightarrow
$$

Note that there is some freedom of distributing the range- 2 integrals to the range- 3 result, so the above choice is not unique. As we will discuss in section 7, (5.19)-(5.20) and other results derived later also provide a convenient form for the IR subtraction.

As for the second relation (5.16), one can directly apply (4.35) and (5.16). The form of (4.35) is intentionally arranged such that every row maps to one of the terms in (5.16). Terms with color factor $\check{\mathcal{D}}_{11}$ and $\check{\mathcal{D}}_{18}$ are related to $\check{\mathcal{D}}_{1}$ (via permutation) and correspond to the last two rows in (4.35). For example, $\check{\mathcal{D}}_{11}=\check{\mathcal{D}}_{\Delta_{1}}\left(\left(p_{1}, p_{3}\right),\left(l_{3}, l_{1}, l_{2}\right)\right)$ and

$$
\left[\mathcal{I}^{(2)}\right]_{\check{\mathcal{D}}_{11}}=\left.\left[\mathcal{I}^{(2)}\right]_{\check{\mathcal{D}}_{1}}\right|_{p_{1} \rightarrow p_{2} \rightarrow p_{3}}=\left(\longrightarrow p_{p_{p_{2}}}^{\rightarrow p_{p_{2}}}+\underset{p_{p_{2}}}{p_{p_{3}}}\right) .
$$

Consequently,

$$
\begin{aligned}
{\left[\mathcal{I}^{(2)}(123)\right]_{\check{\mathcal{D}}_{19}}=} & -\left(\underset{p_{2}}{+\operatorname{cyc}\left(p_{1}, p_{2}, p_{3}\right) .}\right.
\end{aligned}
$$

This form apparently satisfy the symmetries as expected.

Finally, we have the third relation (5.18). The cuts we will use to determine part of $\mathcal{F}$ are presented in figure 8. Since our purpose is to fix the range-2 density function, a simple starting point is the two-particle double-cut (DC), as shown in figure 8(a). One can first 
check the color factor:

$$
N_{c} \operatorname{tr}_{\mathcal{A}}\left(\check{a}_{l_{2}} a_{1} \check{a}_{l_{1}} a_{2}\right) \times \operatorname{tr}_{\mathcal{F}}\left(a_{l_{1}} A a_{l_{2}} B\right)=1 \times \mathcal{T}_{t_{3}}+\cdots
$$

The kinematic part under cut is:

$$
\left[\mathcal{F}^{(2)}\right]_{s_{12}-\mathrm{DC}}=\int \mathrm{dPS}_{2, l} \mathcal{F}_{L}^{(0)}\left(-l_{1}, A,-l_{2}, B\right) \mathcal{A}_{4}^{(1)}\left(l_{2}, p_{1}, l_{1}, p_{2}\right),
$$

where it is easy to derived the r.h.s. as

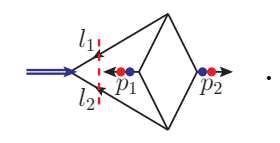

Since no other terms in (5.18) possibly contribute to this cross-ladder integral, it has to come from $\left[\mathcal{I}^{(2)}(12)\right]_{\check{\mathcal{D}}_{27}}$.

Furthermore, we also consider a $s_{12}$ triple-cut (TC) as shown in figure $8(\mathrm{~b})$. The color factor is:

$$
\operatorname{tr}_{\mathcal{A}}\left(\check{a}_{l_{2}} \check{a}_{l_{1}} a_{2} \check{a}_{l_{3}} a_{1}\right) \times \operatorname{tr}_{\mathcal{F}}\left(a_{l_{1}} a_{l_{2}} A a_{l_{3}} B\right)=1 \times \mathcal{T}_{t_{3}}+\cdots,
$$

and the kinematic part under the cut is:

$$
\left.\left[\mathcal{F}^{(2)}\right]_{\mathcal{T}_{t_{3}}}\right|_{s_{12} \text {-TC }}=\int \mathrm{dPS}_{2, l} \hat{\mathcal{F}}_{L+1}^{(0)}\left(-l_{1},-l_{2}, A,-l_{3}, B\right) \hat{\mathcal{A}}_{5}^{(0)}\left(l_{2}, l_{1}, p_{2}, l_{3}, p_{1}\right) .
$$

Similar to the discussion in (4.27), here we can also split the next-to-minimal form factor $\hat{\mathcal{F}}_{L+1}^{(0)}$ according to their kinematic dependence as

$$
\hat{\mathcal{F}}_{L+1}^{(0)}\left(-l_{1},-l_{2}, A,-l_{3}, B\right)=\hat{\mathcal{F}}_{L+1, \text { dens }}^{(0)}\left(p_{3},-l_{1},-l_{2}, p_{4}\right)+\hat{\mathcal{F}}_{L+1, \mathrm{dens}}^{(0)}\left(p_{5},-l_{3}, p_{6}\right),
$$

and we focus on the first part: ${ }^{10}$

$$
\begin{aligned}
{\left.\left[\mathcal{F}^{(2)}\right]_{\mathcal{T}_{t_{3}}}\right|_{s_{12} \text {-TC }}=} & \int \mathrm{dPS}_{3, l} \hat{\mathcal{F}}_{L+1, \text { dens }}^{(0)}\left(p_{3},-l_{1},-l_{2}, p_{4}\right) \hat{\mathcal{A}}_{5}^{(0)}\left(l_{2}, l_{1}, p_{2}, l_{3}, p_{1}\right) \\
& +\left(\text { kinematic dependence on } p_{5} \text { and } p_{6}\right) .
\end{aligned}
$$

The r.h.s. of (5.18) contains kinematic dependence on $\left\{p_{1}, p_{2}, p_{3}, p_{4}\right\}$, and except $\left[\mathcal{I}^{(2)}(12)\right]_{\check{\mathcal{D}}_{27}}$, all of them have been derived from previous discussion. After subtracting them, we have

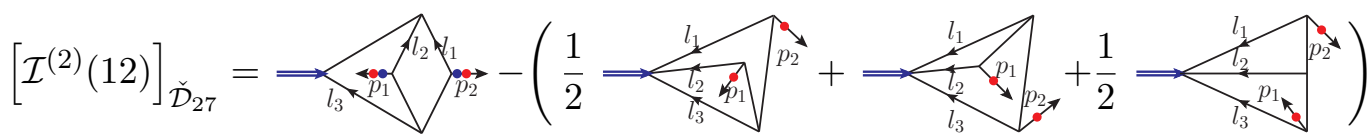

$$
\begin{aligned}
& + \text { (terms detected in other channels). }
\end{aligned}
$$

\footnotetext{
${ }^{10}$ Within this part, the super-density $\hat{\mathcal{F}}_{L+1 \text {,dens }}^{(0)}\left(p_{3},-l_{1},-l_{2}, p_{4}\right)$ allows exchanging gluons in $l_{1}$ or $l_{2}$ line, or a pair of fermions in both lines.
} 


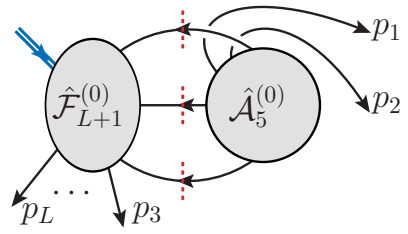

(a) a $s_{12}$ triple-cut.

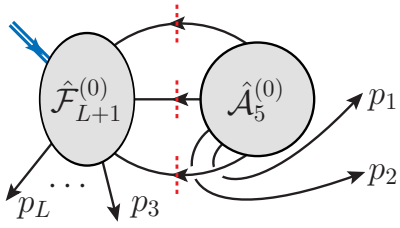

(b) another $s_{12}$ triple-cut.

Figure 9. Selected cuts for $\left[\mathcal{F}^{(2)}\right]_{\mathcal{T}_{t_{4}}}$ for check.

Integrals in (5.27) are put in the form such that the cut structure (figure 8(b)) is manifest. There is another $s_{12}$ triple-cut (figure $8(\mathrm{c})$ ) which can be considered similarly. Details of the calculation can be found in appendix B, where some further features for non-planar unitarity are discussed. After considering this channel, $\left[\mathcal{I}^{(2)}(12)\right]_{\check{\mathcal{D}}_{27}}$ is now complete. The final integral form of $\left[\mathcal{I}^{(2)}(12)\right]_{\check{\mathcal{D}}_{27}}$ is

$$
\left[\mathcal{I}^{(2)}(12)\right]_{\check{\mathcal{D}}_{27}}=-4 \Longrightarrow>_{\operatorname{ap}_{p_{2}}}^{p_{p_{2}}^{p_{1}}}+
$$

Check of other channels. As mentioned above, there are other unitarity channels that we have not used and they can also be checked. Here we present an example. The target color factor is

$$
\mathcal{T}_{t_{4}}=N_{c} \operatorname{tr}\left(a_{1} a_{2}\right) \operatorname{tr}\left(a_{3} \cdots a_{L}\right) .
$$

By extracting $\mathcal{T}_{t_{4}}$ component in (5.1), one finds:

$$
\left.\left(\boldsymbol{I}_{12}^{(2)}+\cdots\right) \boldsymbol{F}^{(0)}\right|_{\mathcal{T}_{t_{4}}}=\left(3\left[\mathcal{I}^{(2)}(12)\right]_{\check{\mathcal{D}}_{25}}+3\left[\mathcal{I}^{(2)}(12)\right]_{\check{\mathcal{D}}_{26}}+2\left[\mathcal{I}^{(2)}(12)\right]_{\check{\mathcal{D}}_{27}}+\cdots\right) \mathcal{F}^{(0)}
$$

where the factor 3 and 2 comes from color structure,

$$
\left.\check{\mathcal{D}}_{25} \operatorname{tr}\left(l_{1} l_{2} a_{3} \cdots a_{L}\right)\right|_{\mathcal{T}_{t_{4}}}=3,\left.\quad \check{\mathcal{D}}_{26} \operatorname{tr}\left(l_{1} l_{2} a_{3} \cdots a_{L}\right)\right|_{\mathcal{T}_{t_{4}}}=3,\left.\quad \check{\mathcal{D}}_{27} \operatorname{tr}\left(l_{1} l_{2} a_{3} \cdots a_{L}\right)\right|_{\mathcal{T}_{t_{4}}}=2 .
$$

We focus on the most constraining cut channels: the $s_{12}$ triple-cut as shown in figure $9:^{11}$

Cut in figure 9(a): $\quad \mathcal{T}_{c}=\operatorname{tr}_{\mathcal{A}}\left(\check{a}_{l_{1}} \check{a}_{l_{3}} \check{a}_{l_{2}} a_{1} a_{2}\right) \times \operatorname{tr}\left(a_{l_{1}} a_{l_{2}} a_{l_{3}} a_{3} \cdots a_{L}\right)$,

$$
\left.\left[\mathcal{F}_{L, 12}^{(2)}\right]_{\mathcal{T}_{c}}\right|_{s_{12}-\mathrm{TC}(\mathrm{a})}=\int \mathrm{dPS}_{3, l} \hat{\mathcal{F}}_{L+1}^{(0)}\left(-l_{1},-l_{2},-l_{3}, p_{3}, \ldots, p_{L}\right) \hat{\mathcal{A}}_{5}^{(0)}\left(l_{1}, l_{3}, l_{2}, p_{1}, p_{2}\right),
$$

Cut in figure 9(b): $\quad \mathcal{T}_{c}=\operatorname{tr}_{\mathcal{A}}\left(\check{a}_{l_{1}} \check{a}_{l_{3}} a_{1} a_{2} \check{a}_{l_{2}}\right) \times \operatorname{tr}_{\mathcal{F}}\left(a_{l_{1}} a_{l_{2}} a_{l_{3}} a_{3} \cdots a_{L}\right)$,

$$
\left.\left[\mathcal{F}_{L, 12}^{(2)}\right]_{\mathcal{T}_{c}}\right|_{s_{12}-\mathrm{TC}(\mathrm{b})}=\int \mathrm{dPS}_{3, l} \hat{\mathcal{F}}_{L+1}^{(0)}\left(-l_{1},-l_{2},-l_{3}, p_{3}, \ldots, p_{L}\right) \hat{\mathcal{A}}_{5}^{(0)}\left(l_{1}, l_{3}, p_{1}, p_{2}, l_{2}\right) .
$$

\footnotetext{
${ }^{11}$ There are two more cut channels not shown in figure 9 which can be obtained by exchanging $p_{1}$ and $p_{2}$ in channel-(a) and (b). There is no need to compute them because both color factor $\mathcal{T}_{t_{4}}$ and kinematic coefficients, the range- 2 integrals, are symmetric between $p_{1}$ and $p_{2}$ so that we just count in an extra 2 factor.
} 
The complete expression is a little lengthy and we only mention that after subtracting cuts from range- 3 contributions, one obtains range-2 integrals as:

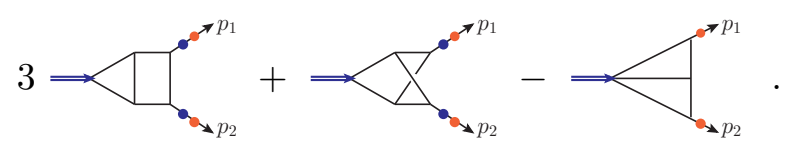

From the unitarity point of view, the factor 3 in the first term can be understood as follows: triple-cut-(a) contains three type of twists of the "planar-ladder" integral

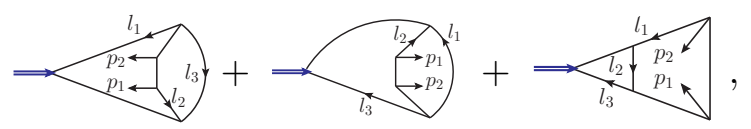

which explains the factor 3 in (5.32). See also the discussion of a similar example in appendix B.

Together with the factors 2 in l.h.s. is explained in Footnote 11, one has

$$
2 \times(5.32)=3\left[\mathcal{I}^{(2)}(12)\right]_{\check{\mathcal{D}}_{25}}+3\left[\mathcal{I}^{(2)}(12)\right]_{\check{\mathcal{D}}_{26}}+2\left[\mathcal{I}^{(2)}(12)\right]_{\check{\mathcal{D}}_{27}},
$$

where (5.19) and (5.28) are used. This is indeed consistent with the wanted result in (5.30).

Summarizing the BPS result. Results for complete correction density functions are summarized in the following equations:

- Range-2:

$$
\begin{aligned}
{\left[\mathcal{I}^{(2)}(12)\right]_{\check{\mathcal{D}}_{25}} } & =\mathcal{I}_{\left.\check{I}^{(2)}(12)\right]_{\check{\mathcal{D}}_{27}}}= \\
\boldsymbol{I}^{(2)} & =\sum_{\sigma \in S_{2}}\left(\check{\mathcal{D}}_{\Delta_{3}(\sigma)}\left[\mathcal{I}^{(2)}\left(\sigma\left(p_{1}, p_{2}\right)\right)\right]_{\check{D}_{25}}\right)+\check{\mathcal{D}}_{\Delta_{4}(\mathbf{1})}^{p_{p_{1}}}\left[\mathcal{I}^{(2)}\left(p_{1}, p_{2}\right)\right]_{\check{D}_{27}}
\end{aligned} .
$$

- Range-3:

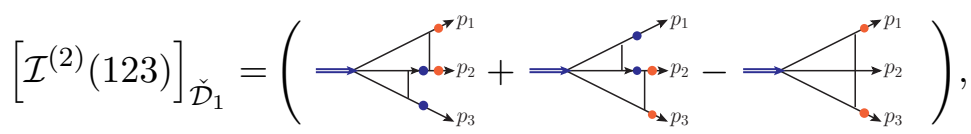

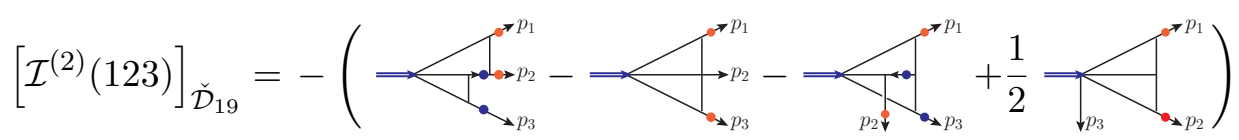

$$
\begin{aligned}
& +\operatorname{cyc}\left(p_{1}, p_{2}, p_{3}\right) \text {, } \\
& \boldsymbol{I}_{123}^{(2)}=\sum_{\sigma^{e} \in S_{3}}\left(\sum_{\sigma^{i} \in \mathbb{Z}_{3}} \check{\mathcal{D}}_{\Delta_{1}\left(\sigma^{e}, \sigma^{i}\right)}\left[\mathcal{I}^{(2)}\left(\sigma^{e}\left(p_{1}, p_{2}, p_{3}\right)\right)\right]_{\check{D}_{1}}\right. \\
& \left.+\check{\mathcal{D}}_{\Delta_{2}\left(\sigma^{e}\right)}\left[\mathcal{I}^{(2)}\left(\sigma^{e}\left(p_{1}, p_{2}, p_{3}\right)\right)\right]_{\check{D}_{19}}\right) .
\end{aligned}
$$


Below we show that the above result can be given in a much simpler form. In particular, the range-3 non-planar integrals can all be reduced to planar ones.

Cancellation of range-3 non-planar integrals. Results in (5.36a)-(5.36b) can actually be simplified further with an integral relation. This is inspired by [86] where it was found that all non-planar range-3 Wilson line integrals do not exist in the final result. Although the objects considered there are Wilson lines, one may expect the similar pattern to appear also in form factors. Indeed, we find that the range-3 non-planar integrals, although having non-trivial integrand, can be reduced to a linear combination of planar integrals.

The three non-planar integrals in (5.36b) can be written together as

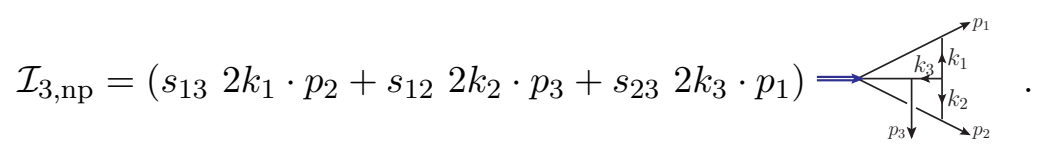

As proved in appendix $\mathrm{E}, \mathcal{I}_{3, \mathrm{np}}$ has an implicit parity symmetry, and it is then easy to show that

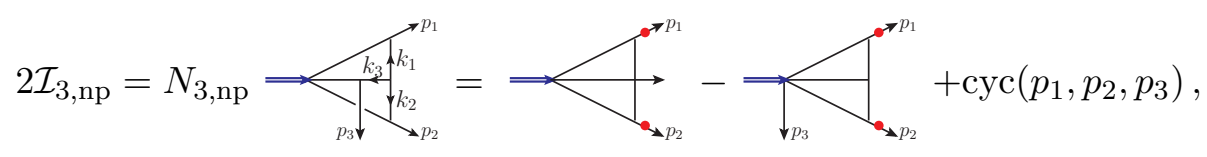

in which only planar integrals are left, and

$$
N_{3, \mathrm{np}}=\left(s_{12}\left(k_{3}+p_{3}\right)^{2}-s_{12} k_{3}^{2}+s_{13}\left(k_{2}+p_{2}\right)^{2}-s_{13} k_{2}^{2}+s_{23}\left(k_{1}+p_{1}\right)^{2}-s_{23} k_{1}^{2}\right) .
$$

Using (5.38), one can rewrite (5.36b) in a much simpler form as

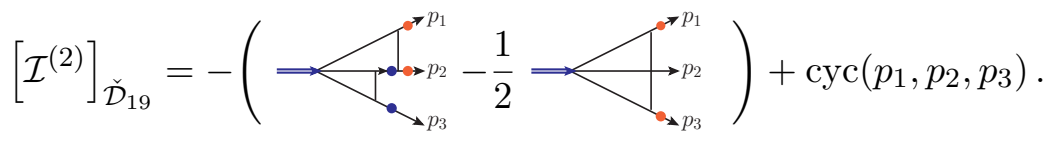

As a comparison, let us recall (5.36a)

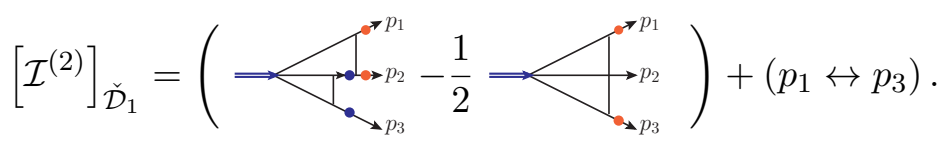

We can see that range-3 BPS densities can be expressed via only one building block after using integral relations aforementioned. ${ }^{12}$

\footnotetext{
${ }^{12}$ The similarity of the above two forms, together with color identity given in (A.2), allow us to express the full-color range-3 density as

$$
\boldsymbol{I}_{123}^{(2)}=\sum_{\sigma^{e}, \sigma^{i} \in S_{3}} \check{\mathcal{D}}_{\Delta_{1}}\left(\sigma^{e}, \sigma^{i}\right) \times \mathcal{I}_{\mathrm{BPS}, \mathrm{a}}^{(2)}\left(\sigma^{e}\left(p_{1}, p_{2}, p_{3}\right)\right),
$$

where $\mathcal{I}_{\mathrm{BPS}, \mathrm{a}}^{(2)}$ are the terms in the parenthesis of (5.40)-(5.41) (see also (7.23)).
} 


\subsection{Two-loop SU(2) correction}

In this subsection, we employ the method aforementioned to non-BPS form factors with operators in the $\mathrm{SU}(2)$ sector. The color decomposition in section 5.1 applies here, and one can still focus on the $\left\{\left[\mathcal{I}_{123}^{(2)}\right]_{\check{\mathcal{D}}_{1}},\left[\mathcal{I}_{123}^{(2)}\right]_{\check{\mathcal{D}}_{19}},\left[\mathcal{I}_{123}^{(2)}\right]_{\check{\mathcal{D}}_{25}},\left[\mathcal{I}_{123}^{(2)}\right]_{\check{\mathcal{D}}_{27}}\right\}$

Unlike the BPS case, in $\mathrm{SU}(2)$ sector there are two types of fields $X$ and $Y$, so the corresponding density functions have more than one field configurations. One should therefore take into account all inequivalent configurations. In this calculation, the Super-WardIdentity with respect to $\mathrm{SU}(2)$ R-symmetry can provide a useful simplification, which have been observed in [38] in the planar case and will be generalized to full-color case in our calculation. Using these relations, we can identify a small set of kinematic functions with specific field configurations that one needs to compute.

SU(2) supersymmetric relations. We first review the planar case. All possible field configurations can be given as follows: ${ }^{13}$

- range-2: $Y X \rightarrow Y X, Y X \rightarrow X Y$ and reverting the order of the fields. The total number of configurations is 4 .

- range-3: $Y X X \rightarrow Y X X, X Y X \rightarrow X Y X, Y X X \rightarrow X Y X, X Y X \rightarrow Y X X$ and $Y X X \rightarrow X X Y$ and reverting the order of the fields. The configuration number is 9.

Supersymmetry provides symmetric constraint on form factors via Super-WardIdentity:

$$
0=\langle\Phi(1) \cdots \Phi(n)[\mathfrak{S}, \mathcal{O}]\rangle+\sum_{i=1}^{n}\langle\Phi(1) \cdots[\mathfrak{S}, \Phi(i)] \cdots \Phi(n) \mathcal{O}\rangle,
$$

where $\Phi$ can be general on-shell superfield or component fields and $\mathfrak{S}$ is a supersymmety generator, which can be taken as R-charge generators $R_{B}^{A}$ in our particular discussion on $\mathrm{SU}(2)$ form factors. As a result, planar $\mathrm{SU}(2)$ relations have the following form in general

$$
\begin{aligned}
\sum_{\tau \in \mathbb{Z}_{2}}\left[\left(\mathcal{I}^{(2)}\right)_{Y X}^{\tau(Y X)}\right]_{\mathrm{PL}} & =\left[\left(\mathcal{I}^{(2)}\right)_{X X}^{X X}\right]_{\mathrm{PL}}, \\
\sum_{\tau_{a} \in \mathbb{Z}_{3}}\left[\left(\mathcal{I}^{(2)}\right)_{\tau_{a}(Y X X)}^{\tau_{b}(Y X X)}\right]_{\mathrm{PL}} & =\left[\left(\mathcal{I}^{(2)}\right)_{X X X}^{X X X}\right]_{\mathrm{PL}}, \quad \forall \tau_{b} \in \mathbb{Z}_{3}, \\
\sum_{\tau_{b} \in \mathbb{Z}_{3}}\left[\left(\mathcal{I}^{(2)}\right)_{\tau_{a}(Y X X)}^{\tau_{b}(Y X X)}\right]_{\mathrm{PL}} & =\left[\left(\mathcal{I}^{(2)}\right)_{X X X}^{X X X}\right]_{\mathrm{PL}}, \quad \forall \tau_{a} \in \mathbb{Z}_{3} .
\end{aligned}
$$

Note that unlike in (5.9)-(5.10), here the permutation $\tau$ acts only on fields (not on momenta). Also, planar densities satisfy the reflection property:

$$
\left[\left(\mathcal{I}^{(2)}\right)_{A^{\prime} B^{\prime} C^{\prime}}^{A B C}\right]_{\mathrm{PL}}\left(p_{1}, p_{2}, p_{3}\right)=\left[\left(\mathcal{I}^{(2)}\right)_{C^{\prime} B^{\prime} A^{\prime}}^{C B A}\right]_{\mathrm{PL}}\left(p_{3}, p_{2}, p_{1}\right)
$$

In the end, it turns out that one only needs to compute

\footnotetext{
${ }^{13}$ The density involving two $Y$ fields can be simply obtained by exchanging $X$ and $Y$ fields, and we will not consider them.
} 
- range-2: $Y X \rightarrow Y X$,

- range-3: $Y X X \rightarrow Y X X, Y X X \rightarrow X X Y$,

to get all planar results, see also [38] for more details.

Next we consider to the full-color case. It is straightforward to generalize (5.43) as

$$
\begin{aligned}
\sum_{\tau \in \mathbb{Z}_{2}}\left(\boldsymbol{I}_{12}^{(2)}\right)_{Y X}^{\tau(Y X)} & =\left(\boldsymbol{I}_{12}^{(2)}\right)_{X X}^{X X}, \\
\sum_{\tau_{a} \in \mathbb{Z}_{3}}\left(\boldsymbol{I}_{123}^{(2)}\right)_{\tau_{a}(Y X X)}^{\tau_{b}(Y X X)} & =\left(\boldsymbol{I}_{123}^{(2)}\right)_{X X X}^{X X X}, \quad \forall \tau_{b} \in \mathbb{Z}_{3}, \\
\sum_{\tau_{b} \in \mathbb{Z}_{3}}\left(\boldsymbol{I}_{123}^{(2)}\right)_{\tau_{a}(Y X X)}^{\tau_{b}(Y X X)} & =\left(\boldsymbol{I}_{123}^{(2)}\right)_{X X X}^{X X X}, \quad \forall \tau_{a} \in \mathbb{Z}_{3} .
\end{aligned}
$$

By expanding (5.45) to a set of color basis, for example the trivalent basis in section 5.1, one also has the $\mathrm{SU}(2)$ relations for color-stripped densities|just replace $\boldsymbol{I}$ with $[\mathcal{I}]_{\check{\mathcal{D}}_{i}}$ for some $\check{\mathcal{D}}_{i}$.

As mentioned, we only need to focus on the densities with $\check{\mathcal{D}}_{i}, i=1,19,25,27$, which we describe respectively below:

1. For $\left[\mathcal{I}^{(2)}\right]_{\check{\mathcal{D}}_{1}}$, our experience in BPS computation shows that they can be mapped to the planar case, and the independent matrix elements can be also chosen as the planar ones.

2. For $\left[\mathcal{I}^{(2)}\right]_{\check{\mathcal{D}}_{19}}$, because of the cyclic symmetry of color factor $\check{D}_{19}$, we have

$$
\left[\left(\mathcal{I}^{(2)}\right)_{\sigma(Y X X)}^{\sigma \tau(Y X X)}\left(\sigma\left(p_{1}, p_{2}, p_{3}\right)\right)\right]_{\check{\mathcal{D}}_{19}}=\left[\left(\mathcal{I}^{(2)}\right)_{Y X X}^{\tau(Y X X)}\left(p_{1}, p_{2}, p_{3}\right)\right]_{\check{\mathcal{D}}_{19}}, \quad \forall \tau, \sigma \in \mathbb{Z}_{3} .
$$

Thus, the nine density functions for $\left[\mathcal{I}^{(2)}\right]_{\check{\mathcal{D}}_{19}}$ are reduced to only three ones in r.h.s. of (5.46). And the $\mathrm{SU}(2)$ relation from (5.45) involving only these three functions is

$$
\sum_{\tau \in \mathbb{Z}_{3}}\left[\left(\mathcal{I}^{(2)}\right)_{Y X X}^{\tau(Y X X)}\right]_{\check{\mathcal{D}}_{19}}=\left[\left(\mathcal{I}^{(2)}\right)_{X X X}^{X X X}\right]_{\check{\mathcal{D}}_{19}} .
$$

So we can further select two of these three functions as independent basis.

3. For $\left[\mathcal{I}^{(2)}\right]_{\check{\mathcal{D}}_{25}}$ or $\left[\mathcal{I}^{(2)}\right]_{\check{\mathcal{D}}_{27}}$, invariance of density function under flipping $X$ and $Y$ fields reduces four configurations to two. And the following $\mathrm{SU}(2)$ equations

$$
\left[\left(\mathcal{I}^{(2)}\right)_{Y X}^{Y X}\right]_{\check{\mathcal{D}}_{j}}+\left[\left(\mathcal{I}^{(2)}\right)_{Y X}^{X Y}\right]_{\check{\mathcal{D}}_{j}}=\left[\left(\mathcal{I}^{(2)}\right)_{X X}^{X X}\right]_{\check{\mathcal{D}}_{j}}, \quad j=25,27,
$$

lead to the conclusion that $Y X \rightarrow Y X$ is sufficient.

To summarize, the independent matrix elements for $\mathrm{SU}(2)$ form factors can be chosen as

$$
\begin{aligned}
& {\left[\mathcal{I}^{(2)}\right]_{\check{\mathcal{D}}_{1}}: \quad Y X X \rightarrow Y X X, Y X X \rightarrow X X Y, } \\
& {\left[\mathcal{I}^{(2)}\right]_{\check{\mathcal{D}}_{19}}: \quad Y X X \rightarrow Y X X, Y X X \rightarrow X Y X, } \\
{\left[\mathcal{I}^{(2)}\right]_{\check{\mathcal{D}}_{25}} \text { and }\left[\mathcal{I}^{(2)}\right]_{\check{\mathcal{D}}_{27}}: \quad Y X \rightarrow Y X . } &
\end{aligned}
$$


Unitarity construction. Having identified the target density functions, we can use the unitarity method to construct them. The unitarity computation for these building blocks is totally parallel to the BPS case. The only change is that the tree building blocks contain both $X, Y$ as external states. The six-point NMHV scalar amplitudes with $X, Y, \bar{X}$ and $\bar{Y}$ as external states are major building blocks, which are explicitly given in appendix C. We will not reproduce the details of the calculation and just summarize the final results below:

- Range-2:

$$
\begin{aligned}
& {\left[\left(\mathcal{I}^{(2)}\right)_{Y X}^{Y X}\right]_{\mathcal{D}_{25}}=(\Longrightarrow \overbrace{p_{p_{p}}}^{p_{p_{2}}}+2} \\
& {\left[\left(\mathcal{I}^{(2)}\right)_{Y X}^{Y X}\right]_{\mathcal{D}_{27}}=-\frac{1}{2}=(5.35 \mathrm{a}),}
\end{aligned}
$$

where the second equation in $(5.50 \mathrm{~b})$ results from

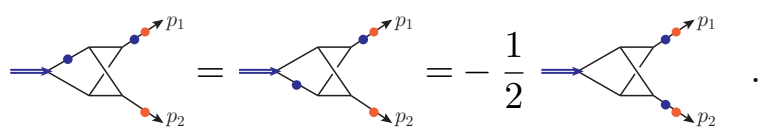

- Range-3:

$$
\begin{aligned}
& {\left[\left(\mathcal{I}^{(2)}\right)_{Y X X}^{Y X X}\right]_{\mathcal{D}_{1}}=(\overbrace{p_{p_{3}}}^{p_{p_{1}}}+\overbrace{p_{2} p_{p_{3}}}^{p_{1}})+(5.36 \mathrm{a}),} \\
& {\left[\left(\mathcal{I}^{(2)}\right)_{Y X X}^{X X Y}\right]_{\mathcal{D}_{1}}=(\overbrace{p_{p_{3}}^{p_{2}}}^{p_{1}})}
\end{aligned}
$$

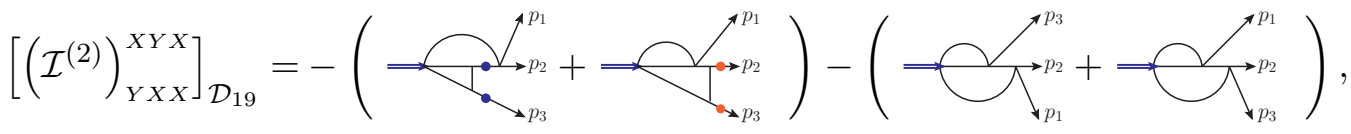

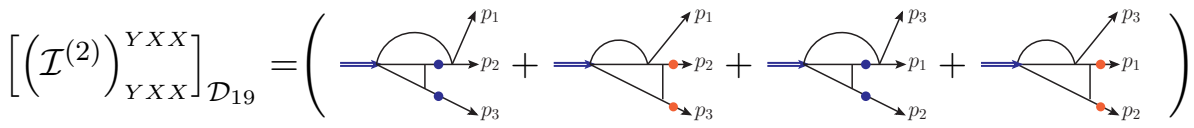

$$
\begin{aligned}
& +\left(ح_{p_{1}}^{p_{3}} p_{p_{2}}\right)+(5.36 \mathrm{~b}) \text {. }
\end{aligned}
$$

From (5.50a)-(5.52d), one can see that it is possible to separate the BPS part as

$$
\left[\left(\tilde{\mathcal{I}}^{(2)}\right)_{\mathbf{w}_{i}}^{\mathbf{w}_{i}^{\prime}}\right]_{\check{\mathcal{D}}_{i}}=\left[\left(\mathcal{I}^{(2)}\right)_{\mathbf{w}_{i}}^{\mathbf{w}_{i}^{\prime}}\right]_{\check{\mathcal{D}}_{i}}-\delta_{\mathbf{w}_{i}}^{\mathbf{W}_{i}^{\prime}}\left[\left(\mathcal{I}_{\mathrm{BPS}}^{(2)}\right)_{X \cdots X}^{X \cdots X}\right]_{\check{\mathcal{D}}_{i}},
$$

with $i=1,19,25,27$ as our focused elements. As will be discussed in section 7.3, it is possible to further separate the IR and UV divergences in a manifest way. 


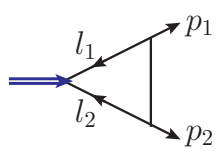

(a) triangle

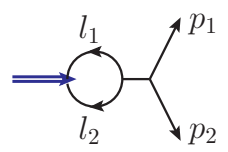

(b) bubble

Figure 10. Trivalent topology for one-loop minimal BPS form factors.

\section{Color-kinematics duality for two-loop BPS form factors}

In this section, we study non-planar form factors with the method of the color-kinematics duality. As a concrete result, we will reconstruct the two-loop BPS form factor and obtain a representation satisfying CK duality at the loop density level.

Before discussing two loops, it should be helpful to have a simple check at one-loop level. There are two types of trivalent topologies at one-loop level as shown in figure 10 . For BPS form factors, the existence of one-loop CK-dual representation is easy to see, where the planar kinematic part is (see appendix D for discussions and notations)

$$
\mathcal{I}_{\mathrm{BPS}}^{(1)}(12)=-\mathrm{I}_{\mathrm{tri}}\left[s_{12}\right],
$$

and the full-color one-loop correction is

$$
\boldsymbol{I}_{12, \mathrm{BPS}}^{(1)}=-\tilde{f}^{\check{a}_{l_{1}} a_{1} \mathrm{x}} \tilde{f} \tilde{\mathrm{x}}^{\mathrm{x} a_{2} \check{a}_{l_{2}}} s_{12} \Longrightarrow \overbrace{l_{2}}^{l_{1}} \int_{p_{2}}^{p_{1}}-\tilde{f}^{\tilde{a}_{l_{1}} a_{2} \mathrm{x}} \tilde{f} \tilde{f}^{\mathrm{x} a_{1} \check{a}_{l_{2}}} s_{12}
$$

Obviously, such a quantity satisfies CK duality with $N_{\text {tri }}=-s_{12}$ and $N_{\text {bub }}=0$. Similar construction can be also generalized to non-BPS operators, and the explicit CK-dual form for one-loop $\mathrm{SU}(2)$ form factors is provided in appendix D. We leave more general discussions to another paper.

As we will show in this section, the two-loop generalization of CK duality is also possible. Following section 3.3, below we first use a six-point tree amplitude as an example to illustrate the construction. Then we go on to construct the two-loop BPS form factors.

\subsection{Six-scalar tree amplitude}

As a warm up, we describe the calculation of CK-dual representation for the full-color sixpoint amplitude $\boldsymbol{A}_{6}^{(0)}\left(p_{1}^{X}, p_{2}^{X}, p_{3}^{X}, q_{1}^{\bar{X}}, q_{2}^{\bar{X}}, q_{3}^{\bar{X}}\right)$. Via unitarity cut, this six-point amplitude is also a building block in the two-loop BPS form factor. Following (3.31), we expect the six scalar amplitude to be given in the following form:

$$
\boldsymbol{A}_{6}^{(0)}\left(p_{1}^{X}, p_{2}^{X}, p_{3}^{X}, q_{1}^{\bar{X}}, q_{2}^{\bar{X}}, q_{3}^{\bar{X}}\right)=\sum_{\sigma} \sum_{\Gamma_{i}} \frac{1}{S_{i}} \frac{\mathcal{C}_{i} N_{i}}{\prod_{a} d_{i, a}}
$$

We can construct this form in the following steps: 


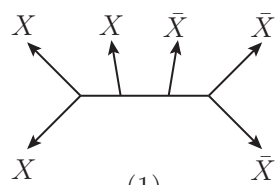

(1)

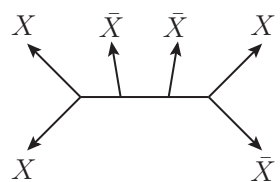

(5)

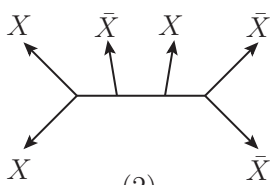

(2)

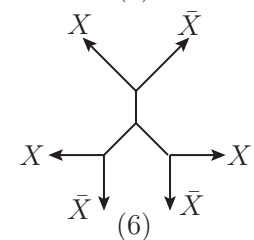

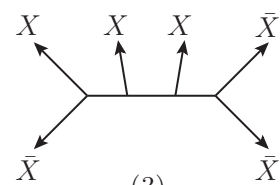

(3)

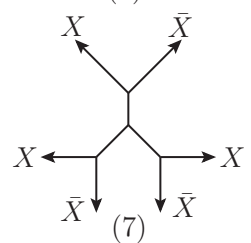

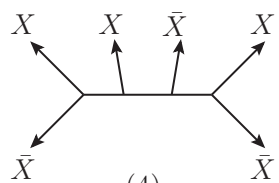

(4)

Figure 11. Topology with scalar amplitudes with $X$ and $\bar{X}$ external states, labeled as $\Gamma_{i}$. Only $\Gamma_{4}$ and $\Gamma_{6}$ give non-vanishing numerators.

\section{Generating trivalent diagrams $\Gamma_{i}$.}

The trivalent topologies of inequivalent external scalar configurations are given in figure 11. Note that we do not need to specify the momenta in this step; hence permutations of $\left\{p_{1}, p_{2}, p_{3}\right\}$ will not lead to different trivalent diagrams $\Gamma_{i}$.

2. Dual Jacobi relation and master topology.

Dual Jacobi relations like (3.37) provide connections between numerators, for example:

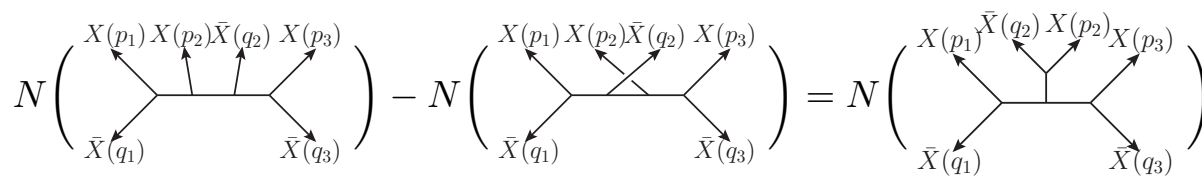

$$
N_{4}\left(p_{1}, p_{2}, p_{3}, q_{1}, q_{2}, q_{3}\right)-N_{4}\left(p_{3}, p_{2}, p_{1}, q_{3}, q_{2}, q_{1}\right)=N_{6}\left(p_{1}, p_{2}, p_{3}, q_{1}, q_{2}, q_{3}\right),
$$

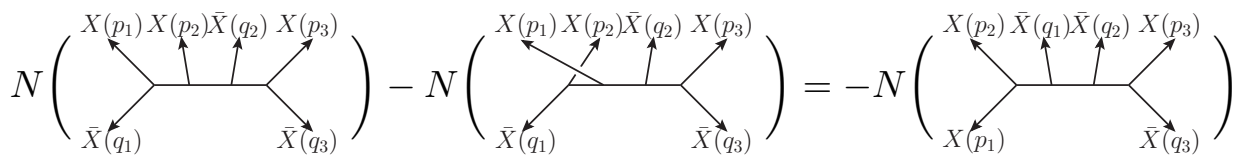

$$
N_{4}\left(p_{1}, p_{2}, p_{3}, q_{1}, q_{2}, q_{3}\right)-N_{4}\left(p_{2}, p_{1}, p_{3}, q_{1}, q_{2}, q_{3}\right)=-N_{5}\left(p_{1}, p_{2}, p_{3}, q_{1}, q_{2}, q_{3}\right) .
$$

Using these identities, one finds out that figure 11(4) can be chosen as the master topology and other numerators can be expressed by linear combination of $N_{4}$.

3. Ansatz and constraint for master numerator

Before writing down an ansatz for the master numerator $N_{4}$, let us consider the following two constrains:

(a) We assume that one can set all numerators of diagrams with two external $X$ or $\bar{X}$ connected to the same vertex zero. This directly means that except the numerators of (4) and (6) in figure 11, all other numerators are zero. Due to 
the fact that $N_{3}=0, N_{4}$ should be symmetric by shifting the first two or the last two arguments, such as $N_{4}\left(p_{1}, p_{2}, p_{3}, q_{1}, q_{2}, q_{3}\right)=N_{4}\left(p_{1}, p_{2}, p_{3}, q_{1}, q_{3}, q_{2}\right)$.

(b) Then we use a simple cut condition, by setting the center propagator $\left(q_{1}+p_{1}+\right.$ $\left.p_{2}\right)^{2} \rightarrow 0$. This cut channel can actually detects topology (2)-(5), but as we mentioned that only (4) is non-vanishing. Under this cut, six point amplitudes factorize into product of two four point amplitudes, with $P \equiv p_{1}+p_{2}+q_{1}$

$$
\begin{aligned}
& \left.\left(P^{2} \boldsymbol{A}_{6}^{(0)}\left(p_{1}^{X}, p_{2}^{X}, p_{3}^{X}, q_{1}^{\bar{X}}, q_{2}^{\bar{X}}, q_{3}^{\bar{X}}\right)\right)\right|_{P^{2} \rightarrow 0} \\
& \quad=\boldsymbol{A}_{4}^{(0)}\left(q_{1}^{\bar{X}}, p_{1}^{X}, p_{2}^{X}, P^{\bar{X}}\right) \boldsymbol{A}_{4}^{(0)}\left(q_{2}^{\bar{X}}, q_{3}^{\bar{X}}, p_{3}^{X},-P^{X}\right),
\end{aligned}
$$

with this condition, we see that

$$
\left.N_{4}\left(p_{1}, p_{2}, p_{3}, q_{1}, q_{2}, q_{3}\right)\right|_{P^{2} \rightarrow 0}=-s_{p_{1} p_{2}} s_{q_{2} q_{3}},
$$

where the minus sign on r.h.s. comes from the color factor.

Given the above two constraints, an ansatz for $N_{4}$ can be given as ${ }^{14}$

$$
N_{4}\left(p_{1}, p_{2}, p_{3}, q_{1}, q_{2}, q_{3}\right)=-s_{p_{1} p_{2}} s_{q_{2} q_{3}}+s_{q_{1} p_{1} p_{2}} \sum_{i=1}^{4} \beta_{i} u_{i},
$$

where $\beta_{i}$ are numerical parameters to be further determined and

$$
u_{i} \in\left\{s_{p_{1} p_{2}}+s_{q_{2} q_{3}}, s_{p_{3} q_{1}}, s_{p_{1} p_{2} p_{3}}, s_{p_{1} p_{2} q_{1}}\right\}
$$

are independent lorentz products symmetric under both $\left(p_{1} \leftrightarrow p_{2}\right)$ and $\left(q_{2} \leftrightarrow q_{3}\right)$.

\section{Symmetry of numerators.}

From the master ansatz (6.8), all other numerators can be obtained through dual Jacobi relations. We then impose the condition that the numerators should have symmetries inherited from symmetries of corresponding topology.

For the two non-vanishing diagram, one can see that diagram figure 11(4) has no intrinsic symmetry, while diagram figure 11(6) has cyclic and parity symmetry and its symmetric factor is 6 . As a result, one has

$$
N_{6}\left(p_{1}, p_{2}, p_{3}, q_{1}, q_{2}, q_{3}\right)= \pm N_{6}\left(\sigma\left\{p_{1}, p_{2}, p_{3}\right\}, \sigma\left\{q_{1}, q_{2}, q_{3}\right\}\right), \quad \text { with } \sigma \in S_{3} .
$$

To determine the sign, we use the following color convention:

$$
\mathcal{C}_{6}\left(p_{1}, p_{2}, p_{3}, q_{1}, q_{2}, q_{3}\right)=(-1)^{\sigma} \mathcal{C}_{6}\left(\sigma\left\{p_{1}, p_{2}, p_{3}\right\}, \sigma\left\{q_{1}, q_{2}, q_{3}\right\}\right), \quad \sigma \in S_{3} .
$$

Thus, we have the following relation for $N_{6}$

$$
N_{6}\left(p_{1}, p_{2}, p_{3}, q_{1}, q_{2}, q_{3}\right)=(-1)^{\sigma} N_{6}\left(\sigma\left\{p_{1}, p_{2}, p_{3}\right\}, \sigma\left\{q_{1}, q_{2}, q_{3}\right\}\right), \quad \sigma \in S_{3} .
$$

\footnotetext{
${ }^{14}$ In this ansatz we also make the local numerator assumption, i.e. the numerators are only polynomials of Mandelstam variables.
} 


\begin{tabular}{|l|r|r|}
\hline$\Gamma_{i}$ & $N_{i}$ & $S_{i}$ \\
\hline & $-s_{p_{1} p_{2}} s_{q_{2} q_{3}}+\frac{1}{2} s_{p_{1} p_{2} p_{3}} s_{p_{1} p_{2} q_{1}}$ & 1 \\
\hline
\end{tabular}

Table 3. Various coefficients for the CK-dual tree scalar amplitudes. The "cyc." in the second numerator represents cyclically permuting $\left\{p_{1}, p_{2}, p_{3}\right\}$ and $\left\{q_{1}, q_{2}, q_{3}\right\}$ simultaneously. The color factors $\mathcal{C}_{i}$ can be directly read out from diagrams, given the rule that each vertex gives a $f^{a b c}$ with legs $a, b, c$ arranged in clockwise order.

This symmetry condition turns out to be enough to fix all the parameters $\beta_{i}$, and the only non-vanishing parameter is

$$
\beta_{3}=\frac{1}{2} .
$$

Thus, the master numerator (6.8) is

$$
-s_{p_{1} p_{2}} s_{q_{2} q_{3}}+\frac{1}{2} s_{p_{1} p_{2} p_{3}} s_{p_{1} p_{2} q_{1}} .
$$

5. Analytic check.

Finally, we write down the full tree amplitude as

$$
\boldsymbol{A}_{6}^{(0)}=\sum_{\sigma_{1}, \sigma_{2}} \sum_{\Gamma_{i}} \frac{1}{S_{i}} \frac{\mathcal{C}_{i}\left(\sigma_{1}, \sigma_{2}\right) N_{i}\left(\sigma_{1}, \sigma_{2}\right)}{\prod_{a} d_{i, a}\left(\sigma_{1}, \sigma_{2}\right)},
$$

where $\sigma_{1}, \sigma_{2}$ are $S_{3}$ permutations of three $X$ and three $\bar{X}$ respectively. The various factors are summarized in table 3 .

We have checked that (6.14) is indeed equivalent to the result from direct computations. For example, one can expand the full-color amplitudes to color-ordered amplitudes and compare those with the expressions in appendix C.

It is worthwhile to mention that under the conditions that we have imposed, the solution is unique. Through unitarity cut, the six-point amplitude will be an important building block in the two-loop BPS form factor, thus (6.14) will serve as a useful input when we consider two-loop range-3 density.

\subsection{Two-loop CK-dual representation}

Now we construct the CK-dual representation for the two-loop half-BPS form factor of $\operatorname{tr}\left(X^{L}\right)$. As explained in section 3.3, one only needs to focus on the loop density functions. The strategy of construction is similar to the aforementioned one, and we will sketch the steps and highlight the difference. 


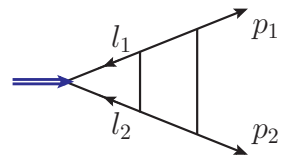

(a)

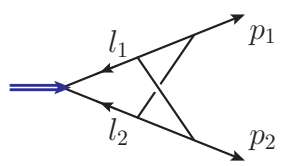

(b)

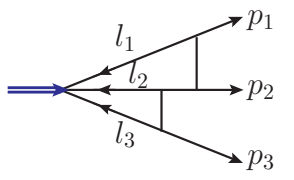

(c)

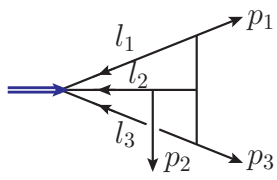

(d)

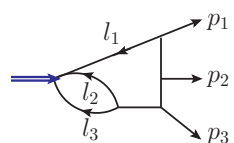

(e)

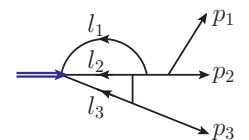

(f)

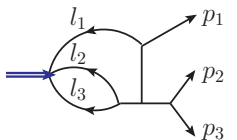

$(\mathrm{g})$

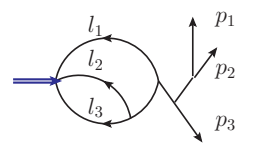

(h)

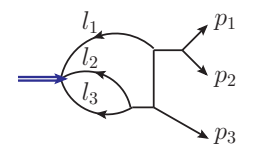

(i)

Figure 12. Trivalent topologies that contribute to BPS form factors. The numerator functions are defined according the labeling of momenta as functions $N_{i}\left(p_{1}, p_{2}, l_{1}, l_{2}\right)$ for $i \in\{\mathrm{a}, \mathrm{b}\}$, and $N_{i}\left(p_{1}, p_{2}, p_{3}, l_{1}, l_{2}, l_{3}\right)$ for $i \in\{\mathrm{c}, \mathrm{d}, \mathrm{e}, \mathrm{f}, \mathrm{g}, \mathrm{h}, \mathrm{i}\}$. Color factors are defined with the rule that each vertex gives a $\tilde{f}^{a b c}$ with legs $a, b, c$ arranged in clockwise order according to the figures. The four topologies in the first row represent, essentially, the four types of non-vanishing integral contributions while the topologies in the second row will disappear after integration.

\section{Generating trivalent topologies.}

It is convenient to separate the two-loop topologies into two classes: the range-2 integrals and range-3 integrals. Due to the difference of color structures, these two classes are decoupled with each other under Jacobi relations, see the discussion in section 3.3. Samples of trivalent topologies are given in figure 12. Within these figures, only graphs with finally non-vanishing numerators are listed.

\section{Dual Jacobi relation and master topology.}

After generating and applying the dual Jacobi relations, the master topologies can be chosen as figure 12(a) and figure 12(c).

A set of dual Jacobi relations are:

$$
\begin{array}{ll}
(1): & N_{\mathrm{c}}\left(p_{1}, p_{2}, p_{3}, l_{1}, l_{2}, l_{3}\right)-N_{\mathrm{c}}\left(p_{3}, p_{2}, p_{1}, l_{3}, l_{2}, l_{1}\right)=N_{\mathrm{d}}\left(p_{1}, p_{2}, p_{3}, l_{1}, l_{2}, l_{3}\right) \\
(2): & N_{\mathrm{c}}\left(p_{1}, p_{2}, p_{3}, l_{1}, l_{2}, l_{3}\right)-N_{\mathrm{c}}\left(p_{1}, p_{2}, p_{3}, l_{1}, l_{3}, l_{2}\right)=N_{\mathrm{e}}\left(p_{1}, p_{2}, p_{3}, l_{1}, l_{2}, l_{3}\right) \\
(3): & N_{\mathrm{c}}\left(p_{1}, p_{2}, p_{3}, l_{1}, l_{2}, l_{3}\right)-N_{\mathrm{c}}\left(p_{2}, p_{1}, p_{3}, l_{1}, l_{2}, l_{3}\right)=N_{\mathrm{f}}\left(p_{1}, p_{2}, p_{3}, l_{1}, l_{2}, l_{3}\right) \\
(4): & N_{\mathrm{e}}\left(p_{1}, p_{2}, p_{3}, l_{1}, l_{2}, l_{3}\right)-N_{\mathrm{e}}\left(p_{1}, p_{3}, p_{2}, l_{1}, l_{3}, l_{2}\right)=N_{\mathrm{g}}\left(p_{1}, p_{2}, p_{3}, l_{1}, l_{2}, l_{3}\right) \\
(5): & N_{\mathrm{e}}\left(p_{1}, p_{2}, p_{3}, l_{1}, l_{2}, l_{3}\right)-N_{\mathrm{e}}\left(p_{2}, p_{1}, p_{3}, l_{1}, l_{3}, l_{2}\right)=N_{\mathrm{i}}\left(p_{1}, p_{2}, p_{3}, l_{1}, l_{2}, l_{3}\right) \\
(6): & N_{\mathrm{g}}\left(p_{3}, p_{1}, p_{2}, l_{1}, l_{2}, l_{3}\right)-N_{\mathrm{i}}\left(p_{1}, p_{2}, p_{3}, l_{1}, l_{3}, l_{2}\right)=N_{\mathrm{h}}\left(p_{1}, p_{2}, p_{3}, l_{1}, l_{2}, l_{3}\right) .
\end{array}
$$

Diagrammatic representation of these relations are given in figure 13.

We would like to mention that, among these equations, the last five equations involve certain special integrals as will be discussed later: diagrams (e)-(i) have non-vanishing numerators but are actually zero after integration and hence do not contribute to the final form factor. 

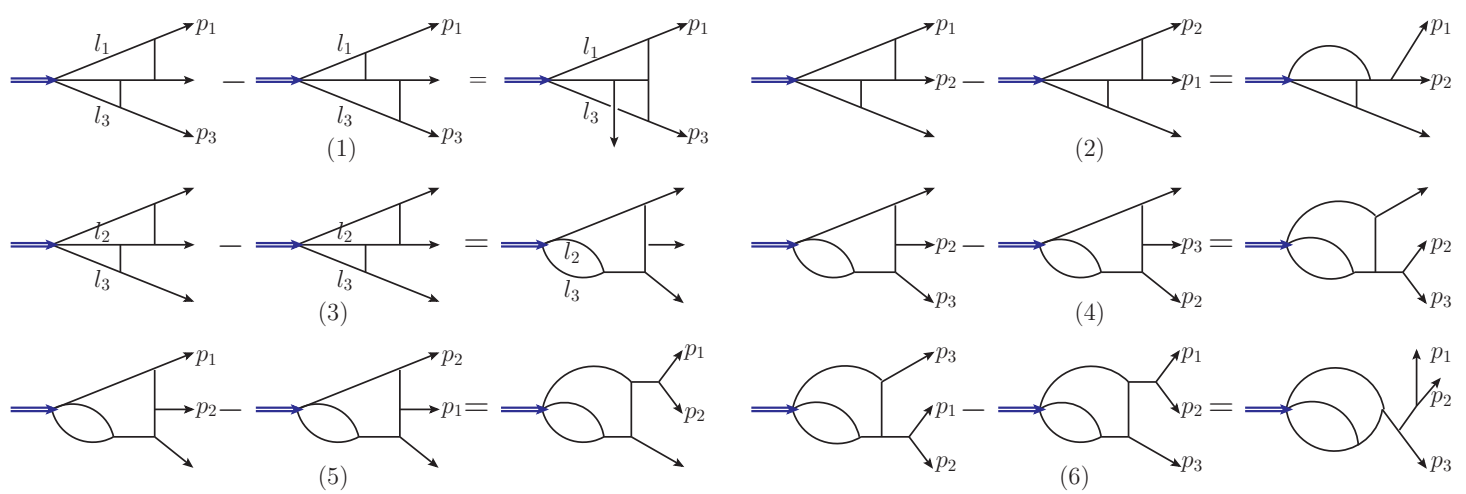

Figure 13. Diagrammatic representation of dual Jacobi relations in (6.15).

\section{Constraint and ansatz for master numerators.}

Since the half-BPS form factor have a good UV behavior, one may expect the following power counting properties for the numerators:

- For any $n$-point one-loop sub-diagram, if this sub-diagram does not contains $q^{2}$ vertex, then the power of loop momenta should not exceed $n-4 .^{15}$

- On the other hand, if this one-loop sub-diagram is a form factor containing the $q^{2}$ vertex, then the naive power counting shows that power of loop momenta should be less than or equal to $n-3$.

This argument can directly exclude sub-bubble or sub-triangle diagrams, except that the triangle is connected to $q^{2}$ vertex. Such a naive constraint will set numerators of (e)-(i) in figure 12 to be zero. However, as will be seen later, this constraint is too strong. To relax the constraint, we enlarge this ansatz to include terms proportional to $l_{k}^{2}$. This allows all of the diagrams explicitly listed in figure 12 to be non-vanishing.

With these considerations, we propose the following ansatz form for two master numerators: for range-2, the master numerator can be nothing but

$$
N_{\mathrm{a}}\left(p_{1}, p_{2}\right)=s_{12}^{2},
$$

and for range-3, we write the numerator as

$$
N_{\mathrm{c}}\left(p_{1}, p_{2}, p_{3}, l_{1}, l_{2}, l_{3}\right)=s_{p_{1} p_{2}} s_{q_{2} q_{3}}-\frac{1}{2} s_{p_{1} p_{2} p_{3}} s_{p_{1} p_{2} q_{1}}+\sum_{i=j}^{9} c_{j} v_{j}
$$

where $c_{j}$ are numerical parameters to be fixed and

$$
v_{i}=\left\{s_{12} l_{1}^{2}, s_{13} l_{1}^{2}, s_{23} l_{1}^{2}, s_{12} l_{2}^{2}, s_{13} l_{2}^{2}, s_{23} l_{2}^{2}, s_{12} l_{3}^{2}, s_{13} l_{3}^{2}, s_{23} l_{3}^{2}\right\} .
$$

Note that the ansatz form in (6.17) results from a triple cut, involving the CK-dual form of tree-level six point amplitudes in table 3, which is the building block of

\footnotetext{
${ }^{15}$ Note that we regard $l_{i}^{2}$, together with $l_{i} \cdot p_{j}$, as power 1 of $l_{i}$.
} 


\begin{tabular}{|c|c|c|c|c|c|}
\hline$\Gamma_{i}$ & & $\longrightarrow_{p_{L}}$ & & & \\
\hline$N_{(i)}$ & $\begin{array}{l}+N_{\mathrm{c}}\left(p_{2}, p_{1}, p_{L}, l_{3},\right. \\
\left.l_{2}, l_{1}-p_{L}\right)\end{array}$ & $\begin{array}{l}+N_{\mathrm{c}}\left(p_{L}, p_{1}, p_{2},\right. \\
\left.l_{1}-p_{L}, l_{2}, l_{3}\right)\end{array}$ & $\begin{array}{l}+N_{\mathrm{c}}\left(p_{1}, p_{2}, p_{3}, l_{1},\right. \\
\left.l_{2}, l_{3}-p_{3}\right)\end{array}$ & $\begin{array}{l}+N_{\mathrm{c}}\left(p_{3}, p_{2}, p_{1},\right. \\
\left.l_{3}-p_{3}, l_{2}, l_{1}\right)\end{array}$ & $N_{\mathrm{a}}=s_{12}^{2}$ \\
\hline
\end{tabular}

Table 4. Non-vanishing diagrams $\Gamma$ and their numerators under the planar cut to determine remaining parameters. Note that there are two cuts from the last ladder diagram. The signs from color factors have been absorbed into the numerators $N_{(i)}$, and loop momenta can be assigned according to the ordering in (6.21).

three-particle triple cut. In this triple-cut channel, only missing terms are terms proportional to $l_{k}^{2}$.

By means of dual Jacobi relations, all other numerators can be obtained.

\section{Symmetry of numerators.}

We impose the condition that the numerator should have the symmetry inherited from the corresponding topology. For range-2 diagrams, the numerators have no free parameter and they also automatically satisfy the symmetry constraint.

For the range-3 case, the symmetries of topologies in figure 12(d) and figure 12(e) are similar to the symmetries of tree-level amplitudes. For example, for topology figure $12(\mathrm{~d})$ we have the condition similar to $(6.11)$

$$
N_{\mathrm{d}}\left(p_{1}, p_{2}, p_{3}, l_{1}, l_{2}, l_{3}\right)=(-1)^{\sigma} N_{\mathrm{d}}\left(\sigma\left\{p_{1}, p_{2}, p_{3}\right\}, \sigma\left\{l_{1}, l_{2}, l_{3}\right\}\right), \quad \text { for } \forall \sigma \in S_{3} .
$$

The above symmetry constraints give three equations as

$$
2 c_{1}=1+2 c_{8}, \quad c_{2}+c_{3}=c_{7}+c_{9}, \quad c_{1}+c_{2}+c_{5}=c_{4}+c_{7}+c_{8} .
$$

5. Determining remaining parameters via unitarity.

Using the following unitarity cut channel|the planar two-particle triple-cut channel, we can further give more constraint on these parameters.

Concretely, we consider planar $s_{12}$ triple-cut (TC) channel as in figure 5(b). Basing on our discussion in section 4 , we act the loop correction with trivalent color factor on a single trace operator and expand the color factor of $\boldsymbol{F}^{(2)}=\boldsymbol{I}^{(2)} \boldsymbol{F}^{(0)}$ via trace basis. Our target color factor is $N_{c}^{2} \operatorname{tr}\left(a_{1} a_{2} \cdots a_{L}\right)$, and this cut channel can detect the following terms:

$$
\begin{aligned}
& \int \mathrm{dPS}_{3, l} \hat{\mathcal{F}}_{L+1}^{(0)}\left(-l_{1},-l_{2},-l_{3}, p_{3}, \ldots, p_{L}\right) \hat{\mathcal{A}}_{5}^{(0)}\left(l_{3}, l_{2}, l_{1}, p_{1}, p_{2}\right) \\
& =\left.\left[\left(\boldsymbol{I}_{12}^{(2)}+\boldsymbol{I}_{L 12}^{(2)}+\boldsymbol{I}_{123}^{(2)}\right) \operatorname{tr}\left(a_{1} a_{2} \cdots a_{L}\right)\right]_{N_{c}^{2} \operatorname{tr}\left(a_{1} a_{2} \cdots\right)}\right|_{s_{12}-\mathrm{TC}}=\left.\sum_{i=1}^{6} \frac{N_{(i)}}{D_{i}}\right|_{s_{12}-\mathrm{TC}},
\end{aligned}
$$

where diagrams contributing to this cut channel are listed in table 4. Using this cut constraint, one finds four more equations for these parameters:

$$
c_{2}=0, \quad c_{3}=0, \quad 0=-\frac{1}{2}+c_{1}, \quad \frac{1}{2}-c_{4}+c_{5}=0 .
$$


After using (6.20) and (6.22), there are still two parameters, chosen as $c_{4}, c_{6}$. It turns out that kinematic terms corresponding to these parameters do not change the final results of form factors. Below we will discuss the role of these parameters in more detail.

\section{Final results and discussion}

The final results for the range- 3 master numerator are

$$
\begin{aligned}
N_{\mathrm{c}}\left(p_{1}, p_{2}, p_{3}, l_{1}, l_{2}, l_{3}\right)= & s_{l_{2} l_{3}} s_{12}-\frac{1}{2} s_{l_{1} 12} s_{123}-\frac{1}{2} l_{2}^{2} s_{12} \\
& +\frac{1}{2} l_{1}^{2} s_{23}+l_{2}^{2}\left(c_{4} s_{13}+c_{6}\left(s_{12}+s_{23}\right)\right) .
\end{aligned}
$$

An interesting fact is that numerators $N_{j}$ with $j \in\{\mathrm{e}, \mathrm{f}, \mathrm{g}, \mathrm{h}, \mathrm{i}\}$ are not equal to zero, as required by the CK duality, but all of the them have actually zero contribution to the form factor.

First, any term proportional to $c_{4}$ or $c_{6}$ gives a scaleless integral that is zero in dimensional regularization. For example, the $l_{2}^{2}$ terms for $N_{\mathrm{c}}$ in $(6.23)$ correspond to massless bubbles; and similarly for those terms proportional to $c_{4,6}$ in $N_{j}$ with $j \in$ $\{\mathrm{e}, \mathrm{f}, \mathrm{g}, \mathrm{h}, \mathrm{i}\}\left(c_{4,6}\right.$ are cancelled in $\left.N_{\mathrm{b}}\right)$. If we set $c_{4}=c_{6}=0$, then $N_{\mathrm{i}}=0$ and the last diagram vanishes in figure 12. In the following discussion, for simplicity and without changing the form factor results, we present results with $c_{4,6}=0$.

Second, for the remaining integrals of topologies $j \in\{\mathrm{e}, \mathrm{f}, \mathrm{g}, \mathrm{h}\}$, they are also all equivalent to scaleless integrals. Alternatively, one may also argue the vanishing of $\{\mathrm{e}, \mathrm{g}, \mathrm{h}\}$ by using integral symmetries. For example, for $N_{\mathrm{e}}$, the integral is proportional to:

$$
\propto \int \mathrm{d}^{D} l_{2} \mathrm{~d}^{D} l_{3} \frac{\left(l_{3}^{2}-l_{2}^{2}\right)}{l_{1}^{2} l_{2}^{2} l_{3}^{2}\left(l_{2}+l_{3}\right)^{2}\left(l_{1}+p_{1}\right)^{2}\left(l_{1}+p_{1}+p_{2}\right)^{2}}=0,
$$

which is zero because the numerator $N_{\mathrm{e}}$ is anti-symmetric when exchanging $l_{2}$ and $l_{3}$ but the denominator and measure does not change under this permutation. The vanishing of topology $\Gamma_{\mathrm{f}}$, however, relies solely on its equivalence to a scaleless integral.

To summarize, the full-color two-loop correction, as given in (3.34), reads

$$
\begin{aligned}
& \boldsymbol{I}_{123}^{(2)}=\sum_{\sigma \in S_{3} \times S_{3}} \sum_{i=\mathrm{c}}^{\mathrm{h}} \int \prod_{j=1}^{2} \frac{\mathrm{d}^{D} l_{i}}{i(\pi)^{\frac{D}{2}}} \frac{1}{S_{i}^{123}} \sigma \cdot \frac{\check{\mathcal{C}}_{i}^{123} N_{i}^{123}}{\prod_{a} d_{i, a}}, \\
& \boldsymbol{I}_{12}^{(2)}=\sum_{\sigma \in S_{2} \times S_{2}} \sum_{i=\mathrm{a}}^{\mathrm{b}} \int \prod_{j=1}^{2} \frac{\mathrm{d}^{D} l_{i}}{i(\pi)^{\frac{D}{2}}} \frac{1}{S_{i}^{12}} \sigma \cdot \frac{\check{\mathcal{C}}_{i}^{12} N_{i}^{12}}{\prod_{a} d_{i, a}},
\end{aligned}
$$

where we summarize the trivalent topology in figure 12 and the corresponding factors in table 5 . Note that the permutations here are fully permuting both internal and external fields, i.e. full permutations of $\left\{p_{1}, p_{2}, p_{3}\right\}$ and $\left\{l_{1}, l_{2}, l_{3}\right\}$, considering both internal and external fields are identical. 


\begin{tabular}{|l|c|l|}
\hline$\Gamma_{i}$ & $N_{i}\left(\right.$ with $\left.c_{4}=c_{6}=0\right)$ & $S_{i}$ \\
\hline$(\mathrm{a})$ & $s_{12}^{2}$ & 2 \\
\hline$(\mathrm{b})$ & $s_{12}^{2}$ & 4 \\
\hline$(\mathrm{c})$ & $s_{12} s_{l_{2} l_{3}}-\frac{1}{2} s_{123} s_{12 l_{1}}-\frac{1}{2} l_{2}^{2} s_{12}+\frac{1}{2} l_{1}^{2} s_{23}$ & 1 \\
\hline$(\mathrm{d})$ & $\frac{1}{2} s_{12}\left[\left(s_{2 l_{2}}-s_{1 l_{1}}\right)+2 p_{3} \cdot\left(l_{2}-l_{1}\right)\right]+$ cyc. & 6 \\
\hline$(\mathrm{e})$ & $\frac{1}{2} s_{12}\left(l_{2}^{2}-l_{3}^{2}\right)$ & 2 \\
\hline (f) & $\frac{1}{2}\left(s_{13}-s_{12}\right) l_{1}^{2}$ & 2 \\
\hline (g) & $\frac{1}{2}\left(s_{12}-s_{13}\right)\left(l_{2}^{2}-l_{3}^{2}\right)$ & 2 \\
\hline$(\mathrm{h})$ & $N_{g}$ & 4 \\
\hline
\end{tabular}

Table 5. Various coefficients for the CK-dual BPS form factor. Trivalent topologies $\Gamma_{i}$ are given in figure 12. Color factors $\mathcal{C}_{i}$ can be directly read out from diagrams, given the rule that each vertex gives a $f^{a b c}$ with legs $a, b, c$ arranged in clockwise order. Note that the last topology (e) actually has zero contribution, we keep it here in order to manifest the CK duality of the numerators.

Last but not least, let us compare the above result obtained using CK duality with the unitarity result in section 5.2. An obvious difference is that for range- 2 densities, the CK result in (6.25) contains only ladder integrals while in (5.35a) and (5.35b) there is also another type of integral

$$
\mathcal{I}^{\prime}(12) \equiv>_{p_{p_{2}}}^{p_{1}}
$$

Calculation of the difference between the CK and the unitarity results shows that the difference $\Delta \boldsymbol{I} \equiv \boldsymbol{I}^{\mathrm{CK}}-\boldsymbol{I}^{\text {unitarity }}$ can be given as: ${ }^{16}$

$$
\begin{aligned}
& \Delta \boldsymbol{I}=\sum_{i<j<k}\left\{\sum_{\substack{\sigma^{i} \in \mathbb{Z}_{3} \\
\sigma^{e} \in S_{3}}} \check{\mathcal{D}}_{\Delta_{1}}\left(\sigma^{e}, \sigma^{i}\right) \sigma^{e} \cdot\left(\mathcal{I}^{\prime}(i j)+\mathcal{I}^{\prime}(j k)\right)\right. \\
& \left.-\sum_{\sigma^{e} \in S_{3}} \check{\mathcal{D}}_{\Delta_{2}}\left(\sigma^{e}\right)\left(\mathcal{I}^{\prime}(i j)+\mathcal{I}^{\prime}(j k)+\mathcal{I}^{\prime}(i k)\right)\right\} \\
& +2 \sum_{i<j} \sum_{\sigma^{e} \in S_{2}}\left(\check{\mathcal{D}}_{\Delta_{3}}\left(\sigma^{e}\right)-2 \check{\mathcal{D}}_{\Delta_{4}}(\mathbf{1})\right) \mathcal{I}^{\prime}(i j)
\end{aligned}
$$

\footnotetext{
${ }^{16}$ To get this result, one may need to expand CK results on the trivalent basis in section 5.1 using the equations like (A.2) in appendix A. Note that $N_{\mathrm{d}}$ is actually equivalent to the one we obtained in section 5.2.
} 
where $\mathcal{I}^{\prime}(i j)$ is the above mentioned range- 2 function and origins from a redistribution between range- 2 and range- 3 densities:

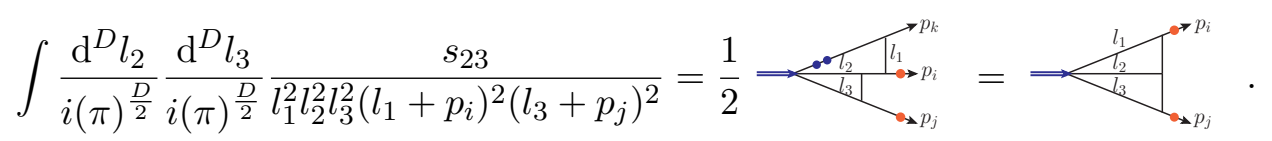

One can check that

$$
\Delta \boldsymbol{I} \cdot \boldsymbol{F}^{(0)}=0,
$$

with arbitrary $\boldsymbol{F}^{(0)}$, regardless of the length and color structure of corresponding (BPS) operator. In conclusion, we see that the two forms obtained in this section and the last section are equivalent. Similar identities will also be used in next section when we check the IR divergence cancellation.

\section{IR, UV divergences and finite remainder}

Having obtained the full-color form factor integrands in the last two sections, one can study the properties of integrated results. The bare form factors contain infrared (IR) and ultraviolet (UV) divergences. Since we consider the full-color results, both IR and UV structures depend on the color structure. The similarity and difference between planar and non-planar results will be a main focus of our discussion in this section.

As a brief outline, we will first review the planar IR structure and then generalize it to the full-color case in section 7.1. Second, we discuss the finite remainder functions of the BPS form factor in section 7.2, where we find a new non-planar contribution to the maximally transcendentality part. Third, we consider the SU(2) non-BPS form factor section 7.3, with an emphasis on the new feature of UV divergences and the non-planar dilatation operator. In the end, we highlight those new non-planar features and discuss their origin. Some technical details are given in appendix F.

\subsection{Infrared structure with full-color}

Infrared divergences originate from integral configurations where loop momenta become soft or collinear. Such singularities are universal in the sense that they only depend on the external on-shell particles and are irrelevant to the type of operators in the form factors. Since the BPS form factor contains no UV divergences, it provides a clean object to understand the IR structure, which we focus on in this subsection.

In the planar limit, the color structure is trivial and IR divergences can be simply captured by the BDS ansatz part [87], introduced originally for planar $\mathcal{N}=4$ amplitudes. On the other hand, for full-color form factors, the color mixing effect generates new interesting structure. In particular, there are new IR contributions at $\mathcal{O}(1 / \epsilon)$ order which involve three external legs at the same time. Such terms are the so-called $\boldsymbol{H}^{(2)}$ functions first obtained in the four-point amplitudes $[88,89]$ and then derived for general $n$-point amplitudes in [90]. They satisfy the color-dipole formula which determines the complete IR singularities up to two loops $[61,62]$. Our computation of $n$-point form factors will provide 
an independent concrete check for the dipole formula of amplitudes and form factors that involve arbitrary number of external legs.

Below we first briefly review the planar IR structure and then consider the generalization to full-color dependence.

Planar IR structure and BDS ansatz formula. At planar level, the IR structure has a simple form because all the Feynman diagrams are planar so that all the internal lines are confined to the wedges formed between two adjacent hard lines. As a result, planar IR divergences can be simply captured by the two-point Sudakov form factor [91-93]. Analysis via renormalization group shows that IR divergence can be given as a simple exponential form $[87,94]$ :

$$
\log \underline{\mathcal{I}}=-\sum_{l=1}^{\infty} g^{2 l}\left[\frac{\gamma_{\text {cusp }}^{(l)}}{(2 l \epsilon)^{2}}+\frac{\mathcal{G}_{\text {coll }}^{(l)}}{2 l \epsilon}\right] \sum_{i=1}^{n}\left(-s_{i i+1}\right)^{-l \epsilon} \cdot \mathbf{1}+\mathcal{O}\left(\epsilon^{0}\right),
$$

where $\underline{\mathcal{I}}^{(l)}$ is the l-loop renormalized planar loop correction (for BPS form factor, $\mathcal{I}=\mathcal{I}$ ), $\gamma_{\text {cusp }}$ is cusp anomalous dimension $[95,96]$ and $\mathcal{G}_{\text {coll }}$ is collinear anomalous dimension [97, 98]. ${ }^{17}$ Note that the trivial $N_{\mathrm{c}}$ dependence are included in $g^{2 l}$; for the non-planar case, we will use $g_{\mathrm{YM}}$ rather than $g$ and explicitly write down the $N_{\mathrm{c}}$ dependence.

It is convenient to rewrite (7.1) as the BDS form [87], and at two-loop level:

$$
\underline{\mathcal{I}}^{(2)}=\frac{1}{2}\left(\underline{\mathcal{I}}^{(1)}(\epsilon)\right)^{2}+f^{(2)}(\epsilon) \underline{\mathcal{I}}^{(1)}(2 \epsilon)+\mathcal{R}^{(2)}+\mathcal{O}(\epsilon),
$$

where the divergences are all captured in the first two terms and are determined by the one-loop correction $\mathcal{I}^{(1)}$, together with a universal kinematic independent quantity

$$
f^{(2)}(\epsilon)=-2 \zeta_{2}-2 \zeta_{3} \epsilon-2 \zeta_{4} \epsilon^{2},
$$

which contains the information of two-loop cusp and collinear anomalous dimensions. The remaining function $\mathcal{R}^{(2)}$ is two-loop finite remainder function.

As a pure side remark, one may compare the BDS subtraction with the Catani IR subtraction [103] (see also [104]):

$$
\begin{aligned}
& \mathcal{F}^{(1)}=I^{(1)}(\epsilon) \mathcal{F}^{(0)}+\mathcal{F}^{(1), \text { fin }}+\mathcal{O}(\epsilon), \\
& \mathcal{F}^{(2)}=I^{(2)}(\epsilon) \mathcal{F}^{(0)}+I^{(1)}(\epsilon) \mathcal{F}^{(1)}+\mathcal{F}^{(2), \text { fin }}+\mathcal{O}(\epsilon),
\end{aligned}
$$

where

$$
\begin{aligned}
& I^{(1)}(\epsilon)=-\frac{e^{\gamma_{E} \epsilon}}{\Gamma(1-\epsilon)} \frac{1}{\epsilon^{2}} \sum_{i=1}^{n}\left(-\frac{s_{i, i+1}}{\mu^{2}}\right)^{-\epsilon} \\
& I^{(2)}(\epsilon)=-\frac{1}{2}\left[I^{(1)}(\epsilon)\right]^{2}-\frac{e^{-\gamma_{E} \epsilon} \Gamma(1-2 \epsilon)}{\Gamma(1-\epsilon)} \frac{\pi^{2}}{3} I^{(1)}(2 \epsilon)+n \frac{e^{\gamma_{E} \epsilon}}{\epsilon \Gamma(1-\epsilon)} \frac{\zeta_{3}}{2} .
\end{aligned}
$$

\footnotetext{
${ }^{17}$ The non-planar corrections of cusp and collinear anomalous dimensions only start at fourth loop order. The analytic expression of the non-planar $\mathcal{N}=4$ cups anomalous dimension were obtained based on both Wilson loop [99] and form factor [100, 101] computations. The $\mathcal{N}=4$ non-planar collinear anomalous dimension is so far only known numerically [102].
} 
Although the original Catani subtraction is used in QCD, it can be applied here to $\mathcal{N}=4$ SYM theory by simply dropping lower transcendental pieces (including the beta function contribution). Note that BDS and Catani subtractions are equivalent for the divergence parts, but they have a (scheme-changing) difference in the finite remainders.

Non-planar IR structure and dipole formula. By dressing color factors, a generalization of $(7.2)$ is $[88-90]$

$$
\underline{\boldsymbol{I}}^{(2)}=\frac{1}{2}\left(\underline{\boldsymbol{I}}^{(1)}(\epsilon)\right)^{2}+\tilde{f}^{(2)}(\epsilon) \underline{\boldsymbol{I}}^{(1)}(2 \epsilon)+\boldsymbol{H}^{(2)}(\epsilon)+\boldsymbol{R}^{(2)}+\mathcal{O}(\epsilon),
$$

which contains a new function $\boldsymbol{H}^{(2)}$ with only simple pole in $\epsilon$. In the planar limit, (7.8) should reproduce the planar IR structure (7.1), therefore, the new extra contribution $\boldsymbol{H}^{(2)}$ should be a pure non-planar effect. This form can be understood based on the dipoleformula (see [61, 62] for further discussion):

$$
\Gamma_{n}^{\text {dip. }}\left(\left\{p_{i}\right\}, \mu\right)=-\frac{1}{2} \gamma_{\text {cusp }}\left(g_{\mathrm{YM}} ; N_{\mathrm{c}}\right) \sum_{i<j} \log \left(\frac{-s_{i j}}{\mu^{2}}\right) \hat{\mathbf{T}}_{i} \cdot \hat{\mathbf{T}}_{j}+\sum_{i=1}^{n} \mathcal{G}_{\mathrm{coll}, i}\left(g_{\mathrm{YM}} ; N_{\mathrm{c}}\right) .
$$

Note that the coupling constant before $\boldsymbol{I}^{(2)}$ should be $\tilde{g}^{4}$ rather than $g^{4}$ (see also (3.3)):

$$
\tilde{g}^{2}=\frac{g_{\mathrm{YM}}^{2}}{(4 \pi)^{2}}\left(4 \pi e^{-\gamma_{\mathrm{E}}}\right)^{\epsilon}, \quad \tilde{g}^{2}=g^{2} / N_{c}
$$

Correspondingly, $\tilde{f}^{(2)}=N_{\mathrm{c}} f^{(2)}$ where the $N_{\mathrm{c}}$ factor comes from $g^{2} / \tilde{g}^{2}$. Below we justify this formula and derive the explicit form of $\boldsymbol{H}^{(2)}$ using the BPS form factor results.

Verification of IR structure. To perform IR subtraction as in (7.8), one needs to compute the square of full-color one-loop correction $\boldsymbol{I}^{(1)}=\sum_{i_{1}<i_{2}} \boldsymbol{I}_{i_{1} i_{2}}^{(1)}$ which is reviewed in appendix D. The one-loop square has the following structure

$$
\left(\boldsymbol{I}^{(1)}\right)^{2}=\sum_{i_{1}<i_{2}, i_{3}<i_{4}} \boldsymbol{I}_{i_{1} i_{2}}^{(1)} \boldsymbol{I}_{i_{3} i_{4}}^{(1)}=\left.\left(\boldsymbol{I}^{(1)}\right)^{2}\right|_{\text {range-2 }}+\left.\left(\boldsymbol{I}^{(1)}\right)^{2}\right|_{\text {range-3 }}+\left.\left(\boldsymbol{I}^{(1)}\right)^{2}\right|_{\text {range-4 }},
$$

where the range is defined in terms of length of $\left\{i_{1}, i_{2}\right\} \cup\left\{i_{3}, i_{4}\right\}$. For example:

$$
\left.\left(\boldsymbol{I}^{(1)}\right)^{2}\right|_{\text {range-2 }}=\sum_{i_{1}<i_{2}}\left(\boldsymbol{I}^{(1)} \boldsymbol{I}^{(1)}\right)_{i_{1} i_{2}},\left.\quad\left(\boldsymbol{I}^{(1)}\right)^{2}\right|_{\text {range-3 }}=\sum_{i_{1}<i_{2}<i_{3}}\left(\boldsymbol{I}^{(1)} \boldsymbol{I}^{(1)}\right)_{i_{1} i_{2} i_{3}} .
$$

The color factor of range- 2 and range- 3 interactions here can also be expanded on the trivalent basis of loop correction densities, i.e. $\check{\mathcal{D}}_{j}$ defined in (5.4)-(5.7). Although the detailed definition of one-loop square is a little bit subtle which will be clarified in appendix F.1, the final result is clear and simple:

- Range-2:

$$
\begin{aligned}
\left(\boldsymbol{I}^{(1)} \boldsymbol{I}^{(1)}\right)_{12} & =\sum_{j=25}^{27} \check{\mathcal{D}}_{j}\left[\left(\mathcal{I}^{(1)} \mathcal{I}^{(1)}\right)\left(p_{1}, p_{2}\right)\right]_{\check{\mathcal{D}}_{j}}, \\
{\left[\left(\mathcal{I}^{(1)} \mathcal{I}^{(1)}\right)\left(p_{1}, p_{2}\right)\right]_{\check{\mathcal{D}}_{25}} } & =\mathcal{I}^{(1)}(12)^{2}, \\
{\left[\left(\mathcal{I}^{(1)} \mathcal{I}^{(1)}\right)\left(p_{1}, p_{2}\right)\right]_{\check{\mathcal{D}}_{27}} } & =0 .
\end{aligned}
$$


- Range-3:

$$
\begin{aligned}
\left(\boldsymbol{I}^{(1)} \boldsymbol{I}^{(1)}\right)_{123} & =\sum_{j=1}^{24} \check{\mathcal{D}}_{j}\left[\left(\mathcal{I}^{(1)} \mathcal{I}^{(1)}\right)\left(p_{1}, p_{2}, p_{3}\right)\right]_{\check{\mathcal{D}}_{j}}, \\
{\left[\left(\mathcal{I}^{(1)} \mathcal{I}^{(1)}\right)\left(p_{1}, p_{2}, p_{3}\right)\right]_{\check{\mathcal{D}}_{1}} } & =2 \mathcal{I}^{(1)}(12) \mathcal{I}^{(1)}(23), \\
{\left[\left(\mathcal{I}^{(1)} \mathcal{I}^{(1)}\right)\left(p_{1}, p_{2}, p_{3}\right)\right]_{\check{\mathcal{D}}_{19}} } & =-\mathcal{I}^{(1)}(12) \mathcal{I}^{(1)}(23)-\mathcal{I}^{(1)}(23) \mathcal{I}^{(1)}(13)-\mathcal{I}^{(1)}(13) \mathcal{I}^{(1)}(12) .
\end{aligned}
$$

Note that the range-4 part is straightforward to write down and we will not give them here. Also, in the context of BPS form factors $\mathcal{I}^{(1)}(i j)=-\mathrm{I}_{\text {tri }}\left[s_{i j}\right]$ with $\mathrm{I}_{\text {tri }}$ defined in appendix D.

Using two-loop form factor results (5.35a)-(5.36b), and the expression for one-loopsquare (7.11), we can now apply (7.8) to check the cancellation of IR singularity. ${ }^{18}$ The dipole formula implies that the divergent part of the following quantity

$$
\underline{\boldsymbol{I}}^{(2)}-\frac{1}{2}\left(\underline{\boldsymbol{I}}^{(1)}(\epsilon)\right)^{2}-\tilde{f}^{(2)}(\epsilon) \underline{\boldsymbol{I}}^{(1)}(2 \epsilon)
$$

is expected to have $\epsilon$ simple pole only, which we will verify by direct calculation.

The remaining $1 / \epsilon$ divergent terms are summarized in the function $\boldsymbol{H}^{(2)}$, whose kinematic dependence is only polynomial of $\log s_{i j}$. More precisely, we find the following form:

$$
\begin{aligned}
\boldsymbol{H}^{(2)} & =\sum_{i<j<k} \boldsymbol{H}_{i j k}^{(2)}, \\
\boldsymbol{H}_{123}^{(2)} & =\left(\sum_{\sigma \in S_{3}}(-1)^{\sigma} \check{\mathcal{D}}_{\Delta_{2}}(\sigma)\right) \frac{1}{4 \epsilon} \log \left(\frac{-s_{12}}{-s_{23}}\right) \log \left(\frac{-s_{23}}{-s_{13}}\right) \log \left(\frac{-s_{13}}{-s_{12}}\right),
\end{aligned}
$$

where $\sigma$ represents permutation of $p_{1}, p_{2}, p_{3}$, and these permutations do not change the triple-log term up to a sign. Note that these terms have only components on the nonplanar set of color factors $\check{\mathcal{D}}_{\Delta_{2}}(\sigma)$. This is equivalent to the known result, see e.g. [86]. More details of the cancellation and derivation are given in appendix F.1, and we will provide an understanding of the $\boldsymbol{H}^{(2)}$ functions in section 7.4.

\subsection{BPS remainder}

After subtracting IR divergences, one obtains the finite remainder functions of full-color BPS form factors. As we will see, the non-planar BPS remainder receives a new contribution in the maximal transcendentality part.

A brief review of planar remainder. We first review the planar remainder, which is discussed in detail in [14]. Planar remainder can be expressed as summing remainder density functions as

$$
\mathcal{R}^{\mathrm{PL}}=\sum_{i} \mathcal{R}_{i(i+1)(i+2)}^{\mathrm{PL}}
$$

\footnotetext{
${ }^{18}$ The computation of the integrated result is standard: one can first perform IBP reduction for the integrand using public packages, (see e.g. [105-107]), and the master integrals in our problem are all known in terms of $2 \mathrm{~d}$ harmonic polylogarithms [108, 109].
} 
and we recall the explicit form of the remainder here:

$$
\mathcal{R}_{123}^{\mathrm{PL}}=\mathcal{R}_{\text {basis }}(u, v, w)+(u \leftrightarrow v)+\zeta_{4},
$$

where

$$
u=\frac{s_{12}}{s_{123}}, \quad v=\frac{s_{23}}{s_{123}}, \quad w=\frac{s_{13}}{s_{123}},
$$

and $\mathcal{R}_{\text {basis }}(u, v, w)$ is

$$
\begin{aligned}
G(\{1 & -u, 1-u, 1,0\}, v)-\operatorname{Li}_{4}(1-u)-\operatorname{Li}_{4}(u)+\operatorname{Li}_{4}\left(\frac{u-1}{u}\right) \\
& -\log \left(\frac{1-u}{w}\right)\left[\operatorname{Li}_{3}\left(\frac{u-1}{u}\right)-\operatorname{Li}_{3}(1-u)\right] \\
& -\log (u)\left[\operatorname{Li}_{3}\left(\frac{v}{1-u}\right)+\operatorname{Li}_{3}\left(-\frac{w}{v}\right)+\operatorname{Li}_{3}\left(\frac{v-1}{v}\right)\right] \\
& -\operatorname{Li}_{2}\left(\frac{u-1}{u}\right) \operatorname{Li}_{2}\left(\frac{v}{1-u}\right)+\operatorname{Li}_{2}(u)\left[\log \left(\frac{1-u}{w}\right) \log (v)+\frac{1}{2} \log ^{2}\left(\frac{1-u}{w}\right)\right] \\
& +\frac{1}{24} \log ^{4}(u)-\frac{1}{6} \log ^{3}(u) \log (w)+\frac{1}{3} \log (u) \log ^{3}(v)+\frac{1}{3} \log (u) \log ^{3}(1-u) \\
& -\frac{1}{8} \log ^{2}(u) \log ^{2}(v)-\frac{1}{2} \log ^{2}(1-u) \log (u) \log \left(\frac{w}{v}\right)-\frac{1}{2} \log (1-u) \log ^{2}(u) \log (v) \\
& -\zeta_{2}\left[\log (u) \log \left(\frac{1-v}{v}\right)+\frac{1}{2} \log ^{2}\left(\frac{1-u}{w}\right)-\frac{1}{2} \log ^{2}(u)\right]+\zeta_{3} \log (u),
\end{aligned}
$$

where $G$ is a Goncharov polylogarithm.

To get this compact expression, symbol (denoted as $\mathcal{S}$ ) is a powerful tool, see [110, 111]. Here we only briefly review the strategy of simplifying remainders with the aid of symbols below.

1. To start with, one can determine terms that can not be expressed with classical polylogarithms. If a transcendentality-4 function $f$ satisfies Goncharov criterion [110]

$$
\left.\delta \circ \mathcal{S}(f)\right|_{\Lambda^{2} B_{2}} \equiv \mathcal{S}_{a b c d}-\mathcal{S}_{b a c d}-\mathcal{S}_{a b d c}+\mathcal{S}_{b a d c}-(a \leftrightarrow c, b \leftrightarrow d)=0,
$$

then it can be re-written in terms of classical polylogarithms, i.e. $\log (x), \operatorname{Li}_{k}(x)$. Planar remainder density $\mathcal{R}_{123}^{\mathrm{PL}}$ does not pass this criterion and one can use $G(\{1-u, 1-$ $u, 1,0\}, v)$ to capture terms beyond classical polylogarithms, see [14] for more details.

2. In order to determine the classical polylogrithm part, one can use an ansatz as linear combination of all possible polynomial of classical polylogarithms with transcendentality-4 and solve the ansatz at symbol level.

3. Finally, one can fix the terms ignored by symbols, which are linear combination of functions $\zeta_{2} \log (x) \log (y), \zeta_{2} \operatorname{Li}_{2}(x), \zeta_{3} \log (x)$ and $\zeta_{4}$ in transcendentality-4 case, for example numerically.

To compare with the non-planar remainders discussed later, we comment on the definition of remainder at density level. In the planar case, one can sum up range- 2 and range- 3 
densities to define remainder density in (7.17) as

$$
\begin{aligned}
\mathcal{R}_{123}^{\mathrm{PL}}= & \mathcal{I}_{123}^{(2), \mathrm{PL}}+\frac{1}{2}\left(\mathcal{I}_{12}^{(2), \mathrm{PL}}+\mathcal{I}_{23}^{(2), \mathrm{PL}}\right)-\frac{1}{2}\left[\frac{1}{2}\left(\mathcal{I}_{12}^{(1), \mathrm{PL}}\right)^{2}+\frac{1}{2}\left(\mathcal{I}_{23}^{(1), \mathrm{PL}}\right)^{2}+2 \mathcal{I}_{12}^{(1), \mathrm{PL}} \mathcal{I}_{23}^{(1), \mathrm{PL}}\right] \\
& -\left.\tilde{f}^{(2)}(\epsilon)\left(\mathcal{I}_{12}^{(1), \mathrm{PL}}+\mathcal{I}_{23}^{(1), \mathrm{PL}}\right)\right|_{\epsilon \rightarrow 2 \epsilon},
\end{aligned}
$$

which means (planar) IR subtraction (7.2) is true at density level.

Full-color remainder densities. For the non-planar case, in contrast, (7.8) may not hold directly at density level. Below we would like to introduce the density of remainders for range- 3 and range- 2 separately. To achieve this, we need to introduce some auxiliary divergent quantities which appear in remainder densities. The auxiliary quantities will be shown to cancel in the full form factor, after summing over all densities. Such a definition, unlike planar case (7.22) adding range-3 and range-2 together, will make it easier to deal with the non-planar contribution and also help to understand the structure better.

For the convenience of discussion below, we introduce $\mathcal{I}_{\mathrm{BPS}, \mathrm{a}}^{(2)}\left(p_{1}, p_{2}, p_{3}\right)$, or denoted as $\mathcal{I}_{\mathrm{BPS}, \mathrm{a}}^{(2)}(123)$, to represent the terms in parenthesis in (5.40) or (5.41):

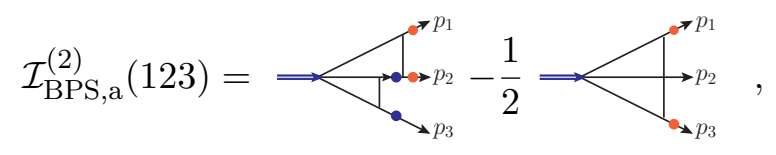

as well as the following ones for range-2:

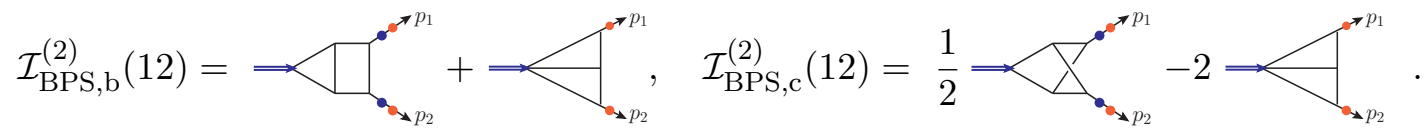

We first give a definition for the (range-3) color-stripped remainder density associated with color factor $\check{\mathcal{D}}_{1}$. It is helpful to consider first the divergent part. Following (7.15), we find that

$$
\left(\left[\mathcal{I}^{(2)}(123)\right]_{\check{\mathcal{D}}_{1}}-\frac{1}{2}\left[\left(\mathcal{I}^{(1)} \mathcal{I}^{(1)}\right)(123)\right]_{\check{\mathcal{D}}_{1}}\right)_{\mathrm{div}}=U(12)+U(23)
$$

where $U(i j)$ are the auxiliary divergences mentioned above, and are defined as

$$
U(i j)=\left(-\frac{\zeta_{2}}{2 \epsilon^{2}}-\frac{2 \zeta_{3}}{\epsilon}\right)\left(-s_{i j}\right)^{-2 \epsilon} .
$$

Thus, the corresponding remainder density can be defined as

$$
\left[\mathcal{R}^{(2)}(123)\right]_{\check{\mathcal{D}}_{1}}=\left(\mathcal{I}_{\mathrm{BPS}, \mathrm{a}}^{(2)}(123)-\frac{1}{2} \mathcal{I}^{(1)}(12) \mathcal{I}^{(1)}(23)-U(12)\right)+\left(p_{1} \leftrightarrow p_{3}\right),
$$

where we have used explicit expression in (5.41), (7.14) and (7.26).

Similarly, the range-2 density remainder associated with $\check{\mathcal{D}}_{25}$ can be defined as

$$
\left[\mathcal{R}^{(2)}(12)\right]_{\check{\mathcal{D}}_{25}}=\mathcal{I}_{\mathrm{BPS}, \mathrm{b}}^{(2)}(12)-\frac{1}{2}\left(\mathcal{I}^{(1)}(12)\right)^{2}-(-2) U(12)-f^{(2)}(\epsilon)\left(\mathcal{I}^{(1)}(12)\right)_{\epsilon \rightarrow 2 \epsilon},
$$

and it is easy to check that $\left[\mathcal{R}^{(2)}(12)\right]_{\check{\mathcal{D}}_{25}}=19 \zeta_{4} / 2$. 
Note that the above remainder densities in (7.27) and (7.28) are different from planar definition $\mathcal{R}_{123}^{\mathrm{PL}}$ in (7.22), since the latter is a combination of range- 3 and range- 2 densities. One can relate $\mathcal{R}_{123}^{\mathrm{PL}}$ to the densities in (7.27) and (7.28) by

$$
\begin{aligned}
\mathcal{R}_{123}^{\mathrm{PL}}= & {\left[\mathcal{R}^{(2)}(123)\right]_{\check{\mathcal{D}}_{1}}+\frac{1}{2}\left[\mathcal{R}^{(2)}(12)\right]_{\check{\mathcal{D}}_{25}}+\frac{1}{2}\left[\mathcal{R}^{(2)}(23)\right]_{\check{\mathcal{D}}_{25}} } \\
= & {\left[\mathcal{I}_{\mathrm{BPS}, \mathrm{a}}^{(2)}(123)+\frac{1}{2} \mathcal{I}_{\mathrm{BPS}, \mathrm{b}}^{(2)}(12)\right.} \\
& \left.-\frac{1}{2}\left(\mathcal{I}^{(1)}(12) \mathcal{I}^{(1)}(23)+\frac{1}{2}\left(\mathcal{I}^{(1)}(12)\right)^{2}\right)-\frac{1}{2} f^{(2)}(\epsilon)\left(\mathcal{I}^{(1)}(12)\right)_{\epsilon \rightarrow 2 \epsilon}\right]+\left(p_{1} \leftrightarrow p_{3}\right) .
\end{aligned}
$$

In this combination one can see clearly that the $U(12)$ and $U(23)$ cancel between range-2 and range-3. Also, it is straightforward to get

$$
\left[\mathcal{R}^{(2)}(123)\right]_{\check{\mathcal{D}}_{1}}=\mathcal{R}_{\text {basis }}(u, v, w)+(u \leftrightarrow v)+20 \zeta_{4} .
$$

Now we turn to the calculation of the remainder associated to non-planar color factors, i.e. $\left[\mathcal{R}^{(2)}\right]_{\check{\mathcal{D}}_{19}}$ and $\left[\mathcal{R}^{(2)}\right]_{\check{\mathcal{D}}_{27}}$. The range-3 non-planar remainder can be defined as:

$$
\begin{aligned}
{\left[\mathcal{R}^{(2)}(123)\right]_{\check{\mathcal{D}}_{19}}=} & \left(-\mathcal{I}_{\mathrm{BPS}, \mathrm{a}}^{(2)}(123)+\frac{1}{2} \mathcal{I}^{(1)}(12) \mathcal{I}^{(1)}(23)+U(12)\right)+\operatorname{cyc}\left(p_{1}, p_{2}, p_{3}\right) \\
& -\frac{1}{4 \epsilon} \log \left(\frac{-s_{12}}{-s_{23}}\right) \log \left(\frac{-s_{23}}{-s_{13}}\right) \log \left(\frac{-s_{13}}{-s_{12}}\right)
\end{aligned}
$$

where the last term in (7.31) comes precisely from $\boldsymbol{H}_{123}^{(2)}$ in (7.16), and to cancel the divergence we also add auxiliary function $U(i j)$, which will cancel in the end. The range-2 remainder is defined as

$$
\frac{1}{2}\left[\mathcal{R}^{(2)}(12)\right]_{\check{\mathcal{D}}_{27}}=\left[\mathcal{I}_{\mathrm{BPS}, \mathrm{c}}^{(2)}(12)-4 U(12)-f^{(2)}(\epsilon)\left(\mathcal{I}^{(1)}(12)\right)_{\epsilon \rightarrow 2 \epsilon}\right],
$$

and $\left[\mathcal{R}^{(2)}\right]_{\check{\mathcal{D}}_{27}}$ is also a pure number equals to $2 \times\left(-17 \zeta_{4}\right)$.

Now one may ask the question that: does the non-planar remainder have the same maximal transcendental part as the planar remainder? Interestingly, the answer is no. A simple calculation reveals that $\left[\mathcal{R}^{(2)}(123)\right]_{\check{\mathcal{D}}_{19}}$ can not be given as a linear combination of $\mathcal{R}_{\text {basis }}$ function in (7.20) plus pure numbers. To compute its analytic form, we follow the similar strategy as described above for the planar remainder.

We first check the Goncharov criterion (7.21). Observing that

$$
\left.\delta \circ \mathcal{S}\left(\left[\mathcal{R}^{(2)}\right]_{\check{\mathcal{D}}_{19}}\right)\right|_{\Lambda^{2} B_{2}}=-\left.\delta \circ(\mathcal{S}(G(\{1-u, 1-u, 1,0\}, v)+\operatorname{cyc}(u, v, w)))\right|_{\Lambda^{2} B_{2}},
$$

the non-classical part of $\left[\mathcal{R}^{(2)}\right]_{\check{\mathcal{D}}_{19}}$ can be chosen as the same $G$ functions as in $\mathcal{R}_{\text {basis }}$.

Inspired by this, we define $\mathrm{P}^{19}$

$$
\mathcal{R}^{\mathrm{NP}}=\left[\mathcal{R}^{(2)}\right]_{\check{\mathcal{D}}_{19}}+\mathcal{R}_{\text {basis }}(u, v, w)+\mathcal{R}_{\text {basis }}(v, w, u)+\mathcal{R}_{\text {basis }}(w, u, v)-3 \times \frac{19}{4} \zeta_{4},
$$

\footnotetext{
${ }^{19}$ The $\zeta_{4}$ term is separated intentionally to get symmetric properties of $\mathcal{R}_{123}^{\mathrm{NP}}$ in (7.35).
} 
as new non-planar (NP) information with the following symmetry property

$$
\mathcal{R}^{\mathrm{NP}}(u, v, w)=(-1)^{\sigma} \mathcal{R}^{\mathrm{NP}}(\sigma(u, v, w)) .
$$

Since $\mathcal{R}^{\mathrm{NP}}$ passes the Goncharov criterion and can be expressed as classic polylogarithm consequently. It can be fixed using the ansatz method aforementioned and the final expression takes a very simple form as

$$
\mathcal{R}^{\mathrm{NP}}(u, v, w)=\mathcal{R}_{\mathrm{basis}}^{\mathrm{NP}}(u, v, w)+\operatorname{cyc}(u, v, w),
$$

where we introduce another basis function

$$
\mathcal{R}_{\text {basis }}^{\mathrm{NP}}(u, v, w)=\operatorname{Li}_{3}\left(1-\frac{1}{u}\right) \log \left(\frac{v}{w}\right)+\frac{1}{12} \log (u)^{3} \log \left(\frac{v}{w}\right)+\zeta_{2} \log (1-u) \log \left(\frac{v}{w}\right) .
$$

As a result, the range-3 non-planar remainder density can be given as

$$
\left[\mathcal{R}^{(2)}(123)\right]_{\check{\mathcal{D}}_{19}}=\left[-\mathcal{R}_{\text {basis }}(u, v, w)+\mathcal{R}_{\text {basis }}^{\mathrm{NP}}(u, v, w)+\frac{19}{4} \zeta_{4}\right]+\operatorname{cyc}(u, v, w) .
$$

With the symmetry of (7.35), we can also dress color factor to $\mathcal{R}_{\mathrm{basis}}^{\mathrm{NP}}(u, v, w)$ and define

$$
\begin{aligned}
\boldsymbol{R}^{\mathrm{NP}} & =\sum_{i<j<k} \boldsymbol{R}_{i j k}^{\mathrm{NP}}, \\
\boldsymbol{R}_{123}^{\mathrm{NP}} & =\sum_{\sigma \in S_{3}} \check{\mathcal{D}}_{\Delta_{2}}(\sigma) \mathcal{R}_{\mathrm{basis}}^{\mathrm{NP}}(\sigma(u, v, w))=\left(\sum_{\sigma \in S_{3}}(-1)^{\sigma} \check{\mathcal{D}}_{\Delta_{2}}(\sigma)\right) \mathcal{R}_{\mathrm{basis}}^{\mathrm{NP}}(u, v, w) .
\end{aligned}
$$

This structure is similar to the $\boldsymbol{H}^{(2)}$ function in (7.16). We will discuss more on this new non-planar contribution in section 7.4.

\subsection{UV renormalization, dilatation operator and remainders}

For non-BPS form factors, after subtracting the universal IR divergences, the remaining divergences have an ultra-violent origin. The UV divergences should be cancelled by the renormalization constant, which can be used to determine the dilatation operator. We apply this procedure to the $\mathrm{SU}(2)$ form factors.

We generalize the renormalization procedure in (2.15). Up to two loops, one has

$$
\begin{aligned}
& \underline{\boldsymbol{I}}^{(1)}=\boldsymbol{I}^{(1)}+\boldsymbol{Z}^{(1)}, \\
& \underline{\boldsymbol{I}}^{(2)}=\boldsymbol{I}^{(2)}+\boldsymbol{Z}^{(2)}+\boldsymbol{I}^{(1)} \boldsymbol{Z}^{(1)},
\end{aligned}
$$

where $\boldsymbol{Z}$ is the renormalized constant and $\underline{\boldsymbol{I}}$ is the full-color renormalized loop correction: at one-loop level, $\underline{\boldsymbol{I}}^{(1)}$, together with $\boldsymbol{I}^{(1)}$ and $\boldsymbol{Z}^{(1)}$, are given in appendix D; at two-loop level, from (7.8), we also have

$$
\boldsymbol{I}^{(2)}=-\left(\boldsymbol{Z}^{(2)}+\boldsymbol{I}^{(1)} \boldsymbol{Z}^{(1)}\right)+\frac{1}{2}\left(\underline{\boldsymbol{I}}^{(1)}\right)^{2}+\boldsymbol{H}_{\mathrm{SU}(2)}^{(2)}+\tilde{f}^{(2)}(\epsilon)\left(\underline{\boldsymbol{I}}^{(1)}\right)_{\epsilon \rightarrow 2 \epsilon}+\boldsymbol{R}^{(2)}+\mathcal{O}(\epsilon),
$$

where $\boldsymbol{H}_{\mathrm{SU}(2)}^{(2)}$, with details given in section 7.4, is similar to the $\boldsymbol{H}^{(2)}$ terms in (7.8).

For non-BPS form factors, we stress that $\boldsymbol{Z}$ and $\boldsymbol{R}$ include new information, which require exploration and are one center topic of the following discussion. 
Structure of two-loop SU(2) loop corrections. The structure of $\boldsymbol{I}^{(1)}$ for the SU(2) form factor is clear. In (D.2), we can see that UV and IR structures can be separated easily| triangle integrals captures IR divergences and both $\boldsymbol{Z}^{(1)}$ and $\boldsymbol{R}^{(1)}$ are determined by the bubble part.

At two-loop order, such a trivial separation is in general not possible. However, for the $\mathrm{SU}(2)$ form factor, we find it is convenient to group $\boldsymbol{I}^{(2)}$ in three parts:

$$
\boldsymbol{I}^{(2)}=\boldsymbol{I}_{\mathrm{BPS}}^{(2)}+\boldsymbol{I}_{\text {fish }}^{(2)}+\boldsymbol{I}_{\mathrm{mix}}^{(2)},
$$

which manifests the separation of IR and UV divergences. Let us explain the decomposition of (7.42) in detail:

- $\boldsymbol{I}_{\mathrm{BPS}}^{(2)}$ is the BPS part as discussed in (5.53). These integrals, physically, can be regarded as containing IR divergence only. Besides, these are the terms contributing to the maximally transcendental part of remainders.

This BPS part satisfies

$$
\boldsymbol{I}_{\mathrm{BPS}}^{(2)}=\frac{1}{2}\left(\underline{\boldsymbol{I}}_{\mathrm{tri}}^{(1)}\right)^{2}+\tilde{f}^{(2)}(\epsilon)\left(\underline{\boldsymbol{I}}_{\mathrm{tri}}^{(1)}\right)_{\epsilon \rightarrow 2 \epsilon}+\boldsymbol{H}_{\mathrm{BPS}}^{(2)}+\boldsymbol{R}_{\mathrm{a}}^{(2)},
$$

where $\boldsymbol{I}_{\text {tri }}$ is the triangle integral part ${ }^{20}$ of one-loop correction (see appendix D) and $\boldsymbol{H}_{\mathrm{BPS}}^{(2)}$ has the same kinematic part as $\boldsymbol{H}^{(2)}$ in (7.16). $\boldsymbol{R}_{\mathrm{a}}^{(2)}$ is equal to the BPS remainder and provides all the maximally transcendental part. Such a subtraction has no difference from our discussion in the previous sections and we will skip this part.

- The second term $\boldsymbol{I}_{\text {fish }}^{(2)}$ is composed of two types of integrals:

$$
\mathcal{I}_{\mathrm{SU}(2), \mathrm{a}}^{(2)}\left(p_{1}, p_{2}, p_{3}\right)=\overbrace{p_{p_{1}}}^{p_{2}}, \mathcal{I}_{\mathrm{SU}(2), \mathrm{b}}^{(2)}\left(p_{1}, p_{2}\right)=\overbrace{p_{2}}^{p_{1}}
$$

These integrals are special because they do not have divergences in soft region, in other words, they have UV divergence only. Explicit form of these integrals is listed in (D.8). The renormalization constant can be fixed using the following equation

$$
\boldsymbol{I}_{\text {fish }}^{(2)}=-\boldsymbol{Z}^{(2)}-\boldsymbol{I}_{\mathrm{bub}}^{(1)} \boldsymbol{Z}^{(1)}+\frac{1}{2}\left(\underline{\boldsymbol{I}}_{\mathrm{bub}}^{(1)}\right)^{2}+\boldsymbol{R}_{\mathrm{b}}^{(2)} .
$$

- The remaining part of integrand contains

$$
\mathcal{I}_{\mathrm{SU}(2), \mathrm{c}}^{(2)}\left(p_{1}, p_{2}, p_{3}\right)=
$$

\footnotetext{
${ }^{20}$ Later we also use $\boldsymbol{I}_{\text {bub }}$ as bubble integral part and $\underline{\boldsymbol{I}}_{\text {bub }}$ as renormalized bubble part. Their corresponding kinematic parts are denoted by simply replacing $\boldsymbol{I}$ with $\mathcal{I}$.
} 
This part has both UV and IR divergences but are all canceled by one-loop data:

$$
\boldsymbol{I}_{\text {mix }}^{(2)}=-\boldsymbol{I}_{\text {tri }}^{(1)} \boldsymbol{Z}^{(1)}+\underline{\boldsymbol{I}}_{\text {tri }}^{(1)} \underline{\boldsymbol{I}}_{\text {bub }}^{(1)}+\tilde{f}^{(2)}(\epsilon)\left(\underline{\boldsymbol{I}}_{\mathrm{bub}}^{(1)}\right)_{\epsilon \rightarrow 2 \epsilon}+\boldsymbol{R}_{\mathrm{c}}^{(2)} .
$$

Here $\boldsymbol{R}_{\mathrm{c}}^{(2)}$, accompanied with $\boldsymbol{R}_{\mathrm{b}}^{(2)}$ above, represent the lower transcendentality parts of finite remainders, and will be discussed in detail below.

An important problem to consider first is the color structure of such a separation. Indeed, equations (7.43)-(7.45) can be expanded on DDM basis defined in section 5.1 and the subtraction procedure is similar to the previous sections. We will not reproduce the details for such calculation. Rather, it will be helpful to reorganize the results, especially the color factors, and manifest the structure of $\tilde{\boldsymbol{I}}^{(2)}=\boldsymbol{I}_{\mathrm{mix}}^{(2)}+\boldsymbol{I}_{\text {fish }}^{(2)}$ which is defined in (5.53) and decomposed in (7.42). Without loss of generality, we specify fields $Y X X$ for sites 123 and $p_{1}^{Y} p_{2}^{X} p_{3}^{X}$ as external states, and any other fields configuration can be obtained by acting permutations and relabeling. The density form of (7.44)-(7.45) can be written as

$$
\begin{aligned}
\tilde{\boldsymbol{I}}_{123, \mathrm{fish}}^{(2)} & =\check{\mathcal{C}}_{\mathrm{fish}}^{r=3} \mathcal{I}_{\mathrm{SU}(2), \mathrm{a}}^{(2)}\left(p_{1}, p_{2}, p_{3}\right)+\left(a_{2}, p_{2} \leftrightarrow a_{3}, p_{3}\right), \\
\tilde{\boldsymbol{I}}_{12, \mathrm{fish}}^{(2)} & =\check{\mathcal{C}}_{\mathrm{fish}}^{r=2} \mathcal{I}_{\mathrm{SU}(2), \mathrm{b}}^{(2)}\left(p_{1}, p_{2}\right), \\
\tilde{\boldsymbol{I}}_{123, \text { mix }}^{(2)} & =\check{\mathcal{C}}_{\text {mix,A } \mathrm{A}}^{r=3} \mathcal{I}_{\mathrm{SU}(2), \mathrm{c}}^{(2)}\left(p_{1}, p_{2}, p_{3}\right)+\check{\mathcal{C}}_{\text {mix } \mathrm{B}}^{r=3} \mathcal{I}_{\mathrm{SU}(2), \mathrm{c}}^{(2)}\left(p_{2}, p_{1}, p_{3}\right)+\left(a_{2}, p_{2} \leftrightarrow a_{3}, p_{3}\right), \\
\tilde{\boldsymbol{I}}_{12, \text { mix }}^{(2)} & =\check{\mathcal{C}}_{\text {mix }}^{r=2} \mathcal{I}_{\mathrm{SU}(2), \mathrm{d}}^{(2)}\left(p_{1}, p_{2}\right),
\end{aligned}
$$

where $\check{\mathcal{C}}$ are color factors defined in terms of trivalent graphs as

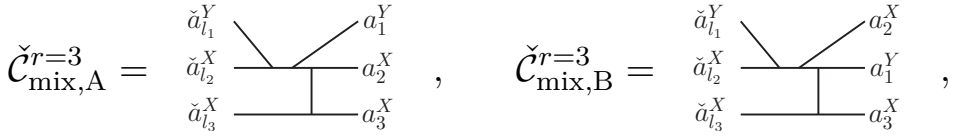

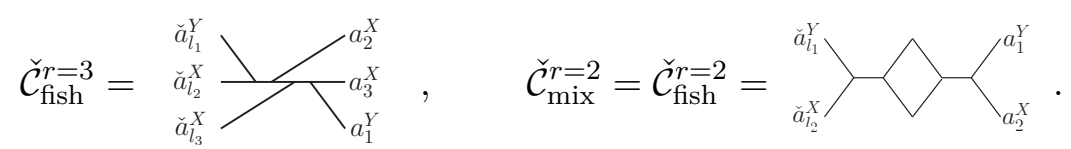

We will use the above form for the subtraction below and the complete derivation of this form can be found in appendix F.2. Technical details about the subsequent subtraction are also included there.

Renormalization and SU(2) dilatation operator. To obtain the two-loop renormalization constant and dilatation operator, one just needs to focus on (7.44). To realize that, we first check the structure of $\boldsymbol{Z}^{(1)} \boldsymbol{I}^{(1)}$

$$
\begin{aligned}
\left(\boldsymbol{Z}^{(1)} \boldsymbol{I}_{\text {bub }}^{(1)}\right)_{123} & =\check{\mathcal{C}}_{\text {fish }}^{r=3} \mathcal{Z}^{(1)} \mathcal{I}_{\text {bub }}^{(1)}(12)+\left(a_{2}, p_{2} \leftrightarrow a_{3}, p_{3}\right), \\
\left(\boldsymbol{Z}^{(1)} \boldsymbol{I}_{\text {bub }}^{(1)}\right)_{12} & =2 \stackrel{\check{\mathcal{C}}_{\text {fish }}^{r=2}}{\mathcal{Z}^{(1)}} \mathcal{I}_{\text {bub }}^{(1)}(12),
\end{aligned}
$$

where we have used the fact that $\mathcal{Z}^{(1)}$ have no kinematic dependence. The factor 2 can be explained as permuting the two intermediate fields in $\check{\mathcal{C}}_{\text {fish }}^{r=2} \mid$ they can be $X Y$ or $Y X$. This 
fact is easy to understand given the fact that the color factor of both $\boldsymbol{Z}^{(1)}$ and $\boldsymbol{I}_{\text {bub }}^{(1)}$ is of $>$ type such that their connection is $\check{\mathcal{C}}_{\text {fish }} \cdot\left(\underline{\boldsymbol{I}}_{\text {bub }}^{(1)}\right)^{2}$ have similar structure, although it is completely finite and do not affect UV divergence and renormalization.

Because of the similar color structure for terms in (7.44), it is possible to expand (7.44) on the color factors $\breve{\mathcal{C}}_{\text {fish }}$ written down in (7.46) with range $r=2,3$. Therefore, extracting kinematic coefficient of (7.44) gives

$$
\begin{array}{ll}
\text { Range-3: } & \mathcal{I}_{\mathrm{SU}(2), \mathrm{a}}^{(2)}(123)=-\mathcal{Z}^{(1)} \mathcal{I}_{\text {bub }}^{(1)}(12)+\underline{\mathcal{I}}_{\text {bub }}^{(1)}(12) \underline{\mathcal{I}}_{\text {bub }}^{(1)}(13)-\mathcal{Z}^{(2)}(123)+\mathcal{R}_{\mathrm{b}}^{(2)}(123), \\
\text { Range-2: } & \mathcal{I}_{\mathrm{SU}(2), \mathrm{b}}^{(2)}(12)=-\mathcal{Z}^{(1)} \mathcal{I}_{\text {bub }}^{(1)}(12)+\frac{1}{2}\left(\underline{\underline{I}}_{\text {bub }}^{(1)}(12)\right)^{2}-\mathcal{Z}^{(2)}(12)+\mathcal{R}_{\mathrm{b}}^{(2)}(12),
\end{array}
$$

where the renormalization constant and remainder should satisfy ${ }^{21}$

$$
\begin{array}{ll}
\mathcal{Z}^{(2)}(123)=\left.\boldsymbol{Z}_{123}^{(2)}\right|_{\check{\mathcal{C}}_{\text {fish }}^{r=3}}, & \mathcal{Z}^{(2)}(12)=\left.\boldsymbol{Z}_{12}^{(2)}\right|_{\check{\mathcal{C}}_{\text {fish }}^{r=2}}, \\
\mathcal{R}_{\mathrm{b}}^{(2)}(123)=\left.\boldsymbol{R}_{123, \mathrm{~b}}^{(2)}\right|_{\check{\mathcal{C}}_{\text {fish }}^{r=3}}, & \mathcal{R}_{\mathrm{b}}^{(2)}(12)=\left.\boldsymbol{R}_{12, \mathrm{~b}}^{(2)}\right|_{\check{\mathcal{C}}_{\text {fish }}^{r=2}} .
\end{array}
$$

Since all the elements in (7.50) are known, $\mathcal{Z}^{(2)}$ and $\mathcal{R}^{(2)}$ can be easily solved as

$$
\begin{aligned}
\mathcal{Z}^{(2)}(123)= & \frac{1}{2 \epsilon^{2}}-\frac{1}{2 \epsilon}, \\
\mathcal{Z}^{(2)}(12)= & \frac{1}{2 \epsilon^{2}}-\frac{1}{2 \epsilon}, \\
\mathcal{R}_{\mathrm{b}}^{(2)}(12)= & \frac{7}{2}-\log \left(-s_{12}\right), \\
\mathcal{R}_{\mathrm{b}}^{(2)}(123)= & \frac{7}{2}-\log \left(-s_{12}\right)+\frac{1}{2}\left(\log \left(-s_{123}\right)-2\right) \log \left(\frac{u}{v}\right) \\
& -\left(\operatorname{Li}_{2}(1-u)+\frac{1}{2} \log (u) \log (v)\right) .
\end{aligned}
$$

The two-loop dilatation operator can be obtained using $\mathbb{D}^{(2)}=4 \epsilon\left[\boldsymbol{Z}^{(2)}-\frac{1}{2}\left(\boldsymbol{Z}^{(1)}\right)^{2}\right]$. To do this, we first write down the complete form of renormalized constant

$$
\begin{aligned}
& \boldsymbol{Z}_{123}^{(2)}=\check{\mathcal{C}}_{\text {fish }}^{r=3}\left(\frac{1}{2 \epsilon^{2}}-\frac{1}{2 \epsilon}\right)+\left(a_{2}, p_{2} \leftrightarrow a_{3}, p_{3}\right), \\
& \boldsymbol{Z}_{12}^{(2)}=2 \check{\mathcal{C}}_{\text {fish }}^{r=2}\left(\frac{1}{2 \epsilon^{2}}-\frac{1}{2 \epsilon}\right)
\end{aligned}
$$

and $\left(\boldsymbol{Z}^{(1)}\right)^{2}$ is given in (D.4). Consequently, the (density) of dilatation operator reads ${ }^{22}$

$$
\left(\mathbb{D}^{(2)}\right)_{123}=-2 \check{\mathcal{C}}_{\text {fish }}^{r=3}, \quad\left(\mathbb{D}^{(2)}\right)_{12}=-4 \check{\mathcal{C}}_{\text {fish }}^{r=2} .
$$

\footnotetext{
${ }^{21}$ Here $\mathcal{Z}^{(2)}$ has no kinematic dependence. The (123) and (12) in argument are just used to distinguish the range.

${ }^{22}$ Recall that this dilatation density is derived under the momenta labeling $p_{1}^{Y} p_{2}^{X} p_{3}^{X}$ acting on 123 sites with fields $Y X X$.
} 
Given this, the $\mathrm{SU}(2)$ dilatation operator should be $\mathrm{e}^{23}$

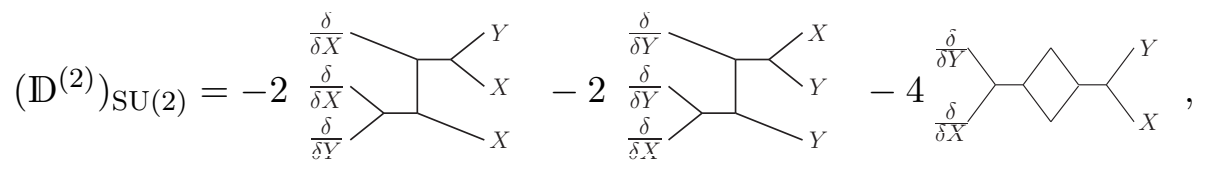

where the dilatation operator $\left(\mathbb{D}^{(2)}\right)_{\mathrm{SU}(2)}$ acts on operators like $\operatorname{tr}(X Y X Y \cdots)$ directly and bypass the process of momenta labeling of fields. This form is equivalent to the full-color dilatation operator in $\mathrm{SU}(2)$ Sector in [63].

Finally, let us briefly comment on remainders. $\boldsymbol{R}_{\mathrm{b}}^{(2)}$ have simple kinematic functions with transcendentality ranging form 2 to 0 as in (7.52). An interesting fact is that only $\boldsymbol{R}_{\mathrm{b}}^{(2)}$ have rational pieces (or say transcendentality-0 parts), and rational pieces satisfy

$$
\mathbb{D}^{(2)}=-\left.\frac{4}{7} \boldsymbol{R}^{(2)}\right|_{\operatorname{deg}-0}
$$

where $\left.\boldsymbol{R}\right|_{\text {deg-m }}$ denotes transcendentality- $m$ part of remainders.

Divergence cancellation in $\boldsymbol{I}_{\text {mix }}^{(2)}$. Finally we turn to the $\boldsymbol{I}_{\text {mix }}^{(2)}$ part of our analysis on $\mathrm{SU}(2)$ form factor. This part serves to provide a consistency check as well as to give the final third part of the remainder. Similar to our previous discussion for $\boldsymbol{I}_{\text {fish }}$, we can also expand the subtraction equation (7.45) for $\boldsymbol{I}_{\text {mix }}$ on the color factors $\check{\mathcal{C}}_{\text {mix }}$ in (7.47).

Hence, it is convenient to write down equations parallel to (7.50) as follows

$$
\begin{array}{ll}
\text { Range-3: } & \mathcal{I}_{\mathrm{SU}(2), \mathrm{c}}^{(2)}(123)=\mathcal{I}_{\text {tri }}^{(1)}(23) \mathcal{I}_{\text {bub }}^{(1)}(12)+V(\epsilon)+\mathcal{R}_{\mathrm{c}}(123), \\
\text { Range-2: } & \mathcal{I}_{\mathrm{SU}(2), \mathrm{d}}^{(2)}(12)=\mathcal{I}_{\text {tri }}^{(1)}(12) \mathcal{I}_{\text {bub }}^{(1)}(12)+\tilde{f}^{(2)}(\epsilon) \underline{\mathcal{I}}_{\text {bub }}^{(1)}(12)+2 V(\epsilon)+\mathcal{R}_{\mathrm{c}}(12),
\end{array}
$$

where we have introduced another auxiliary quantity $V \equiv \zeta_{2} / \epsilon$ similar to (7.26). And the final task of the cancellation of auxiliary $V$, beyond density level, is discussed in appendix F.2.

The results of remainders are

$$
\begin{aligned}
\mathcal{R}_{\mathrm{c}}^{(2)}(12)= & -4 \zeta_{2}+8 \zeta_{3}, \\
\left.\mathcal{R}_{\mathrm{c}}^{(2)}(123)\right|_{\text {deg-3 }}= & {\left[-\operatorname{Li}_{3}\left(-\frac{u}{w}\right)+\log (u) \operatorname{Li}_{2}\left(\frac{v}{1-u}\right)-\frac{1}{2} \log (u) \log (1-u) \log \left(\frac{w^{2}}{1-u}\right)\right.} \\
& \left.+\frac{1}{2} \operatorname{Li}_{2}\left(-\frac{u v}{w}\right)+\frac{1}{2} \log (u) \log (v) \log (w)+\frac{1}{12} \log ^{3}(w)+(u \leftrightarrow v)\right] \\
& +\operatorname{Li}_{3}(1-u)-\operatorname{Li}_{3}(v)+\frac{1}{2} \log ^{2}(v) \log \left(\frac{1-v}{u}\right)-\zeta_{2} \log \left(\frac{u^{2}}{w}\right)-3 \zeta_{3}, \\
\left.\mathcal{R}_{\mathrm{c}}^{(2)}(123)\right|_{\text {deg-2 }}= & -\left(\operatorname{Li}_{2}(1-u)+\frac{1}{2} \log ^{2}(u)\right)+2 \zeta_{2} .
\end{aligned}
$$

\footnotetext{
${ }^{23}$ Here the $\delta / \delta X$ is understood similar to the $\delta / \delta T^{a}$ in section. 3.1 and the analytic expression for, e.g. the first term in $(7.55)$ is $f^{Y X z_{1}} f^{z_{1} z_{2} \check{X}} f^{z_{3} z_{2} X} f^{\check{Y} X z_{3}}=\operatorname{tr}([[Y, X], \check{X}][[\check{Y}, \check{X}], X])$, similar to the notation used in [63].
} 
We observe that these remainders (of degree less than 4) are compatible with the planar remainders in $[36,38]$ up to some $\zeta_{2} \times \log$ and $\zeta_{3}$ terms. This can be explained by the observation that the full-color density in (7.47) behaves like the same blocks appearing in planar densities, for example in [36], by dressing color factors.

\subsection{Comment on new features of non-planar subtractions and remainders}

Through the discussion in this section, we have encountered several new features originating from non-planarity, including the divergent $\boldsymbol{H}^{(2)}$ terms and the finite non-planar remainder $\boldsymbol{R}^{\mathrm{NP}}$. Both of these quantities can be explained by the breaking of parity symmetry for the kinematic part of non-planar color factors, such as $\left[\mathcal{I}^{(2)}\right]_{\check{\mathcal{D}}_{19}}$ for BPS form factors. Comparing (5.41) and (5.40), it is clear that (5.40) has cyclic symmetry while (5.41) has a parity symmetry $\left(p_{1} \leftrightarrow p_{3}\right)$. Schematically, the part violating this parity symmetry in $(5.40)$ can be regarded as

$$
\mathcal{I}^{\text {anti-sym }} \equiv \sum_{\sigma \in S_{3}}(-1)^{\sigma} \Longrightarrow \sim_{\sigma\left(p_{3}\right)}^{+\infty \sigma\left(p_{2}\right)} .
$$

One can show that the divergent part of (7.59) is just $\mathcal{H}^{(2)}$, i.e. the kinematic part of $\boldsymbol{H}^{(2)}$, up to certain overall number. Such an argument gives us a heuristic reasoning about the origin of new non-planar contributions, and we will discuss them in detail below.

We start from $\boldsymbol{H}^{(2)}$ terms first. These $\boldsymbol{H}$ terms should have only $\epsilon^{-1}$ divergence and can be regarded as commutators of one-loop quantities. It is the non-commutative property of full-color one-loop quantities that results in such $\boldsymbol{H}$ terms.

A clear example is the $\boldsymbol{H}^{(2)}$ terms in BPS form factors, for example in (7.16), whose density, $\boldsymbol{H}_{123}^{(2)}$, can be written as a commutator between finite and divergent part of one-loop density

$$
\boldsymbol{H}_{123}^{(2)}=\frac{1}{2}\left(\left.\left.\boldsymbol{I}_{23}^{(1)}\right|_{\text {fin }} \boldsymbol{I}_{12}^{(1)}\right|_{\text {div }}-\left.\left.\boldsymbol{I}_{12}^{(1)}\right|_{\text {fin }} \boldsymbol{I}_{23}^{(1)}\right|_{\text {div }}\right)+\operatorname{cyc}\left(p_{1}, p_{2}, p_{3}\right),
$$

where $\boldsymbol{I}_{i j}$ is the one-loop BPS correction in appendix D, and subscript 'fin'('div') means taking the finite(divergent) part. The concrete computation have been given in (F.4) in appendix F.1 and we just highlight the triple-log structure here

$$
\left[\mathcal{H}^{(2)}\right]_{\check{\mathcal{D}}_{19}}(123)=\frac{1}{4 \epsilon} \log \left(\frac{-s_{12}}{-s_{23}}\right) \log \left(\frac{-s_{23}}{-s_{13}}\right) \log \left(\frac{-s_{13}}{-s_{12}}\right) .
$$

This commutator expression is compatible with the $\hat{\mathbf{H}}^{(2)}$ operator in [86], where $\hat{\mathbf{H}}^{(2)}$ is also interpreted as a commutator. The form of (7.60) also inspires us that it is possible to deform the one-loop square to a close function as:

$$
\frac{1}{2}\left(\underline{\boldsymbol{I}}^{(1)}\right)^{2}+\boldsymbol{H}^{(2)}=\frac{1}{2}\left(\left.\underline{\boldsymbol{I}}^{(1)}\right|_{\mathrm{fin}}\right)^{2}+\frac{1}{2}\left(\left.\underline{\boldsymbol{I}}^{(1)}\right|_{\mathrm{div}}\right)^{2}+\left(\left.\underline{\boldsymbol{I}}^{(1)}\right|_{\mathrm{fin}}\right)\left(\left.\underline{\boldsymbol{I}}^{(1)}\right|_{\mathrm{div}}\right)
$$

such that if we use the r.h.s. to replace $\frac{1}{2}\left(\underline{\boldsymbol{I}}^{(1)}\right)^{2}$ in the subtraction formula (7.8), then the $\boldsymbol{H}^{(2)}$ function is no-longer needed. 
As claimed in section 7.3 , the $\boldsymbol{H}$ functions for $\mathrm{SU}(2)$ form factors are similar but slightly different from BPS form factors. More concretely,

$$
\boldsymbol{H}_{\mathrm{SU}(2)}^{(2)}=\boldsymbol{H}_{\mathrm{BPS}}^{(2)}+\boldsymbol{H}_{\mathrm{mix}}^{(2)} .
$$

The only point deserving notice for $\boldsymbol{H}_{\mathrm{BPS}}^{(2)}$ is that only some configurations for a certain color factor have such triple-log divergence, similar to the discussion in section 5.3. Here $\boldsymbol{H}_{\text {mix }}$ has the following kinematic parts

$$
\begin{aligned}
& {\left[\left(\mathcal{H}_{\text {mix }}^{(2)}\right)_{Y X X}^{Y X X}\left(p_{1}^{Y}, p_{2}^{X}, p_{3}^{X}\right)\right]_{\check{\mathcal{D}}_{19}}=\frac{-2+\log \left(s_{23}\right)}{\epsilon} \log \left(\frac{-s_{12}}{-s_{13}}\right),} \\
& {\left[\left(\mathcal{H}_{\text {mix }}^{(2)}\right)_{Y X X}^{X Y X}\left(p_{1}^{X}, p_{2}^{Y}, p_{3}^{X}\right)\right]_{\check{\mathcal{D}}_{19}}=\frac{-2+\log \left(s_{12}\right)}{\epsilon} \log \left(\frac{-s_{13}}{-s_{23}}\right),} \\
& {\left[\left(\mathcal{H}_{\text {mix }}^{(2)}\right)_{Y X X}^{X X Y}\left(p_{1}^{X}, p_{2}^{X}, p_{3}^{Y}\right)\right]_{\check{\mathcal{D}}_{19}}=\frac{-2+\log \left(s_{13}\right)}{\epsilon} \log \left(\frac{-s_{23}}{-s_{12}}\right),}
\end{aligned}
$$

with a commutator intepretation $\frac{1}{2}\left[\left.\underline{\boldsymbol{I}}_{\text {tri }}^{(1)}\right|_{\text {div }}, \underline{\boldsymbol{I}}_{\text {bub }}^{(1)}\right] \cdot{ }^{24}$ As a result, the deformation in (7.62) also seems to be applicable for $\mathrm{SU}(2)$ form factors. It would be interesting to check if such a deformation also suits more general form factors in other sectors.

Let us further discuss the new contribution for non-planar remainders $\boldsymbol{R}^{\mathrm{NP}}$ and its component given in (7.37). As mentioned in section $7.2, \boldsymbol{R}^{\mathrm{NP}}$ have cyclic symmetry and anti-parity symmetry while planar densities have parity symmetry. The breaking of parity symmetry in the non-planar content explains the origination of $\boldsymbol{R}^{\mathrm{NP}}$. We also would like to stress that the discrepancy between planar and non-planar contributions is still consistent with the maximal transcendentality principle first introduced in study of anomalous dimensions in $[112,113]$ and later observed for the remainder functions of various form factors $[11,14,36-41,114-119]$. Therefore, we expect that the new non-planar contribution will also be a universal function that appears in generic (non-planar) gauge theories.

Finally, we discuss the cases when the non-planar correction has non-trivial contribute after summing over all densities in the full form factor. For $\boldsymbol{H}^{(2)}$ in the BPS case, the color structure of (7.16) is special, which is

$$
\check{\mathcal{C}}^{\text {anti-sym }} \equiv \sum_{\sigma \in S_{3}}(-1)^{\sigma} \check{\mathcal{D}}_{\Delta_{2}}(\sigma) .
$$

Action of $\check{\mathcal{C}}^{\text {anti-sym }}$ on tree-level form factors shows that only length $\geq 6$ operators, regardless of their trace structures, can possibly give non-vanishing contributions. Such a structure certainly relies on the property of identical fields for BPS form factors.

Similar observation holds for other quantities for SU(2) form factors, for example the $\boldsymbol{H}$ terms and remainder $\boldsymbol{R}$. Expanding on DDM basis defined in section 5.1, the kinematics coefficient of $\check{\mathcal{D}}_{19}$ has a non-vanishing BPS part only for the $Y X X \rightarrow Y X X$ configuration. Consequently, the color coefficient of triple-log divergence in (7.61) can appear in form factors of operators with length $\geq 4$, which is not as strict as BPS form factors starting from length-6. Likewise, four-point amplitudes in QCD also have $\boldsymbol{H}$ terms contribution, see $[86,90]$.

\footnotetext{
${ }^{24} \underline{\boldsymbol{I}}_{\mathrm{bub}}$ is finite, and its commutator with finite part of $\underline{\boldsymbol{I}}_{\mathrm{tri}}$ is just finite simple log functions so we omit that.
} 


\section{Summary and outlook}

Comparing with the planar case, the study of non-planar sector of gauge theories remains a much harder problem. One main complication is that the color factors have to be taken into account. The correlation between color and kinematic degrees of freedom typically makes the structures much more intricate. Besides, certain powerful symmetries in the planar limit, such as integrability in $\mathcal{N}=4 \mathrm{SYM}$, are no longer applicable. In this paper, we consider non-planar form factors of generic high-length operators in $\mathcal{N}=4 \mathrm{SYM}$, which are an interesting class of observables that capture the information of both off-shell anomalous dimensions and on-shell amplitudes. To probe the non-planar sector efficiently, we apply both the on-shell unitarity method and color-kinematics duality, which represents the ideas of dealing with the full-color problem in a decoupled or entangled manner, respectively. Below we recapitulate the main results of this paper.

(1) To simplify the problem, we make a careful analysis on color decomposition, using both trace and trivalent (DDM) basis, to disentangle color and spacetime degrees of freedom. Since we consider form factors of high-length operators, to simplify the structure, we also decompose the loop correction in terms of density functions. These decompositions help to reduce the full computation to a small set of kinematic basis functions.

(2) We develop unitarity method with non-planar cuts to compute kinematic functions of form factors. Explicit two-loop results for BPS and SU(2) form factors are obtained. For the SU(2) case, the supersymmetric Ward identities are used to simplify the calculation.

(3) Color-kinematics duality, as an alternative way to study non-planar correction, is also considered. In this strategy, the symmetry relations inherited from the color factors play a (bonus) role to simplify the computation of kinematic parts. We construct the explicit CK-dual representation for two-loop density corrections of half-BPS form factors. An interesting observation is that certain scaleless integrals are needed in order to satisfy the CK duality.

(4) After integration, an analysis on full-color IR structure is performed. In contrast to the planar BDS ansatz, the full-color IR divergence contains a new non-planar contribution at $1 / \epsilon$ order (the $\boldsymbol{H}^{(2)}$ terms), which provides an independent check of the known dipole formula for two-loop IR divergences.

(5) As for the finite remainder function, we obtain a new non-planar contribution in the maximally transcendental part. This new contribution can be expressed in terms of classical polylogarithms and has a similar color structure and non-planar origin as the $\boldsymbol{H}^{(2)}$ term in the divergence part.

(6) Finally, the full-color $\mathrm{SU}(2)$ dilatation operator is also derived and is consistent with the previously known result, which also serves as a check for our $\mathrm{SU}(2)$ calculation. 
We hope the above concrete studies and results will open an avenue to explore the non-planar sector in gauge theories, in particular for the off-shell operators using on-shell amplitudes-inspired techniques. Let us mention a few generalizations and other problems that can be considered based on the study of this paper. First, it would be interesting to generalize the present study to operators in more generic sectors and also to higher loops. For some previous non-planar studies of anomalous dimension and correlation functions, see e.g. [63, 63, 120-128]. Second, the non-planar data would be useful for the study of possible hidden symmetries in the non-planar sector. A promising direction appears to be the Hexagon program which suggests that the integrability may be still applicable beyond the planar sector for correlation functions [129-133]. (See other integrability related studies in e.g. $[134,135]$.) Another important structure to explore is the color-kinematics duality, in particular for form factors of general non-BPS operators at two loops. Furthermore, the form factors at strong coupling via AdS/CFT have been studied in [4, 5, 12], and more recently, the Pentagon OPE method has been generalized to form factor [136]. It would be certainly interesting to have a non-planar generalization (see a related study for amplitudes [137]). Finally, although we focus on $\mathcal{N}=4$ SYM in this paper, the on-shell method is certainly applicable to form factors of high-dimensional operators in more generic gauge theories such as QCD, see e.g. [138].

\section{Acknowledgments}

We would like to thank Siyuan Zhang for discussions. This work is supported in part by the National Natural Science Foundation of China (Grants No. 11822508, 11947302, 11935013), and by the Key Research Program of Frontier Sciences of CAS under Grant No. QYZDB-SSW-SYS014. We also thank the support of the HPC Cluster of ITP-CAS.

\section{A Two-loop DDM basis}

In this appendix, we provide the explicit DDM basis $\check{\mathcal{D}}_{i}$ with $i=1, \ldots, 27$ for two-loop form factors that are defined in (5.4)-(5.7) in section 5.1:

$$
\begin{aligned}
& \check{\mathcal{D}}_{1}=f^{\check{a}_{l_{1}} a_{1} \mathrm{x}_{1}} f^{\mathrm{x}_{1} a_{2} \mathrm{x}_{2}} f^{\mathrm{x}_{2} \mathrm{x}_{3} \check{a}_{l_{2}}} f^{\mathrm{x}_{3} a_{3} \check{a}_{l_{3}}}, \\
& \check{\mathcal{D}}_{2}=f^{\check{a}_{l_{2}} a_{1} \mathrm{x}_{1}} f^{\mathrm{x}_{1} a_{2} \mathrm{x}_{2}} f^{\mathrm{x}_{2} \mathrm{x}_{3} \check{a}_{l_{3}}} f^{\mathrm{x}_{3} a_{3} \check{a}_{l_{1}}}, \\
& \check{\mathcal{D}}_{3}=f^{\check{a}_{l_{3}} a_{1} \mathrm{x}_{1}} f^{\mathrm{x}_{1} a_{2} \mathrm{x}_{2}} f^{\mathrm{x}_{2} \mathrm{x}_{3} \check{a}_{l_{1}}} f^{\mathrm{x}_{3} a_{3} \check{a}_{l_{2}}}, \\
& \check{\mathcal{D}}_{4}=f^{\check{a}_{l_{1}} a_{1} \mathrm{x}_{1}} f^{\mathrm{x}_{1} a_{3} \mathrm{x}_{2}} f^{\mathrm{x}_{2} \mathrm{x}_{3} \check{a}_{l_{2}}} f^{\mathrm{x}_{3} a_{2} \check{a}_{l_{3}}}, \\
& \check{\mathcal{D}}_{5}=f^{\check{a}_{l_{2}} a_{1} \mathrm{x}_{1}} f^{\mathrm{x}_{1} a_{3} \mathrm{x}_{2}} f^{\mathrm{x}_{2} \mathrm{x}_{3} \check{a}_{l_{3}}} f^{\mathrm{x}_{3} a_{2} \check{a}_{l_{1}}} \text {, } \\
& \check{\mathcal{D}}_{6}=f^{\check{a}_{l_{3}} a_{1} \mathrm{x}_{1}} f^{\mathrm{x}_{1} a_{3} \mathrm{x}_{2}} f^{\mathrm{x}_{2} \mathrm{x}_{3} \check{a}_{l_{1}}} f^{\mathrm{x}_{3} a_{2} \check{a}_{l_{2}}}, \\
& \check{\mathcal{D}}_{7}=f^{\check{a}_{l_{1}} a_{2} \mathrm{x}_{1}} f^{\mathrm{x}_{1} a_{1} \mathrm{x}_{2}} f^{\mathrm{x}_{2} \check{a}_{l_{2}} \mathrm{x}_{3}} f^{\mathrm{x}_{3} a_{3} \check{a}_{l_{3}}}, \\
& \check{\mathcal{D}}_{8}=f^{\check{a}_{l_{2}} a_{2} \mathrm{x}_{1}} f^{\mathrm{x}_{1} a_{1} \mathrm{x}_{2}} f^{\mathrm{x}_{2} \mathrm{x}_{3} \check{a}_{l_{3}}} f^{\mathrm{x}_{3} a_{3} \check{a}_{l_{1}}}, \\
& \check{\mathcal{D}}_{9}=f^{\check{a}_{l_{3}} a_{2} \mathrm{x}_{1}} f^{\mathrm{x}_{1} a_{1} \mathrm{x}_{2}} f^{\mathrm{x}_{2} \mathrm{x}_{3} \check{a}_{l_{1}}} f^{\mathrm{x}_{3} a_{3} \check{a}_{l_{2}}}, \\
& \check{\mathcal{D}}_{10}=f^{\check{a}_{l_{1}} a_{2} \mathrm{x}_{1}} f^{\mathrm{x}_{1} a_{3} \mathrm{x}_{2}} f^{\mathrm{x}_{2} \mathrm{x}_{3} \check{a}_{l_{2}}} f^{\mathrm{x}_{3} a_{1} \check{a}_{l_{3}}}, \\
& \check{\mathcal{D}}_{11}=f^{\check{a}_{l_{2}} a_{2} \mathrm{x}_{1}} f^{\mathrm{x}_{1} a_{3} \mathrm{x}_{2}} f^{\mathrm{x}_{2} \mathrm{x}_{3} \check{a}_{l_{3}}} f^{\mathrm{x}_{3} a_{1} \check{a}_{l_{1}}}, \\
& \check{\mathcal{D}}_{12}=f^{\check{a}_{l_{3}} a_{2} \mathrm{x}_{1}} f^{\mathrm{x}_{1} a_{3} \mathrm{x}_{2}} f^{\mathrm{x}_{3} \mathrm{x}_{2} \check{a}_{l_{1}}} f^{\mathrm{x}_{3} a_{1} \check{a}_{l_{2}}}, \\
& \check{\mathcal{D}}_{13}=f^{\check{a}_{l_{1}} a_{3} \mathrm{x}_{1}} f^{\mathrm{x}_{1} a_{1} \mathrm{x}_{2}} f^{\mathrm{x}_{2} \mathrm{x}_{3} \check{a}_{l_{2}}} f^{\mathrm{x}_{3} a_{2} \check{a}_{l_{3}}}, \\
& \check{\mathcal{D}}_{14}=f^{\check{a}_{l_{2}} a_{3} \mathrm{x}_{1}} f^{\mathrm{x}_{1} a_{1} \mathrm{x}_{2}} f^{\mathrm{x}_{2} \mathrm{x}_{3} \check{a}_{l_{3}}} f^{\mathrm{x}_{3} a_{2} \check{a}_{l_{1}}}, \\
& \check{\mathcal{D}}_{15}=f^{\check{a}_{l_{3}} a_{3} \mathrm{x}_{1}} f^{\mathrm{x}_{1} a_{1} \mathrm{x}_{2}} f^{\mathrm{x}_{2} \mathrm{x}_{3} \check{a}_{l_{1}}} f^{\mathrm{x}_{3} a_{2} \check{a}_{l_{2}}}, \\
& \check{\mathcal{D}}_{16}=f^{\check{a}_{l_{1}} a_{3} \mathrm{x}_{1}} f^{\mathrm{x}_{1} a_{2} \mathrm{x}_{2}} f^{\mathrm{x}_{2} \mathrm{x}_{3} \check{a}_{l_{2}}} f^{\mathrm{x}_{3} a_{1} \check{a}_{l_{3}}}, \\
& \check{\mathcal{D}}_{17}=f^{\check{a}_{l_{2}} a_{3} \mathrm{x}_{1}} f^{\mathrm{x}_{1} a_{2} \mathrm{x}_{2}} f^{\mathrm{x}_{2} \mathrm{x}_{3} \check{a}_{l_{3}}} f^{\mathrm{x}_{3} a_{1} \check{a}_{l_{1}}}, \\
& \check{\mathcal{D}}_{18}=f^{\check{a}_{l_{3}} a_{3} \mathrm{x}_{1}} f^{\mathrm{x}_{1} a_{2} \mathrm{x}_{2}} f^{\mathrm{x}_{2} \mathrm{x}_{3} \check{a}_{l_{1}}} f^{\mathrm{x}_{3} a_{1} \check{a}_{l_{2}}}, \\
& \check{\mathcal{D}}_{19}=f^{a_{1} \check{a}_{l_{1} \mathrm{x}_{1}}} f^{a_{2} \check{a}_{l_{2} \mathrm{x}_{2}}} f^{a_{3} \check{a}_{l_{3} \mathrm{x}_{3}}} f^{\mathrm{x}_{1} \mathrm{x}_{2} \mathrm{x}_{3}}, \\
& \check{\mathcal{D}}_{20}=f^{a_{1} \check{a}_{l_{1}} \mathrm{x}_{1}} f^{a_{3} \check{a}_{l_{2}} \mathrm{x}_{2}} f^{a_{2} \check{a}_{l_{3}} \mathrm{x}_{3}} f^{\mathrm{x}_{1} \mathrm{x}_{2} \mathrm{x}_{3}}, \\
& \check{\mathcal{D}}_{21}=f^{a_{2} \check{a}_{l_{1}} \mathrm{x}_{1}} f^{a_{1} \check{a}_{l_{2}} \mathrm{x}_{2}} f^{a_{3} \check{a}_{l_{3}} \mathrm{x}_{3}} f^{\mathrm{x}_{1} \mathrm{x}_{2} \mathrm{x}_{3}}, \\
& \check{\mathcal{D}}_{22}=f^{a_{2} \check{a}_{l_{1}} \mathrm{x}_{1}} f^{a_{3} \check{a}_{l_{2}} \mathrm{x}_{2}} f^{a_{1} \check{a}_{l_{3} \mathrm{x}_{3}}} f^{\mathrm{x}_{1} \mathrm{x}_{2} \mathrm{x}_{3}},
\end{aligned}
$$




$$
\begin{aligned}
& \check{\mathcal{D}}_{23}=f^{a_{3} \check{a}_{l_{1}} \mathrm{x}_{1}} f^{a_{1} \check{a}_{l_{2}} \mathrm{x}_{2}} f^{a_{2} \check{a}_{l_{3}} \mathrm{x}_{3}} f^{\mathrm{x}_{1} \mathrm{x}_{2} \mathrm{x}_{3}}, \quad \check{\mathcal{D}}_{24}=f^{a_{3} \check{a}_{l_{1}} \mathrm{x}_{1}} f^{a_{2} \check{a}_{l_{2}} \mathrm{x}_{2}} f^{a_{1} \check{a}_{l_{3} \mathrm{x}_{3}}} f^{\mathrm{x}_{1} \mathrm{x}_{2} \mathrm{x}_{3}}
\end{aligned}
$$

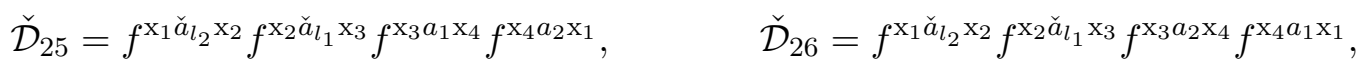

$$
\begin{aligned}
& \check{\mathcal{D}}_{27}=f^{\mathrm{x}_{1} \check{a}_{l_{2}} \mathrm{x}_{2}} f^{\mathrm{x}_{2} a_{2} \mathrm{x}_{3}} f^{\mathrm{x}_{3} a_{1} \mathrm{x}_{4}} f^{\mathrm{x}_{4} \check{a}_{l_{1}} \mathrm{x}_{1}} .
\end{aligned}
$$

Below we briefly comment on how to relate the BPS form factor from CK-dual form in section 6 to the result in section 5.2, since they have apparently different color structures. Consider the CK-dual represeduntation (6.25), the permutations of internal lines $\sigma^{i}$ action on $\check{\mathcal{C}}_{1}$ belong to $S_{3}$, while in the DDM basis (5.4) one has only $\sigma^{i} \in \mathbb{Z}_{3}$. The new permutations in CK form, namely $\sigma_{i} \in S_{3} \backslash \mathbb{Z}_{3}$, can be expressed in terms of DDM basis (5.4). For example, using the color Jacobi relation, one has

$$
\check{\mathcal{D}}_{\Delta_{1}(\mathbf{1}, \mathbf{1})}-\left.\check{\mathcal{D}}_{\Delta_{1}(\mathbf{1}, \mathbf{1})}\right|_{\text {refl. }}=\check{\mathcal{D}}_{\Delta_{2}(\mathbf{1})},
$$

where "refl." represents the reflection that maps $\left\{\left\{l_{1}, l_{2}, l_{3}\right\},\left\{p_{1}, p_{2}, p_{3}\right\}\right\}$ to $\left\{\left\{l_{3}, l_{2}, l_{1}\right\},\left\{p_{3}, p_{2}, p_{1}\right\}\right\}$, with which one can get the permutations $\sigma_{i} \in S_{3} \backslash \mathbb{Z}_{3}$ from $\mathbb{Z}_{3}$. Eq. (A.2) can be also given in a diagrammatic form

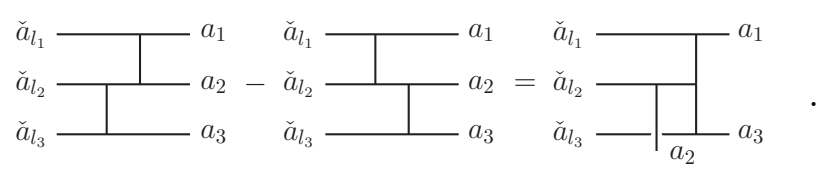

With such equations, one can expand the CK-dual form (6.25) on the DDM basis defined in section 5.1 and then compare it with the result in section 5.2 .

Two-loop DDM basis of next-to-minimal form factors. As a generalization to the discussion in section 5.1, we consider further the two-loop next-to-minimal trivalent basis. For the range-3 density, we have the following two sets of trivalent color structures:

$$
\begin{aligned}
& \operatorname{Set}_{1}=\left\{\check{\mathcal{D}}_{\Delta_{1}}\left(\sigma^{e}, \sigma^{i}\right)\right\}_{\sigma^{i} \in S_{3}, \sigma^{e} \in S_{4} / \mathbb{Z}_{2}}=\left\{\sigma^{i} \sigma^{e} \cdot f^{\check{a}_{l_{1}} a_{1} \mathrm{x}_{1}} f^{\mathrm{x}_{1} a_{2} \mathrm{x}_{2}} f^{\mathrm{x}_{2} a_{3} \mathrm{x}_{3}} f^{\mathrm{x}_{3} \check{a}_{l_{2}} \mathrm{x}_{4}} f^{\left.\mathrm{x}_{4} a_{4} \check{a}_{l_{3}}\right\},}\right. \\
& \operatorname{Set}_{2}=\left\{\check{\mathcal{D}}_{\Delta_{2}}\left(\sigma^{e}, \sigma^{i}\right)\right\}_{\sigma^{i} \in S_{3}, \sigma^{e} \in \mathbb{Z}_{2} \times \mathbb{Z}_{4}}=\left\{\sigma^{i} \sigma^{e} \cdot f^{\check{a}_{l_{1}} a_{1} \mathrm{x}_{1}} f^{\check{a}_{l_{2}} a_{2} \mathrm{x}_{2}} f^{\check{a}_{l_{3}} a_{3} \mathrm{x}_{4}} f^{\mathrm{x}_{4} a_{4} \mathrm{x}_{3}} f^{\mathrm{x}_{1} \mathrm{x}_{2} \mathrm{x}_{3}}\right\},
\end{aligned}
$$

where $S_{4} / \mathbb{Z}_{2}$ means permutation of $\left\{a_{1}, a_{2}, a_{3}, a_{4}\right\} \bmod$ reflection, i.e. $\{1,2,3,4\} \leftrightarrow$ $\{4,3,2,1\}, \mathbb{Z}_{4} \times \mathbb{Z}_{2}$ represents cyclic permutaions of $\left\{a_{1}, a_{2}, a_{3}, a_{4}\right\}$ times exchanging the last two elements in the set, namely, exchanging the middle two lines in figure 14(b). Both of these choices are based on the symmetry of topology of diagrams. In this color basis, similar to the planar case, all color-stripped form factors in Set $_{1}$ can be one-to-one mapped to certain planar matrix elements. As a check, the number of above range-3 basis is $3 ! \times 4 ! / 2+3 ! \times 8=5$ ! , which is the same as the dimension of DDM basis for seven-point amplitude $\boldsymbol{A}_{7}^{(0)}$.

Similarly for the range- 2 color basis, one has

$$
\begin{aligned}
& \operatorname{Set}_{3}=\left\{\check{\mathcal{D}}_{\Delta_{3}}\left(\sigma_{e}\right)\right\}_{\sigma_{e} \in S_{3}}=\left\{\sigma_{e} \cdot f^{\mathrm{x}_{1} \check{a}_{l_{1}} \mathrm{x}_{2}} f^{\mathrm{x}_{2} \check{a}_{l_{2}} \mathrm{x}_{3}} f^{\mathrm{x}_{3} a_{1} \mathrm{x}_{4}} f^{\mathrm{x}_{4} a_{2} \mathrm{x}_{5}} f^{\mathrm{x}_{5} a_{3} \mathrm{x}_{1}}\right\}, \\
& \operatorname{Set}_{4}=\left\{\check{\mathcal{D}}_{\Delta_{4}}\left(\sigma_{e}\right)\right\}_{\sigma_{e} \in S_{3}}=\left\{\sigma_{e} \cdot f^{\mathrm{x}_{1} \check{a}_{l_{1} \mathrm{x}_{2}}} f^{\mathrm{x}_{2} a_{3} \mathrm{x}_{3}} f^{\mathrm{x}_{3} \check{a}_{l_{2}} \mathrm{x}_{4}} f^{\mathrm{x}_{4} a_{1} \mathrm{x}_{5}} f^{\mathrm{x}_{5} a_{2} \mathrm{x}_{1}}\right\} .
\end{aligned}
$$




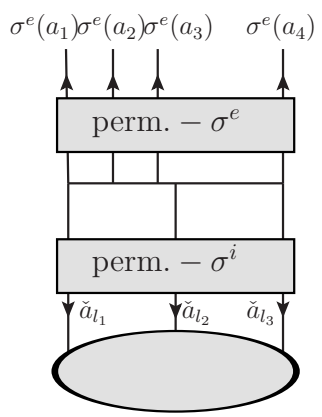

(a) Cubic Graph: $\check{\mathcal{D}}_{\Delta_{1}}$

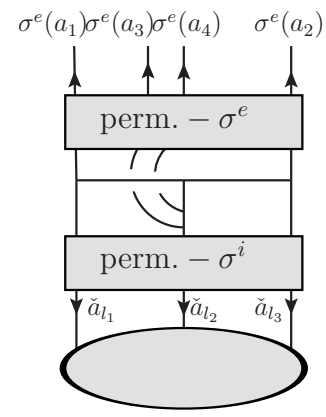

(b) Cubic Graph: $\check{\mathcal{D}}_{\Delta_{2}}$

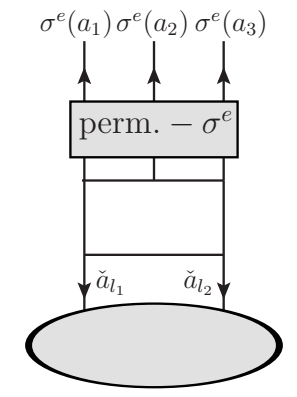

(c) Cubic Graph: $\check{\mathcal{D}}_{\Delta_{3}}$

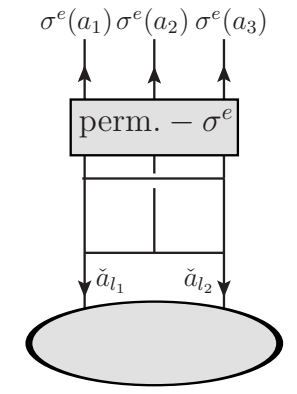

(d) Cubic Graph: $\check{\mathcal{D}}_{\Delta_{4}}$

Figure 14. Cubic Graphs for next-to-minimal two-loop correction densities.

The diagrammatic representation of (A.4)-(A.5) are given in figure 14. We can also check the counting of range- 2 basis as $2 \times 3 !=4 ! / 2$, which is equal to the number of elements in trivalent basis of one-loop five-point amplitudes.

\section{B Non-planar features of unitarity}

In this appendix, we consider some further details on non-planar unitarity following the discussion in section 4 . We will first address the question about how to classify the cut channels contributing to certain color factor $\mathcal{T}_{k}$, and then we discuss some subtle points about adding cut contribution from different cut channels.

Channels from different ordering. To classify cut channels corresponding to certain color factors, one can in principle consider all possible orderings of each tree blocks and sew them together. Below we will employ a procedure separately considering internal cut legs and external uncut legs .

In the first step, we consider the sewing of internal cut legs (and ignore the external legs). The crossing of internal lines can lead to $1 / N_{c}^{2}$ suppression. For the convenience of discussion, we introduce the crossing number, which is the minimal number of lines that have to be adjusted to reach a planar topology. For example, figure 15(a) is a planar example which has crossing number 0; figure 15(b) shows a connection with crossing number 1 , which means one can move one side of the red line to get a planar topology (although seemingly the red line cross two other cut lines); figure 15(c) shows a diagram with crossing number 2 , since it is necessary to adjust two red lines.

The crossing number defined above has a direct meaning in terms of color factors. Roughly speaking, the crossing number determines the number of traces in color factors. Here one can regard $N_{\mathrm{c}}=\operatorname{tr}(\mathbb{1})$ as a trace. Then the crossing number determines the number of trace plus the power of $N_{\mathrm{c}}$ (after simplifying the color factor). For connections with the same crossing number, the power of $N_{c}$ plus the number of traces at leading $N_{c}$ order are the same. Such a number is reduced by $2 j$ when increasing the crossing number by $j$. 


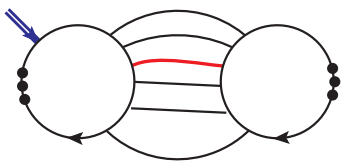

(a) Crossing number $=0$

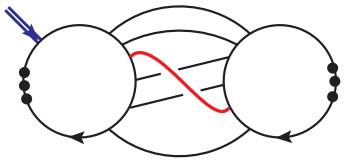

(b) Crossing number $=1$

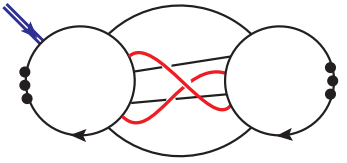

(c) Crossing number $=2$

Figure 15. Examples of connections with different crossing numbers. The circle with arrow represents a trace, and the black dots represent possible insertion of external legs.
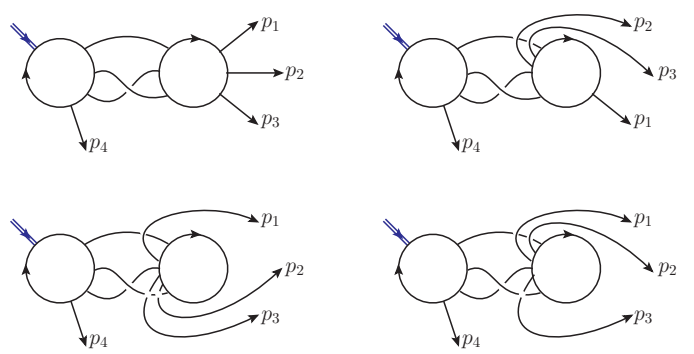
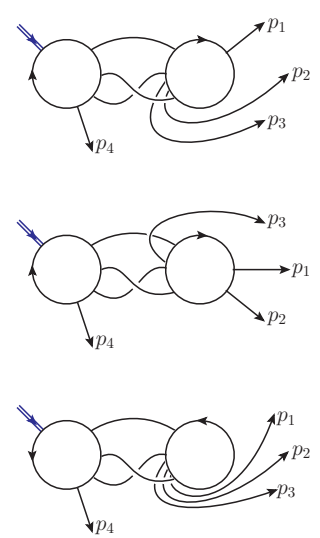
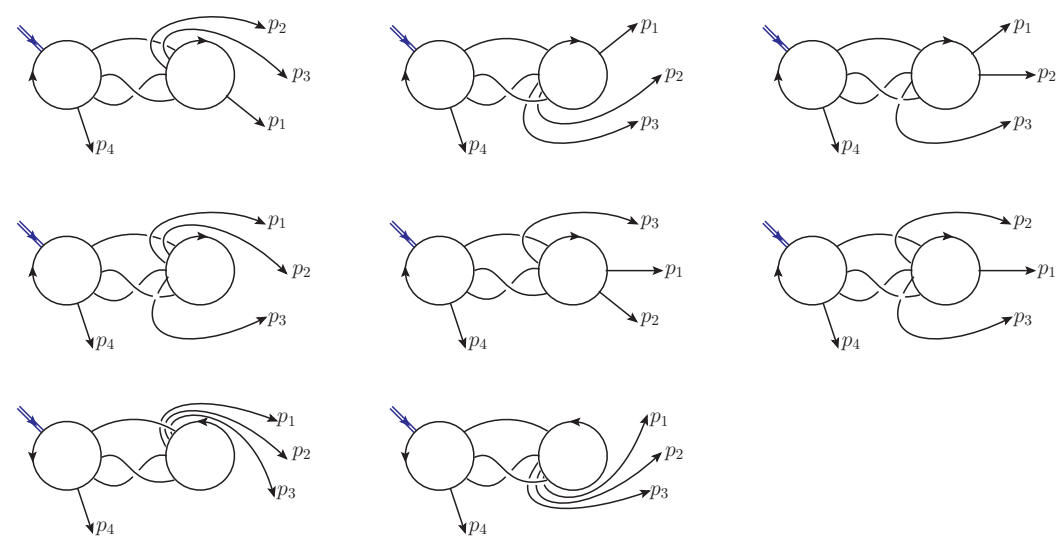

Figure 16. Triple-cut for two-loop minimal form factor $\mathcal{O}=\operatorname{tr}(X X X X)$ with crossing number 1 as in figure 15. These 10 figures correspond to different distributions of external legs $\left\{p_{1}, p_{2}, p_{3}\right\}$ (i.e. $\left.\mathrm{oP}\left\{p_{1}, p_{2}, p_{3}\right\}\right)$.

After fixing the sewing of internal legs, we next consider the distribution of external legs. For the case with crossing number 0, there is always a one-to-one correspondence between distributions of external legs and color factors $\mathcal{T}_{k}$. For higher crossing numbers, in contrast, there are possibilities that different ways of distributing external legs can contribute to the same color factor.

Consider the following example: a $s_{123}$-triple-cut of two-loop minimal form factor of a length-four operator, say $\mathcal{O}=\operatorname{tr}(X X X X)$, and our target color factor is $\mathcal{T}_{c}=\operatorname{tr}\left(a_{1} a_{2} a_{3} a_{4}\right)$. By carefully inspecting all possibilities, there are 10 configurations contribute to the same color factor $\mathcal{T}_{c}$, as listed in figure 16. To label the partitions of external legs within each block, we introduce $\mathrm{oP}_{k} S$ as ordered partitions of set $S$ into $k$ subsets. In the above example, we need $\{D, F, E\} \in \mathrm{oP}_{3}\left\{p_{1}, p_{2}, p_{3}\right\}$ explicitly given as

$$
\begin{aligned}
& \left\{\left\{p_{1}, p_{2}, p_{3}\right\}, \emptyset, \emptyset\right\},\left\{\emptyset,\left\{p_{1}, p_{2}, p_{3}\right\}, \emptyset\right\},\left\{\emptyset, \emptyset,\left\{p_{1}, p_{2}, p_{3}\right\}\right\}, \\
& \left\{\left\{p_{1}, p_{2}\right\},\left\{p_{3}\right\}, \emptyset\right\},\left\{\left\{p_{1}, p_{2}\right\}, \emptyset,\left\{p_{3}\right\}\right\},\left\{\emptyset,\left\{p_{1}, p_{2}\right\},\left\{p_{3}\right\}\right\}, \\
& \left.\left\{\left\{p_{1}\right\},\left\{p_{2}, p_{3}\right\}, \emptyset\right\},\left\{\left\{p_{1}\right\}, \emptyset,\left\{p_{2}, p_{3}\right\}\right\},\left\{\emptyset,\left\{p_{1}\right\},\left\{p_{2}, p_{3}\right\}\right\},\left\{\left\{p_{1}\right\},\left\{p_{2}\right\},\left\{p_{3}\right\}\right\}\right\} .
\end{aligned}
$$

Since the above partitions contribute to the same color factor, one can add them together in the cut integrand, in which one can consider applying amplitude identities (such as KK relations) to simplify the computation. 


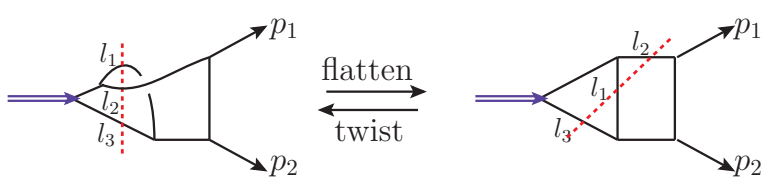

Figure 17. An example of twisted diagrams.

Comments on non-planar cut integrands. In the remaining part of this appendix, we consider a few features that are related to the non-planar cut integrands.

When constructing the non-planar cut integrand via the unitarity method, a complication is to identify integral topologies from the cut integrand. When the orderings of cut legs do not match with each other in different blocks, one typically encounters certain twisted topologies. It is then important to keep track the twisting ordering for integrands.

For example, consider the form factor of $\mathcal{O}=\operatorname{tr}(X X \cdots X)$ in the following cut channel:

$$
\begin{gathered}
\int \mathrm{dPS}_{3, l} \hat{\mathcal{F}}_{L+1}\left(-l_{1},-l_{2}, A,-l_{3}, B\right) \hat{\mathcal{A}}_{5}\left(l_{3}, l_{1}, l_{2}, p_{1}, p_{2}\right) \\
=\int \mathrm{dPS}_{3, l}\left(\cdots+\frac{s_{12}^{2}}{s_{1 l_{2}} s_{l_{1} l_{2}} s_{l_{1} l_{3}}}+\cdots\right) .
\end{gathered}
$$

The term written down explicitly on the r.h.s. in (B.2) corresponds to the cut integral representation as shown in figure 17.

In the left diagram in figure 17 , we preserve the twisting of cut legs so that the ordering of legs match that in planar tree blocks, for example, the ordering in $\mathcal{A}_{5}$ is $\left\{l_{3}, l_{1}, l_{2}, p_{1}, p_{2}\right\}$. This integral, after being flattened, is equivalent to a planar ladder integral in the r.h.s. of figure 17. We would like to stress that in the practical computation, it is important to keep track of twist ordering (as in the l.h.s. of figure 17) when we consider summarizing contribution from different cut channels. To illustrate this point, as a non-trivial example, we discuss in more detail the cuts of figure 8(b) and figure 8(c) below.

The cut channel of figure 8 (b) has been discussed in section 5.2, and the cut contribution has been given explicitly in (5.27). Parallel to the discussion of figure $8(\mathrm{~b})$, for the cut shown in figure $8(\mathrm{c})$, one has

$$
\begin{aligned}
{\left.\left[\mathcal{F}^{(2)}\right]_{\mathcal{T}_{t_{3}}}\right|_{s_{12}-\mathrm{DC}}=} & \int \mathrm{d} \hat{\mathrm{P} S} S_{3, l} \hat{\mathcal{F}}_{L+1, \mathrm{dens}}^{(0)}\left(p_{5},-l_{2},-l_{3}, p_{6}\right) \hat{\mathcal{A}}_{5}^{(0)}\left(p_{1}, l_{1}, p_{2}, l_{3}, l_{2}\right) \\
& +\left(\text { kinematic dependence on } p_{3} \text { and } p_{4}\right) .
\end{aligned}
$$

After subtracting contribution from range-3 density, the detected cut contribution to the density of $\check{\mathcal{D}}_{27}$ is similar to $(5.27)$ as

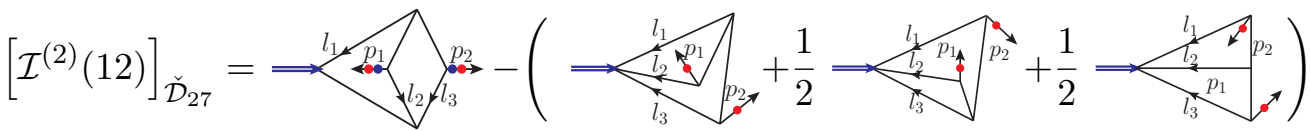

$$
\begin{aligned}
& + \text { (terms detected in other channels). }
\end{aligned}
$$

Let us compare (5.27) and (B.4): 
- The first term in (5.27) and (B.4) respectively correspond to the same integral with identical twisting. This twisted integral can be cut in the following two ways:

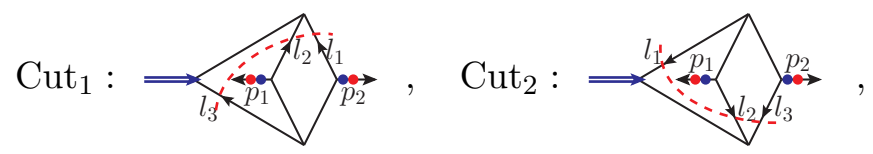

contributing to cut-channels of (5.27) and (B.4), respectively. In this case, the two cut channels detect the same integral.

As an illuminating analogy, one can recall the planar calculation, for example in (4.16): the "planar-ladder" integral has two cuts in the $s_{12}$ triple-cut channel:

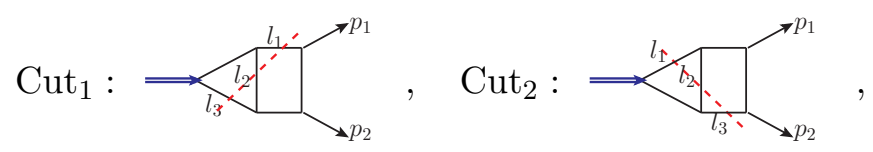

but both two cuts emerge in the same cut channel (with the same ordering).

- In contrast to the first term, the other terms in the parenthesis in (5.27) and (B.4) need to be treated differently. After being flattened, they are all equivalent to the same integral

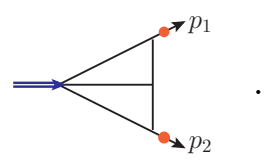

However, as shown in (5.27) and (B.4), they have different twisting structures. Therefore, they should be regarded as different integral contributions. In other words, one should sum up their coefficients to construct the full form factor integrand, and this explains the total coefficient $(-4)$ of this integral in (5.28).

Similar argument also holds for other cuts that we have used before. The point is that we must treat integrals with different twisting as different contributions and add them up. Another instance for illustrating this point is the factor 3 in (5.32).

\section{Six-point amplitudes}

In this appendix, we provide the explicit expressions of six-scalar tree amplitudes which are needed in the unitarity construction in section 4 and 5 . Some of them have been given in [36]. Here we give new elements that are required for full non-planar construction.

$$
\begin{aligned}
& \mathcal{A}_{6}^{(0)}\left(p_{1}^{X}, p_{2}^{X}, p_{3}^{X}, q_{3}^{\bar{X}}, q_{2}^{\bar{X}}, q_{1}^{\bar{X}}\right) \\
& \quad=-\frac{s_{p_{1} p_{2} p_{3}}}{s_{p_{1} q_{1}} s_{p_{3} q_{3}}}+\frac{s_{p_{2} p_{3}} s_{q_{1} q_{2}}}{s_{p_{1} q_{1}} s_{p_{3} q_{3}} s_{p_{2} p_{3} q_{3}}}+\frac{s_{p_{1} p_{2}} s_{q_{2} q_{3}}}{s_{p_{1} q_{1}} s_{p_{3} q_{3}} s_{p_{3} q_{2} q_{3}}}
\end{aligned}
$$




$$
\begin{aligned}
& \mathcal{A}_{6}^{(0)}\left(q_{1}^{\bar{X}}, p_{1}^{X}, p_{2}^{X}, q_{2}^{\bar{X}}, p_{3}^{X}, q_{3}^{\bar{X}}\right) \\
& =-\frac{s_{p_{1} p_{2}}}{s_{p_{2} q_{2}} s_{p_{3} q_{3}}}-\frac{s_{p_{1} p_{3}}}{s_{p_{1} q_{1}} s_{p_{2} q_{2}}}-\frac{s_{p_{2} p_{3}}}{s_{p_{1} q_{1}} s_{p_{3} q_{3}}}+\frac{s_{p_{1} p_{2} p_{3}}}{s_{p_{1} q_{1}} s_{p_{2} q_{2}}}+\frac{s_{p_{1} p_{2} p_{3}}}{s_{p_{1} q_{1}} s_{p_{3} q_{2}}}+\frac{s_{p_{1} p_{2} p_{3}}}{s_{p_{1} q_{1}} s_{p_{3} q_{3}}} \\
& +\frac{s_{q_{1} q_{3}} s_{p_{1} p_{2}}}{s_{p_{2} q_{2}} s_{p_{1} p_{2} q_{2}} s_{p_{3} q_{3}}}-\frac{s_{q_{2} q_{3}} s_{p_{1} p_{2}}}{s_{p_{1} q_{1}} s_{p_{1} p_{2} q_{1}} s_{p_{3} q_{2}}}-\frac{s_{q_{2} q_{3}} s_{p_{1} p_{2}}}{s_{p_{1} q_{1}} s_{p_{1} p_{2} q_{1}} s_{p_{3} q_{3}}}-\frac{s_{p_{2} p_{3}} s_{q_{1} q_{3}}}{s_{p_{1} q_{1}} s_{p_{3} q_{2}} s_{p_{2} p_{3} q_{2}}} \\
& -\frac{s_{p_{2} p_{3}} s_{q_{1} q_{3}}}{s_{p_{1} q_{1}} s_{p_{2} q_{2}} s_{p_{2} p_{3} q_{2}}}+\frac{s_{p_{1} p_{3}} s_{p_{1} p_{2}}}{s_{p_{1} q_{1}} s_{p_{2} q_{2}} s_{p_{3} q_{3}}}+\frac{s_{p_{2} p_{3}} s_{p_{1} p_{2}}}{s_{p_{1} q_{1}} s_{p_{2} q_{2}} s_{p_{3} q_{3}}}+\frac{s_{p_{3} q_{2}} s_{p_{1} p_{2}}}{s_{p_{1} q_{1}} s_{p_{2} q_{2}} s_{p_{3} q_{3}}} \\
& +\frac{s_{p_{2} p_{3}} s_{p_{1} q_{3}}}{s_{p_{1} q_{1}} s_{p_{2} q_{2}} s_{p_{3} q_{3}}}+\frac{s_{p_{1} p_{3}} s_{p_{2} q_{1}}}{s_{p_{1} q_{1}} s_{p_{2} q_{2}} s_{p_{3} q_{3}}}+\frac{s_{p_{1} p_{3}} s_{p_{2} p_{3}}}{s_{p_{1} q_{1}} s_{p_{2} q_{2}} s_{p_{3} q_{3}}} \\
& \mathcal{A}_{6}^{(0)}\left(p_{1}^{Y}, p_{2}^{X}, p_{3}^{X}, q_{3}^{\bar{X}}, q_{2}^{\bar{X}}, q_{1}^{\bar{Y}}\right) \\
& =-\frac{s_{p_{2} p_{3}} s_{q_{1} q_{2}}}{s_{p_{1} q_{1}} s_{p_{3} q_{3}} s_{p_{2} p_{3} q_{3}}}-\frac{s_{p_{1} p_{2}} s_{q_{2} q_{3}}}{s_{p_{1} q_{1}} s_{p_{3} q_{3}} s_{p_{3} q_{2} q_{3}}}+\frac{s_{p_{1} p_{2} p_{3}}}{s_{p_{1} q_{1}} s_{p_{3} q_{3}}} \\
& -\frac{s_{p_{2} p_{3}}}{s_{p_{3} q_{3}} s_{p_{2} p_{3} q_{3}}}-\frac{s_{q_{2} q_{3}}}{s_{q_{3} p_{3}} s_{p_{3} q_{2} q_{3}}}+\frac{1}{s_{p_{3} q_{3}}}, \\
& \mathcal{A}_{6}^{(0)}\left(p_{1}^{Y}, p_{2}^{X}, p_{3}^{X}, q_{1}^{\bar{Y}}, q_{2}^{\bar{X}}, q_{3}^{\bar{X}}\right) \\
& =-\frac{1}{s_{p_{1} p_{2} q_{3}}}, \\
& \mathcal{A}_{6}^{(0)}\left(p_{1}^{Y}, p_{2}^{X}, p_{3}^{X}, q_{3}^{\bar{X}}, q_{1}^{\bar{Y}}, q_{2}^{\bar{X}}\right) \\
& =\frac{s_{p_{2} p_{3}}}{s_{p_{3} q_{3}} s_{p_{2} p_{3} q_{3}}}+\frac{s_{q_{1} q_{3}}}{s_{p_{3} q_{3}} s_{p_{3} q_{3} q_{1}}}-\frac{1}{s_{p_{3} q_{3}}}+\frac{1}{s_{p_{3} q_{3} q_{1}}}, \\
& \mathcal{A}_{6}^{(0)}\left(q_{1}^{\bar{Y}}, p_{1}^{Y}, p_{2}^{X}, q_{2}^{\bar{X}}, q_{3}^{\bar{X}}, p_{3}^{X}\right) \\
& =-\frac{s_{p_{1} p_{2}}}{s_{p_{2} q_{2}} s_{p_{1} p_{2} q_{2}}}+\frac{s_{p_{2} p_{3}}}{s_{p_{2} q_{2}} s_{p_{2} p_{3} q_{2}}}-\frac{s_{p_{3} q_{2}}}{s_{p_{1} p_{2} q_{1}} s_{p_{3} q_{3}}}+\frac{s_{p_{3} q_{1}}}{s_{p_{1} p_{2} q_{2}} s_{p_{3} q_{3}}} \\
& +\frac{s_{p_{2} p_{3}}}{s_{p_{3} q_{2}} s_{p_{2} p_{3} q_{2}}}-\frac{s_{p_{3} q_{3}}}{s_{p_{3} q_{2}} s_{p_{3} q_{2} q_{3}}}-\frac{2}{s_{p_{3} q_{2} q_{3}}}-\mathcal{A}_{6}^{(0)}\left(q_{1}^{\bar{X}}, p_{1}^{X}, p_{2}^{X}, q_{2}^{\bar{X}}, p_{3}^{X}, q_{3}^{\bar{X}}\right), \\
& \mathcal{A}_{6}^{(0)}\left(q_{1}^{\bar{Y}}, p_{2}^{X}, p_{1}^{Y}, q_{2}^{\bar{X}}, q_{3}^{\bar{X}}, p_{3}^{X}\right) \\
& =\frac{s_{p_{3} q_{1}}}{s_{p_{1} p_{2} q_{2}} s_{p_{3} q_{3}}}-\frac{s_{p_{3} q_{2}}}{s_{p_{1} p_{2} q_{1}} s_{p_{3} q_{3}}}+\frac{1}{s_{p_{3} q_{1} q_{3}}}-\frac{1}{s_{p_{3} q_{2} q_{3}}} .
\end{aligned}
$$

Other amplitudes can be obtained by the SU(2) Super-Ward Identities and KK relations.

\section{One-loop BPS and SU(2) form factors}

In this appendix we discuss one-loop full-color BPS and SU(2) form factors, which are used in the two-loop subtraction. Besides, one loop analysis is also a simple test ground of the full-color method that we have developed.

From the analysis in section 3.2, one loop minimal loop corrections have only two basis given in (3.28):

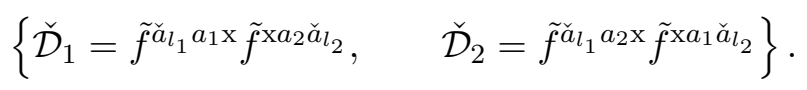

Also, one just needs planar matrix elements to reconstruct $\left[\mathcal{I}^{(1)}(12)\right]_{\check{D}_{i}}$. The required 


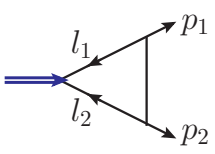

(a)

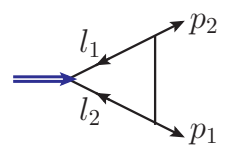

(b)

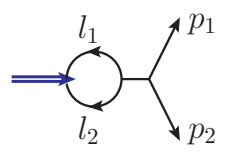

(c)

Figure 18. Trivalent topologies for one-loop non-BPS form factors. Note that we treat two triangle topology (a) and (b) differently, because the fields related to $l_{1}, l_{2}, p_{1}, p_{2}$ may be distinct for general form factor and an overall tree-level factors may not be factorized out.

planar elements can be given explicitly as:

$$
\begin{aligned}
& \left(\mathcal{I}^{(1), \mathrm{PL}}(12)\right)_{X X}^{X X}=-\mathrm{I}_{\text {tri }}\left[s_{12}\right] \\
& \left(\mathcal{I}^{(1), \mathrm{PL}}(12)\right)_{X Y}^{X Y}=-\mathrm{I}_{\text {tri }}\left[s_{12}\right]-\mathrm{I}_{\mathrm{bub}, 12}[1], \quad\left(\mathcal{I}^{(1), \mathrm{PL}}(12)\right)_{X Y}^{Y X}=\mathrm{I}_{\mathrm{bub}, 12}[1] .
\end{aligned}
$$

It is straightforward to use a double cut to fix these integands [36]. By dressing the color factors, the full-color loop correction density can be given as

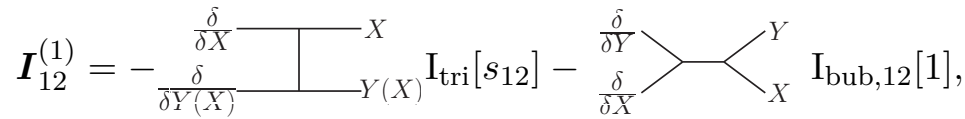

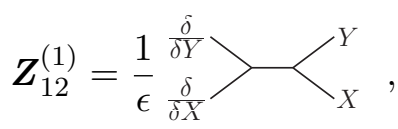

where $\frac{\delta}{\delta Y(X)}$ means variation acting on $\mathrm{Y}$ fields or X fields. Eq. (D.2) contains both oneloop BPS and SU(2) loop corrections. We also call the first part in $\boldsymbol{I}$ as $\boldsymbol{I}_{\text {tri }}$ and the second part $\boldsymbol{I}_{\text {bub }}$.

As mentioned in section 6, a CK-dual form for one-loop SU(2) form factor can also be constructed. One difference between the BPS and SU(2) case is that we have to distinguish external particles in the latter situation. As a result, the diagrams in figure 10 have to be extended to figure 18. With this fact in mind, we simply write down

$$
\boldsymbol{I}_{12, \mathrm{SU}(2)}^{(1)}=\tilde{f}^{\check{a}_{l_{1}} a_{1} \mathrm{x}_{1}} \tilde{f}^{\check{a}_{l_{2}} a_{2} \mathrm{x}_{1}} s_{2 l_{1}}=\underbrace{l_{1}}_{l_{2}} \overbrace{p_{2}}^{p_{1}}+\tilde{f}^{a_{l_{1}} a_{2} \mathrm{x}_{1}} \tilde{f}_{\tilde{a}_{l_{2}} a_{1} \mathrm{x}_{1}} s_{2 l_{1}}
$$

The CK duality check is straight forward: we have $N_{\text {tri,a }}=N_{\text {tri,b }}=s_{2 l_{1}}$ and $N_{\text {bub }}=0$. This fact ( $N_{\text {tri,a }}=N_{\text {tri,b }}$ and $\left.N_{\text {bub }}=0\right)$ can be generalized to more general form factors, usually including non-local numerator, i.e. numerator with $s_{12}$ pole. More concrete discussion will be covered in another article.

From the above discussion, one loop correction and renormalization constant have a very nice form which will be helpful for our understanding and calculation of the production of one-loop quantities in (7.41). For example, to get densities of $\left(\boldsymbol{Z}^{(1)}\right)^{2}$, one simply 
connects two $\boldsymbol{Z}^{(1)}$ together

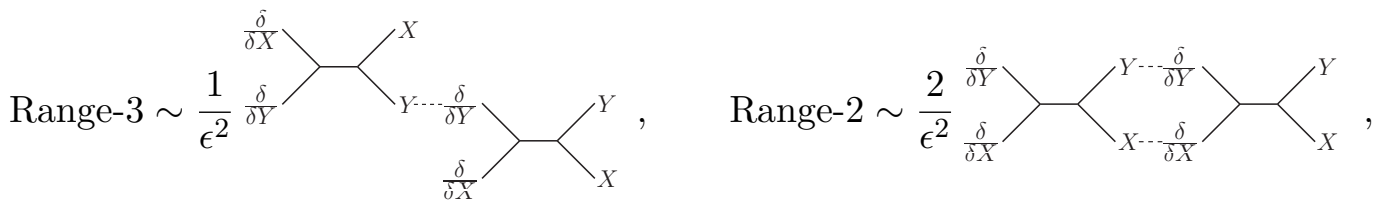

where the factor 2 in range- 2 density comes from permuting two internal contracted fields.

As for integrals appearing above, the one loop triangle integral is

$$
\mathrm{I}_{\text {tri, }, 12}[1]=\left(-s_{12}\right)^{-\epsilon-1}\left(-\frac{1}{\epsilon^{2}}+\frac{\zeta_{2}}{2}+O(\epsilon)\right) .
$$

And the one loop bubble integral is

$$
\mathrm{I}_{\mathrm{bub}, 12}[1]=\left(-s_{12}\right)^{-\epsilon}\left[\frac{1}{\epsilon}+2+\mathcal{O}(\epsilon)\right],
$$

where our convention of integrals is

$$
\mathrm{I}^{(L)}\left[N\left(l_{i}, p_{j}\right)\right]=e^{L \epsilon \gamma_{\mathrm{E}}} \int \frac{d^{D} l_{1}}{i \pi^{\frac{D}{2}}} \cdots \frac{d^{D} l_{L}}{i \pi^{\frac{D}{2}}} \frac{N\left(l_{i}, p_{j}\right)}{\prod_{j} D_{j}} .
$$

For completeness, two fish integrals used in section 7.3 are

$$
\begin{aligned}
\mathcal{I}_{\mathrm{SU}(2), \mathrm{b}}^{(2)}\left(p_{1}, p_{2}\right) & =\left(-s_{12}\right)^{-2 \epsilon}\left[\frac{1}{2 \epsilon^{2}}+\frac{5}{2 \epsilon}+\frac{19}{2}-\frac{\pi^{2}}{12}+\mathcal{O}(\epsilon)\right], \\
\mathcal{I}_{\mathrm{SU}(2), \mathrm{a}}^{(2)}\left(p_{1}, p_{2}, p_{3}\right) & =\left(-s_{12}\right)^{-2 \epsilon}\left[\frac{1}{2 \epsilon^{2}}+\frac{5}{2 \epsilon}+\frac{19}{2}-\frac{\pi^{2}}{12}-\left(\mathrm{Li}_{2}(1-u)+\frac{1}{2} \log ^{2}(u)\right)+\mathcal{O}(\epsilon)\right] .
\end{aligned}
$$

\section{E Cancellation of two-loop non-planar integrals}

In this appendix we give a proof to (5.38), following a similar discussion in [86].

To derive (5.38), we need a useful relation, which implies an implicit parity symmetry:

$$
\mathcal{I}_{3, \mathrm{np}}^{\prime}=\mathcal{I}_{3, \mathrm{np}},
$$

where

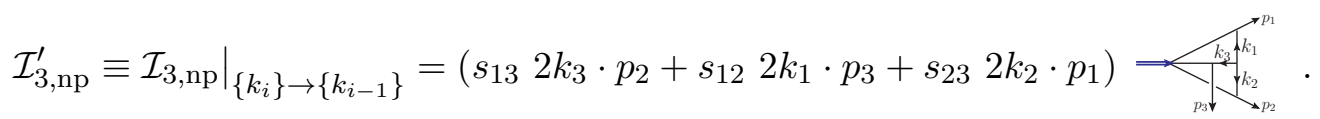

To prove this, we calculate $\Delta \mathcal{I}_{3, \mathrm{np}}=\mathcal{I}_{3, \mathrm{np}}-\mathcal{I}_{3, \mathrm{np}}^{\prime}$ with a new parametrization:

$$
\Delta \mathcal{I}_{3, \mathrm{np}}=e^{2 \epsilon \gamma_{E}} \int \frac{\mathrm{d}^{D} k_{1}}{i \pi^{\frac{D}{2}}} \frac{\mathrm{d}^{D} k_{2}}{i \pi^{\frac{D}{2}}} \frac{s_{13} 2\left(2 k_{1}+k_{2}\right) \cdot p_{2}+s_{12} 2\left(k_{2}-k_{1}\right) \cdot p_{3}-s_{23} 2\left(2 k_{2}+k_{1}\right) \cdot p_{1}}{k_{1}^{2} k_{2}^{2} k_{3}^{2}\left(k_{1}-p_{1}\right)^{2}\left(k_{2}-p_{2}\right)^{2}\left(k_{3}-p_{3}\right)^{2}} .
$$

Follow the discussion in [86], we write down

$$
k_{i}^{\mu}=\frac{p_{1}^{\mu}}{p_{1} \cdot p_{2}}\left(p_{2} \cdot k_{i}\right)+\frac{p_{2}^{\mu}}{p_{1} \cdot p_{2}}\left(p_{1} \cdot k_{i}\right)+k_{i, T},
$$


and define

$$
\xi_{i}=\frac{p_{1} \cdot p_{3}}{p_{1} \cdot p_{2}}\left(p_{2} \cdot k_{i}\right), \quad \eta_{i}=\frac{p_{2} \cdot p_{3}}{p_{1} \cdot p_{2}}\left(p_{1} \cdot k_{i}\right) .
$$

With these variables, we can re-write measure as

$$
\begin{aligned}
\mathrm{d}^{D} k_{i} & =\mathrm{d} k_{i}^{+} \mathrm{d} k_{i}^{-} \mathrm{d}^{D-2} k_{i, T} \\
& =\frac{p_{1} \cdot p_{2}}{\left(p_{1} \cdot p_{3}\right)\left(p_{2} \cdot p_{3}\right)} d \xi_{i} d \eta_{i} \mathrm{~d}^{D-2} k_{i, T},
\end{aligned}
$$

and

$$
k_{i}^{2}=2 \frac{\left(p_{1} \cdot k_{i}\right)\left(p_{2} \cdot k_{i}\right)}{p_{1} \cdot p_{2}}-k_{i, T}^{2}=2 \frac{p_{1} \cdot p_{2}}{\left(p_{1} \cdot p_{3}\right)\left(p_{2} \cdot p_{3}\right)} \xi_{i} \eta_{i}-k_{i, T}^{2} .
$$

If we exchange $\xi_{1} \leftrightarrow \eta_{2}, \xi_{2} \leftrightarrow \eta_{1}$ and $k_{1, T} \leftrightarrow k_{2, T}$, then $\left(k_{1}+p_{1}\right)^{2} \leftrightarrow\left(k_{2}+p_{2}\right)^{2}$ and $k_{1}^{2} \leftrightarrow k_{2}^{2}$. The denominator of (E.3) is symmetric under $\xi_{1} \leftrightarrow \eta_{2}, \xi_{2} \leftrightarrow \eta_{1}$ and $k_{1, T} \leftrightarrow k_{2, T}$. And the numerator, which can be written as

$$
\begin{aligned}
s_{13} 2\left(2 k_{1}\right. & \left.+k_{2}\right) \cdot p_{2}+s_{12} 2\left(k_{2}-k_{1}\right) \cdot p_{3}-s_{23} 2\left(2 k_{2}+k_{1}\right) \cdot p_{1} \\
& =\xi_{1}-\xi_{2}+\eta_{1}-\eta_{2}-p_{3, T} \cdot\left(k_{1}-k_{2}\right)_{T}+\xi_{1}+2 \xi_{2}-2 \eta_{1}-\eta_{2} .
\end{aligned}
$$

It is anti-symmetric under the transformation. So actually (E.3) gives zero. Namely, $\mathcal{I}_{3, \mathrm{np}}=\mathcal{I}_{3, n p}^{\prime}=\frac{\mathcal{I}_{3, \mathrm{np}}+\mathcal{I}_{3, n p}^{\prime}}{2}$ which equals to the following form using momentum conservation

$$
-\left(s_{13} k_{2} \cdot p_{2}+s_{12} k_{3} \cdot p_{3}+s_{23} k_{1} \cdot p_{1}\right) \underset{p_{3} r_{p_{2}}^{k_{3}} k_{k_{1}}^{p_{1}}}{k_{k_{2}}^{p_{2}}} .
$$

\section{F Details for IR and UV cancellation}

In this appendix we provide some details of IR and UV subtractions for two-loop form factors.

\section{F.1 Details for divergence cancellation in (7.8)}

Performing subtractions in (7.8) requires a full-color generalization of production of oneloop quantities, such as the one-loop-square. To fully illustrate the full-color one-loopsquare, we take a little detour and consider a general case. Concretely, we consider two arbitrary one-loop quantities $\boldsymbol{P}^{(1)}$ and $\boldsymbol{Q}^{(1)}$, with color decomposition $\boldsymbol{P}^{(1)}=\sum_{\sigma \in S_{2}} \sigma$. $\left(\tilde{f}^{\check{a}_{l_{1}} a_{1} \mathrm{x}} \tilde{f}^{\mathrm{x} a_{2} \check{a}_{l_{2}}} \mathcal{P}^{(1)}\right)$, and their product $\boldsymbol{P}^{(1)} \boldsymbol{Q}^{(1)}$. To dress the one loop product with color, we impose the condition that the planar component of full-color one-loop square should recover planar results, because it has been proven to be effective in the planar case. It turns out that the product reads ${ }^{25}$

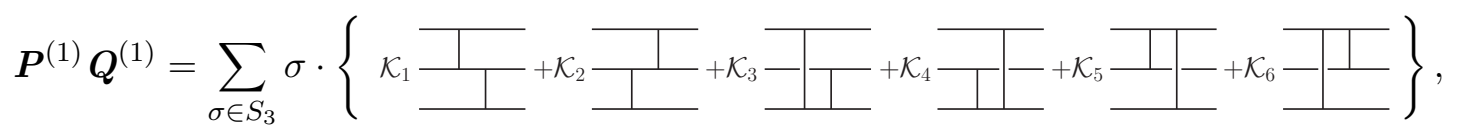

\footnotetext{
${ }^{25}$ The permutation $\sigma$ act on all the external labels, including momenta, color indices and the field configurations.
} 
where we omit the labels for color factors represented by graphs

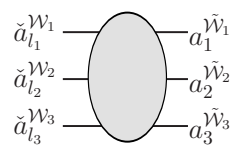

inside the gray oval can be any color factors in (F.1).

The kinematic factors in (F.1) are

$$
\begin{aligned}
& \mathcal{K}_{1}=\left(\mathcal{P}^{(1)}\right)_{\mathcal{W}_{I} \mathcal{W}_{3}}^{\widetilde{\mathcal{W}}_{2} \widetilde{\mathcal{W}}_{3}}\left(p_{2}, p_{3}\right)\left(\mathcal{Q}^{(1)}\right)_{\mathcal{W}_{1} \mathcal{W}_{2}}^{\widetilde{\mathcal{W}}_{1} \mathcal{W}_{I}}\left(p_{1}, p_{2}\right), \\
& \mathcal{K}_{2}=\left(\mathcal{Q}^{(1)}\right)_{\mathcal{W}_{2} \mathcal{W}_{3}}^{\mathcal{W}_{1} \widetilde{\mathcal{W}}_{3}}\left(p_{2}, p_{3}\right)\left(\mathcal{P}^{(1)}\right)_{\mathcal{W}_{1} \mathcal{W}_{2}}^{\widetilde{\mathcal{W}}_{1} \widetilde{\mathcal{W}}_{2}}\left(p_{1}, p_{2}\right), \\
& \mathcal{K}_{3}=\left(\mathcal{P}^{(1)}\right)_{\mathcal{W}_{2} \mathcal{W}_{I}}^{\widetilde{\mathcal{W}}_{2} \widetilde{\mathcal{W}}_{3}}\left(p_{2}, p_{3}\right)\left(\mathcal{Q}^{(1)}\right)_{\mathcal{W}_{1} \mathcal{W}_{3}}^{\widetilde{\mathcal{W}}_{1} \mathcal{W}_{I}}\left(p_{1}, p_{3}\right), \\
& \mathcal{K}_{4}=\left(\mathcal{Q}^{(1)}\right)_{\mathcal{W}_{2} \mathcal{W}_{3}}^{\widetilde{\mathcal{W}}_{2} \mathcal{W}_{I}}\left(p_{2}, p_{3}\right)\left(\mathcal{P}^{(1)}\right)_{\mathcal{W}_{1} \mathcal{W}_{I}}^{\widetilde{\mathcal{W}}_{1} \widetilde{\mathcal{W}}_{3}}\left(p_{1}, p_{3}\right), \\
& \mathcal{K}_{5}=\left(\mathcal{P}^{(1)}\right)_{\mathcal{W}_{I} \mathcal{W}_{3}}^{\widetilde{\mathcal{W}}_{1} \widetilde{\mathcal{W}}_{3}}\left(p_{1}, p_{3}\right)\left(\mathcal{Q}^{(1)}\right)_{\mathcal{W}_{1} \mathcal{W}_{2}}^{\mathcal{W}_{I} \widetilde{\mathcal{W}}_{2}}\left(p_{1}, p_{2}\right), \\
& \mathcal{K}_{6}=\left(\mathcal{Q}^{(1)}\right)_{\mathcal{W}_{1} \mathcal{W}_{3}}^{\mathcal{W}_{I} \widetilde{\mathcal{W}}_{3}}\left(p_{1}, p_{3}\right)\left(\mathcal{P}^{(1)}\right)_{\mathcal{W}_{I} \mathcal{W}_{2}}^{\widetilde{\mathcal{W}}_{1} \widetilde{\mathcal{W}}_{2}}\left(p_{1}, p_{2}\right),
\end{aligned}
$$

where $\mathcal{W}_{I}$ is intermediate fields: for our discussion for $\mathrm{SU}(2)$ form factors, it can be $X$ or $Y$ according to the specific situation.

As an example of (F.2), one can take $\boldsymbol{H}^{(2)}$ function as a commutator, whose origination is discussed in (7.60):

$$
\begin{aligned}
& \boldsymbol{H}_{123}^{(2)}=\frac{1}{2}\left(\left.\left.\boldsymbol{I}_{23}^{(1)}\right|_{\text {fin }} \boldsymbol{I}_{12}^{(1)}\right|_{\text {div }}-\left.\left.\boldsymbol{I}_{12}^{(1)}\right|_{\text {fin }} \boldsymbol{I}_{23}^{(1)}\right|_{\text {div }}\right)+\operatorname{cyc}\left(p_{1}, p_{2}, p_{3}\right)
\end{aligned}
$$

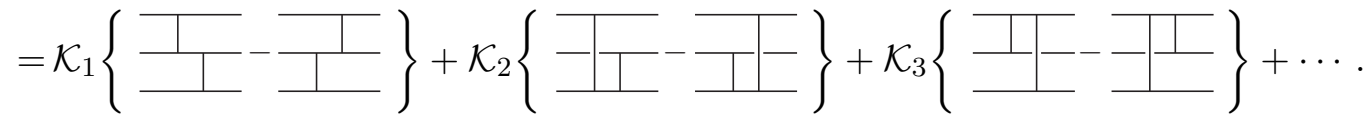

In this case, we take the difference between $\boldsymbol{P}=\left.\boldsymbol{I}\right|_{\mathrm{fin}}, \boldsymbol{Q}=\left.\boldsymbol{I}\right|_{\mathrm{div}}$ and $\boldsymbol{Q}=\left.\boldsymbol{I}\right|_{\mathrm{fin}}, \boldsymbol{P}=\left.\boldsymbol{I}\right|_{\mathrm{div}}$, i.e. the commutator. This example clearly shows that the order in the full-color problem is extremely important and must be paid extra attention to. Below we only explicitly list terms contribute to $\check{\mathcal{D}}_{19}$ and the label of external lines of color diagrams are always 123 in these terms. The $\mathcal{K}_{i}$ are

$$
\begin{aligned}
& \mathcal{K}_{1}=\left.\left.\frac{1}{2} \mathcal{I}^{(1)}(12)\right|_{\text {div }} \mathcal{I}^{(1)}(23)\right|_{\text {fin }}-\left.\left.\frac{1}{2} \mathcal{I}^{(1)}(23)\right|_{\text {div }} \mathcal{I}^{(1)}(12)\right|_{\text {fin }}, \\
& \mathcal{K}_{2}=\left.\left.\frac{1}{2} \mathcal{I}^{(1)}(23)\right|_{\text {div }} \mathcal{I}^{(1)}(13)\right|_{\text {fin }}-\left.\left.\frac{1}{2} \mathcal{I}^{(1)}(13)\right|_{\text {div }} \mathcal{I}^{(1)}(23)\right|_{\text {fin }}, \\
& \mathcal{K}_{3}=\left.\left.\frac{1}{2} \mathcal{I}^{(1)}(13)\right|_{\text {div }} \mathcal{I}^{(1)}(12)\right|_{\text {fin }}-\left.\left.\frac{1}{2} \mathcal{I}^{(1)}(12)\right|_{\text {div }} \mathcal{I}^{(1)}(13)\right|_{\text {fin }}
\end{aligned}
$$

Interestingly, all of the color-coefficient of $\mathcal{K}_{i}$ is $-\check{\mathcal{D}}_{19}$, leading to

$$
\left[\mathcal{H}^{(2)}\right]_{\check{\mathcal{D}}_{19}}(123)=-\sum_{i=1}^{3} \mathcal{K}_{i}=\frac{1}{4 \epsilon} \log \left(\frac{-s_{12}}{-s_{23}}\right) \log \left(\frac{-s_{23}}{-s_{13}}\right) \log \left(\frac{-s_{13}}{-s_{12}}\right) .
$$

Now we can say that the production of one-loop quantities is clear, and the derivation of one-loop-square in (7.13) and (7.14) is straightforward. 
Afterwards, we can perform subtractions and define remainders as in section 7.2. Recall that to define remainder at density level, we introduce auxiliary divergences $U$ which do not appear in the IR subtraction formula (7.8). So we check the cancellation of these $U$ s. Collecting all the $U$ together, we have

$$
\begin{aligned}
\boldsymbol{U}= & \sum_{i<j<k}\left\{\sum_{\substack{\sigma^{i} \in \mathbb{Z}_{3} \\
\sigma^{e} \in S_{3}}} \check{\mathcal{D}}_{\Delta_{1}}\left(\sigma^{i}, \sigma^{e}\right)\left(\sigma^{e} \cdot(U(i j)+U(j k))\right)\right. \\
& \left.-\sum_{\sigma^{e} \in S_{3}} \check{\mathcal{D}}_{\Delta_{2}}\left(\sigma^{e}\right)(U(i j)+U(j k)+U(i k))\right\} \\
& +2 \sum_{i<j} \sum_{\sigma^{e} \in S_{2}}\left(\check{\mathcal{D}}_{\Delta_{3}}\left(\sigma^{e}\right) U(i j)-2 \check{\mathcal{D}}_{\Delta_{4}}(\mathbf{1}) U(i j)\right) .
\end{aligned}
$$

$\boldsymbol{U}$ has the similar structure as (6.26) and it is easy to check the following identity beyond density level

$$
\boldsymbol{U} \cdot \boldsymbol{F}^{(0)}=0, \quad \forall \boldsymbol{F}^{(0)} .
$$

Finally, we also comment on the $N_{c}$ factor in the definition of $\tilde{f}^{(2)}$. Such a factor is natural to expect because we use coupling $g_{Y M}^{2}$ here rather than $g_{Y M}^{2} N_{c}$ (in the planar calculation). In our definition of $\left[\mathcal{R}^{(2)}\right]_{\check{\mathcal{D}}_{i}}$ with $i=25,26,27$ in (7.28) and (7.32), we already include a $\tilde{f}^{(2)}$ factor, namely, we have expand the term $\tilde{f}^{(2)} \boldsymbol{I}^{(1)}(2 \epsilon)$ in (7.8) with two-loop range- 2 color basis. More precisely, we have used the following color identity

$$
\check{\mathcal{D}}_{25}+\check{\mathcal{D}}_{26}+2 \check{\mathcal{D}}_{27}=2 N_{c} f^{\check{a}_{l_{1}} a_{1} \mathrm{x}_{1}} f^{\mathrm{x}_{1} a_{2} \check{a}_{l_{2}},}
$$

where $f^{\check{a}_{l_{1}} a_{1} \mathrm{x}_{1}} f^{\mathrm{x}_{1} a_{2} \check{a}_{l_{2}}}$ is the color factor of full-color one-loop density $\boldsymbol{I}^{(1)}$. This explains the $f^{(2)}$ term, for example, in (7.28).

\section{F.2 Analysis for SU(2) form factors}

Below we provide further details for the $\mathrm{SU}(2)$ form factors. We first reorganize the twoloop results in a simpler form, and then discuss the cancellation of divergences.

Simplified full-color density. The SU(2) density function $\boldsymbol{I}$ given in section 5.3 can be simplified if we choose another set of basis, which is motivated by rendering various symmetry manifest.

First we observe that $\tilde{\boldsymbol{I}}_{\mathrm{SU}(2)}$ (as defined in (5.53)) should be symmetric if exchanging two internal or external $X$ fields. So we have ${ }^{26}$

$$
\begin{aligned}
& \tilde{\boldsymbol{I}}_{\mathrm{SU}(2)}^{(2), r=3}\left(p_{1}^{Y}, p_{2}^{X}, p_{3}^{X}\right)=\left(\sum_{\sigma_{a} \in \mathbb{Z}_{3}} \sum_{\sigma_{b} \in \mathbb{Z}_{3}}\left\{\begin{array}{l}
\sigma_{b}\left(\check{a}_{h}\right)-\omega_{b} \\
\sigma_{b}\left(\tilde{a}_{2}\right)-\sigma_{a}\left(a_{1}\right) \\
\sigma_{b}\left(\tilde{l}_{3}\right)-\sigma_{a}\left(a_{2}\right)
\end{array}\left(\tilde{\mathcal{I}}_{\mathrm{SU}(2)}\right)_{\sigma_{b}\left(a_{3}\right)}^{\sigma_{a}(Y X X)}\left(\sigma_{a}\left(p_{1}, p_{2}, p_{3}\right)\right)\right\}\right. \\
& \left.+\left(\check{a}_{l_{2}}^{X} \leftrightarrow \check{a}_{l_{3}}^{X}\right)\right)+\left(a_{2}, p_{2} \leftrightarrow a_{3}, p_{3}\right),
\end{aligned}
$$

leaving us 9 functions $\left(\tilde{\mathcal{I}}_{\mathrm{SU}(2)}\right)_{\sigma_{b}(Y X X)}^{\sigma_{a}(Y X X)}$.

\footnotetext{
${ }^{26}$ Note that although the form has better symmetric property, the color basis for range-3 density are not all independent, providing us freedom for adjusting the kinematic parts which we use below.
} 
Second, we still have $\mathrm{SU}(2)$ relations satisfied by these functions

$$
\sum_{\tau_{b}}\left(\tilde{\mathcal{I}}_{\mathrm{SU}(2)}\right)_{\tau_{b}(Y X X)}^{\tau_{a}(Y X X)}=0, \quad \forall \tau_{a} \in S_{3} ; \quad \sum_{\tau_{a}}\left(\tilde{\mathcal{I}}_{\mathrm{SU}(2)}\right)_{\tau_{b}(Y X X)}^{\tau_{a}(Y X X)}=0, \quad \forall \tau_{b} \in Z_{3} .
$$

These SU(2) relations are identities at the function level and the difference between permutation $\tau$ and $\sigma$ can be found in section 5.3. These six equations are not independent and from them one finds four basis functions for $\tilde{\mathcal{I}}_{\mathrm{SU}(2)}$

$$
\mathcal{B}_{1}=\left(\tilde{\mathcal{I}}_{\mathrm{SU}(2)}\right)_{Y X X}^{Y X X}, \mathcal{B}_{2}=\left(\tilde{\mathcal{I}}_{\mathrm{SU}(2)}\right)_{Y X X}^{X X Y}, \mathcal{B}_{3}=\left(\tilde{\mathcal{I}}_{\mathrm{SU}(2)}\right)_{X X Y}^{Y X X}, \mathcal{B}_{4}=\left(\tilde{\mathcal{I}}_{\mathrm{SU}(2)}\right)_{X X Y}^{X X Y} .
$$

One can further set $\mathcal{B}_{3}=\mathcal{B}_{4}=0$ in (F.10). At this stage, this is nothing but a choice and we will explain this choice later. Other non-vanishing kinematic matrix elements are

$$
\begin{array}{ll}
\left(\tilde{\mathcal{I}}_{\mathrm{SU}(2)}\right)_{Y X X}^{X Y X}=-\mathcal{B}_{1}, & \left(\tilde{\mathcal{I}}_{\mathrm{SU}(2)}\right)_{X Y X}^{Y X X}=-\mathcal{B}_{1}-\mathcal{B}_{2}, \\
\left(\tilde{\mathcal{I}}_{\mathrm{SU}(2)}\right)_{X X Y}^{X Y X}=-\mathcal{B}_{2}, & \left(\tilde{\mathcal{I}}_{\mathrm{SU}(2)}\right)_{X Y X}^{X Y X}=\mathcal{B}_{1}+\mathcal{B}_{2} .
\end{array}
$$

Comparing this ansatz form (F.8) with the unitarity results obtained in section 5.3, we see that such basis functions are nothing but the integrals in section 7.3

$$
\mathcal{B}_{1}\left(p_{1}, p_{2}, p_{3}\right)=\mathcal{I}_{\mathrm{SU}(2), \mathrm{c}}^{(2)}\left(p_{1}, p_{2}, p_{3}\right), \quad \mathcal{B}_{2}\left(p_{1}, p_{2}, p_{3}\right)=\mathcal{I}_{\mathrm{SU}(2), \mathrm{a}}^{(2)}\left(p_{1}, p_{2}, p_{3}\right) .
$$

This also justifies the rationale of our previous choice about $\mathcal{B}_{3}=\mathcal{B}_{4}=0$.

Using (F.10) and (F.12), we can get (7.47) and (7.46), by collecting color factors for the same kinematic functions. For example, consider $\left(\tilde{\mathcal{I}}_{\mathrm{SU}(2)}\right)_{Y X X}^{X Y X}$ and $\left(\tilde{\mathcal{I}}_{\mathrm{SU}(2)}\right)_{Y X X}^{Y X X}$. They all have $\mathcal{B}_{1}\left(p_{1}, p_{2}, p_{3}\right)$ kinematic factor, and the color coefficients of such kinematic part are

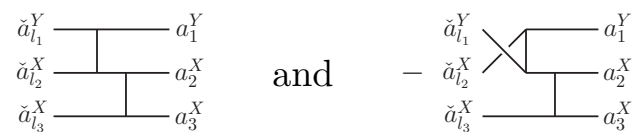

respectively. These two color factors satisfy

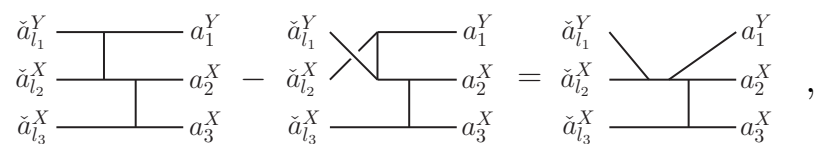

which recovers $\check{\mathcal{C}}_{\text {mix,A }}^{r=3}$ in $(7.47)$.

Finally, one can also consider a check with Feynman diagram computation. For

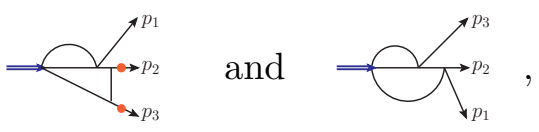

the color factors in (7.46) and (7.47) seem to be natural. The only remaining problem is that from Feynman diagrams, the color factor of

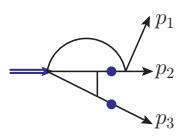


should look like

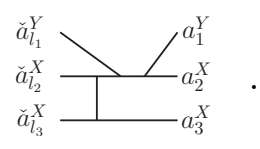

This problem can be resolved because one can generate $\check{\mathcal{C}}_{\text {mix }}^{r=3}$ using the identity in the following pattern

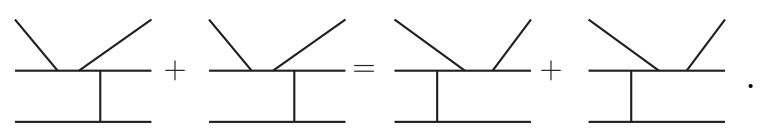

Details for divergence cancellation. The subtractions of $\mathrm{SU}(2)$ form factors in section 7.3 are separated into three parts. The UV part, i.e. $\boldsymbol{I}_{\text {fish }}$ contributing to the important dilatation operator, has been discussed in detail there. The subtractions and remainders of $\boldsymbol{I}_{\mathrm{BPS}}$ have no difference from the BPS subtraction in section 7.1 and 7.2. Here we provide some further discussions for $\boldsymbol{I}_{\mathrm{mix}}$.

One should be cautious about the difference between (7.45) and (7.41)|they are not strictly equivalent. The problem comes from the order of $\underline{\boldsymbol{I}}_{\text {bub }}^{(1)}$ and $\underline{\boldsymbol{I}}_{\mathrm{tri}}^{(1)}$. Directly from (7.41), the proper order of $\underline{\boldsymbol{I}}_{\text {tri }}^{(1)}$ and $\underline{\boldsymbol{I}}_{\text {bub }}^{(1)}$ is $\frac{1}{2}\left\{\underline{\boldsymbol{I}}_{\text {tri }}^{(1)}, \underline{\boldsymbol{I}}_{\text {bub }}^{(1)}\right\}$, which have a commutator $\frac{1}{2}\left[\underline{\boldsymbol{I}}_{\mathrm{tri}}^{(1)}, \underline{\boldsymbol{I}}_{\mathrm{bub}}^{(1)}\right]$ difference from $\underline{\boldsymbol{I}}_{\mathrm{tri}}^{(1)} \underline{\boldsymbol{I}}_{\mathrm{bub}}^{(1)}$ in $(7.45)$. This commutator, with $\epsilon^{-1}$ divergence, can be cancelled by $\boldsymbol{H}_{\text {mix }}^{(2)}$ discussed in section 7.4. Nevertheless, it is convenient to just write down the subtraction (7.45) and obtain the remainders in section 7.3.

After discussing the subtraction equation (7.45), the subtraction procedure is mostly depicted in section 7.3 with one small remaining problem, which is the cancellation of auxiliary divergence $V=\zeta_{2} / \epsilon$. Such an auxiliary quantity just shows up in subtractions of $\boldsymbol{I}_{\text {mix }}$, whose color factors (those $\check{C}_{\text {mix }}$ ) are presented in (7.48). For example, if the external states are $p_{1}^{Y}, p_{2}^{X}, p_{3}^{X}$, then the contributions, with color factors, proportional to $V=\zeta_{2} / \epsilon$ are

$$
\begin{aligned}
\boldsymbol{V}_{123} & =V\left(\check{\mathcal{C}}_{\text {mix }, \mathrm{A}}^{r=3}+\check{\mathcal{C}}_{\text {mix }, \mathrm{B}}^{r=3}+\left(a_{2} \leftrightarrow a_{3}\right)\right) \\
\boldsymbol{V}_{12} & =2 V \check{\mathcal{C}}_{\text {mix }}^{r=2}, \quad \check{V}_{13}=\left.2 V \check{\mathcal{C}}_{\text {mix }}^{r=2}\right|_{a_{2} \rightarrow a_{3}}
\end{aligned}
$$

Summing up all these auxiliary divergence, we find out that they cancel beyond density level

$$
\boldsymbol{V} \cdot \boldsymbol{F}^{(0)}=\left(\sum_{i<j<k} \boldsymbol{V}_{i j k}+\sum_{i<j} \boldsymbol{V}_{i j}\right) \cdot \boldsymbol{F}^{(0)}=0 .
$$

Note that both $\boldsymbol{V}_{i j k}$ and $\boldsymbol{F}^{(0)}$ depend on specific operators, so we have to analysis case by case. Checks performed up to length- 6 operators with arbitrary distribution of $X, Y$ support this cancellation. It would be interesting to have a general proof of such a conclusion.

Open Access. This article is distributed under the terms of the Creative Commons Attribution License (CC-BY 4.0), which permits any use, distribution and reproduction in any medium, provided the original author(s) and source are credited. 


\section{References}

[1] G. 't Hooft, A planar diagram theory for strong interactions, Nucl. Phys. B 72 (1974) 461 [INSPIRE].

[2] N. Beisert et al., Review of AdS/CFT integrability: an overview, Lett. Math. Phys. 99 (2012) 3 [arXiv: 1012.3982] [INSPIRE].

[3] W.L. van Neerven, Infrared behavior of on-shell form-factors in a $N=4$ supersymmetric Yang-Mills field Theory, Z. Phys. C 30 (1986) 595 [InSPIRE].

[4] L.F. Alday and J. Maldacena, Comments on gluon scattering amplitudes via AdS/CFT, JHEP 11 (2007) 068 [arXiv:0710.1060] [INSPIRE].

[5] J. Maldacena and A. Zhiboedov, Form factors at strong coupling via a Y-system, JHEP 11 (2010) 104 [arXiv: 1009.1139] [INSPIRE].

[6] A. Brandhuber, B. Spence, G. Travaglini and G. Yang, Form factors in $N=4$ super Yang-Mills and periodic Wilson loops, JHEP 01 (2011) 134 [arXiv:1011.1899] [INSPIRE].

[7] L.V. Bork, D.I. Kazakov and G.S. Vartanov, On form factors in N=4 SYM, JHEP 02 (2011) 063 [arXiv: 1011.2440] [INSPIRE].

[8] A. Brandhuber, O. Gurdogan, R. Mooney, G. Travaglini and G. Yang, Harmony of super form factors, JHEP 10 (2011) 046 [arXiv:1107.5067] [INSPIRE].

[9] L.V. Bork, D.I. Kazakov and G.S. Vartanov, On MHV form factors in superspace for $\mathcal{N}=4$ SYM theory, JHEP 10 (2011) 133 [arXiv:1107.5551] [INSPIRE].

[10] J.M. Henn, S. Moch and S.G. Naculich, Form factors and scattering amplitudes in $N=4$ SYM in dimensional and massive regularizations, JHEP 12 (2011) 024 [arXiv:1109.5057] [INSPIRE].

[11] A. Brandhuber, G. Travaglini and G. Yang, Analytic two-loop form factors in $N=4 S Y M$, JHEP 05 (2012) 082 [arXiv: 1201.4170] [INSPIRE].

[12] Z. Gao and G. Yang, Y-system for form factors at strong coupling in $A d S_{5}$ and with multi-operator insertions in $A d S_{3}$, JHEP 06 (2013) 105 [arXiv:1303.2668] [INSPIRE].

[13] B. Penante, B. Spence, G. Travaglini and C. Wen, On super form factors of half-BPS operators in $N=4$ super Yang-Mills, JHEP 04 (2014) 083 [arXiv:1402.1300] [INSPIRE].

[14] A. Brandhuber, B. Penante, G. Travaglini and C. Wen, The last of the simple remainders, JHEP 08 (2014) 100 [arXiv: 1406.1443] [INSPIRE].

[15] R. Frassek, D. Meidinger, D. Nandan and M. Wilhelm, On-shell diagrams, Graßmannians and integrability for form factors, JHEP 01 (2016) 182 [arXiv: 1506.08192] [INSPIRE].

[16] L.V. Bork and A.I. Onishchenko, Grassmannians and form factors with $q^{2}=0$ in $\mathcal{N}=4$ SYM theory, JHEP 12 (2016) 076 [arXiv:1607.00503] [INSPIRE].

[17] L.V. Bork and A.I. Onishchenko, Wilson lines, Grassmannians and gauge invariant off-shell amplitudes in $\mathcal{N}=4$ SYM, JHEP 04 (2017) 019 [arXiv:1607.02320] [INSPIRE].

[18] L.V. Bork and A.I. Onishchenko, Four dimensional ambitwistor strings and form factors of local and Wilson line operators, Phys. Rev. D 97 (2018) 126013 [arXiv:1704.04758] [INSPIRE].

[19] L.V. Bork, On form factors in $\mathcal{N}=4$ SYM theory and polytopes, JHEP 12 (2014) 111 [arXiv: 1407.5568] [INSPIRE]. 
[20] L. Koster, V. Mitev, M. Staudacher and M. Wilhelm, Composite operators in the twistor formulation of $N=4$ supersymmetric Yang-Mills theory, Phys. Rev. Lett. 117 (2016) 011601 [arXiv: 1603.04471] [INSPIRE].

[21] L. Koster, V. Mitev, M. Staudacher and M. Wilhelm, All tree-level MHV form factors in $\mathcal{N}=4$ SYM from twistor space, JHEP 06 (2016) 162 [arXiv:1604.00012] [INSPIRE].

[22] D. Chicherin and E. Sokatchev, Composite operators and form factors in $\mathcal{N}=4 S Y M, J$. Phys. A 50 (2017) 275402 [arXiv:1605.01386] [InSPIRE].

[23] L. Koster, V. Mitev, M. Staudacher and M. Wilhelm, On form factors and correlation functions in twistor space, JHEP 03 (2017) 131 [arXiv:1611.08599] [INSPIRE].

[24] S. He and Y. Zhang, Connected formulas for amplitudes in standard model, JHEP 03 (2017) 093 [arXiv: 1607.02843] [InSPIRE].

[25] A. Brandhuber, E. Hughes, R. Panerai, B. Spence and G. Travaglini, The connected prescription for form factors in twistor space, JHEP 11 (2016) 143 [arXiv:1608.03277] [INSPIRE].

[26] S. He and Z. Liu, A note on connected formula for form factors, JHEP 12 (2016) 006 [arXiv: 1608.04306] [INSPIRE].

[27] A.E. Bolshov, L.V. Bork and A.I. Onishchenko, The all-loop conjecture for integrands of reggeon amplitudes in $\mathcal{N}=4$ SYM, JHEP 06 (2018) 129 [arXiv: 1802.03986] [INSPIRE].

[28] L. Bianchi, A. Brandhuber, R. Panerai and G. Travaglini, Form factor recursion relations at loop level, JHEP 02 (2019) 182 [arXiv: 1812.09001] [INSPIRE].

[29] L. Bianchi, A. Brandhuber, R. Panerai and G. Travaglini, Dual conformal invariance for form factors, JHEP 02 (2019) 134 [arXiv: 1812.10468] [INSPIRE].

[30] L.V. Bork, On NMHV form factors in $N=4 S Y M$ theory from generalized unitarity, JHEP 01 (2013) 049 [arXiv: 1203.2596] [inSPIRE].

[31] H. Johansson, D.A. Kosower and K.J. Larsen, Two-loop maximal unitarity with external masses, Phys. Rev. D 87 (2013) 025030 [arXiv: 1208.1754] [INSPIRE].

[32] R. Huang, Q. Jin and B. Feng, Form factor and boundary contribution of amplitude, JHEP 06 (2016) 072 [arXiv:1601.06612] [InSPIRE].

[33] M. Accettulli Huber, A. Brandhuber, S. De Angelis and G. Travaglini, Complete form factors in Yang-Mills from unitarity and spinor helicity in six dimensions, Phys. Rev. D 101 (2020) 026004 [arXiv: 1910.04772] [INSPIRE].

[34] M. Wilhelm, Amplitudes, form factors and the dilatation operator in $\mathcal{N}=4 S Y M$ theory, JHEP 02 (2015) 149 [arXiv:1410.6309] [INSPIRE].

[35] D. Nandan, C. Sieg, M. Wilhelm and G. Yang, Cutting through form factors and cross sections of non-protected operators in $\mathcal{N}=4$ SYM, JHEP 06 (2015) 156 [arXiv: 1410.8485$]$ [INSPIRE].

[36] F. Loebbert, D. Nandan, C. Sieg, M. Wilhelm and G. Yang, On-shell methods for the two-loop dilatation operator and finite remainders, JHEP 10 (2015) 012 [arXiv: 1504.06323] [INSPIRE].

[37] A. Brandhuber, M. Kostacinska, B. Penante, G. Travaglini and D. Young, The SU(2|3) dynamic two-loop form factors, JHEP 08 (2016) 134 [arXiv:1606.08682] [INSPIRE]. 
[38] F. Loebbert, C. Sieg, M. Wilhelm and G. Yang, Two-loop SL(2) form factors and maximal transcendentality, JHEP 12 (2016) 090 [arXiv:1610.06567] [INSPIRE].

[39] T. Ahmed, P. Banerjee, P.K. Dhani, N. Rana, V. Ravindran and S. Seth, Konishi form factor at three loops in $\mathcal{N}=4$ supersymmetric Yang-Mills theory, Phys. Rev. D 95 (2017) 085019 [arXiv: 1610.05317] [INSPIRE].

[40] A. Brandhuber, M. Kostacinska, B. Penante and G. Travaglini, Higgs amplitudes from $\mathcal{N}=4$ super Yang-Mills theory, Phys. Rev. Lett. 119 (2017) 161601 [arXiv:1707.09897] [INSPIRE].

[41] A. Brandhuber, M. Kostacinska, B. Penante and G. Travaglini, $\operatorname{Tr}\left(F^{3}\right)$ supersymmetric form factors and maximal transcendentality. Part I. $\mathcal{N}=4$ super Yang-Mills, JHEP 12 (2018) 076 [arXiv: 1804.05703] [INSPIRE].

[42] S. Caron-Huot and M. Wilhelm, Renormalization group coefficients and the S-matrix, JHEP 12 (2016) 010 [arXiv: 1607.06448] [INSPIRE].

[43] T. Ahmed and P.K. Dhani, Two-loop doubly massive four-point amplitude involving a half-BPS and Konishi operator, JHEP 05 (2019) 066 [arXiv:1901.08086] [INSPIRE].

[44] T. Ahmed, P. Banerjee, A. Chakraborty, P.K. Dhani and V. Ravindran, Form factors with two operator insertions and the principle of maximal transcendentality, Phys. Rev. D 102 (2020) 061701 [arXiv:1911.11886] [INSPIRE].

[45] G. Yang, On-shell methods for form factors in $\mathcal{N}=4 S Y M$ and their applications, Sci. China Phys. Mech. Astron. 63 (2020) 270001 [arXiv:1912.11454] [InSPIRE].

[46] R.H. Boels, B.A. Kniehl, O.V. Tarasov and G. Yang, Color-kinematic Duality for Form Factors, JHEP 02 (2013) 063 [arXiv: 1211.7028] [INSPIRE].

[47] G. Yang, Color-kinematics duality and Sudakov form factor at five loops for $N=4$ supersymmetric Yang-Mills theory, Phys. Rev. Lett. 117 (2016) 271602 [arXiv:1610.02394] [INSPIRE].

[48] Z. Bern, J.J.M. Carrasco and H. Johansson, New relations for gauge-theory amplitudes, Phys. Rev. D 78 (2008) 085011 [arXiv:0805.3993] [InSPIRE].

[49] Z. Bern, J.J.M. Carrasco and H. Johansson, Perturbative quantum gravity as a double copy of gauge theory, Phys. Rev. Lett. 105 (2010) 061602 [arXiv:1004.0476] [INSPIRE].

[50] A.C. Edison and S.G. Naculich, $\mathrm{SU}(N)$ group-theory constraints on color-ordered five-point amplitudes at all loop orders, Nucl. Phys. B 858 (2012) 488 [arXiv:1111.3821] [InSPIRE].

[51] A.C. Edison and S.G. Naculich, Symmetric-group decomposition of SU(N) group-theory constraints on four-, five-, and six-point color-ordered amplitudes, JHEP 09 (2012) 069 [arXiv:1207.5511] [INSPIRE].

[52] B. Feng, Y. Jia and R. Huang, Relations of loop partial amplitudes in gauge theory by Unitarity cut method, Nucl. Phys. B 854 (2012) 243 [arXiv:1105.0334] [INSPIRE].

[53] A. Ochirov and B. Page, Full colour for loop amplitudes in Yang-Mills theory, JHEP 02 (2017) 100 [arXiv:1612.04366] [INSPIRE].

[54] S. Abreu, L.J. Dixon, E. Herrmann, B. Page and M. Zeng, The two-loop five-point amplitude in $\mathcal{N}=4$ Super-Yang-Mills theory, Phys. Rev. Lett. 122 (2019) 121603 [arXiv: 1812.08941] [INSPIRE]. 
[55] S. Badger et al., Analytic form of the full two-loop five-gluon all-plus helicity amplitude, Phys. Rev. Lett. 123 (2019) 071601 [arXiv:1905.03733] [INSPIRE].

[56] A.R. Dalgleish, D.C. Dunbar, W.B. Perkins and J.M.W. Strong, Full color two-loop six-gluon all-plus helicity amplitude, Phys. Rev. D 101 (2020) 076024 [arXiv:2003.00897] [INSPIRE].

[57] Z. Bern, L.J. Dixon, D.C. Dunbar and D.A. Kosower, One loop $n$ point gauge theory amplitudes, unitarity and collinear limits, Nucl. Phys. B 425 (1994) 217 [hep-ph/9403226] [INSPIRE].

[58] Z. Bern, L.J. Dixon, D.C. Dunbar and D.A. Kosower, Fusing gauge theory tree amplitudes into loop amplitudes, Nucl. Phys. B 435 (1995) 59 [hep-ph/9409265] [INSPIRE].

[59] R. Britto, F. Cachazo and B. Feng, Generalized unitarity and one-loop amplitudes in $N=4$ Super-Yang-Mills, Nucl. Phys. B 725 (2005) 275 [hep-th/0412103] [INSPIRE].

[60] Z. Bern, J.J. Carrasco, M. Chiodaroli, H. Johansson and R. Roiban, The duality between color and kinematics and its applications, arXiv:1909.01358 [INSPIRE].

[61] T. Becher and M. Neubert, Infrared singularities of scattering amplitudes in perturbative QCD, Phys. Rev. Lett. 102 (2009) 162001 [Erratum ibid. 111 (2013) 199905] [arXiv:0901.0722] [INSPIRE].

[62] E. Gardi and L. Magnea, Factorization constraints for soft anomalous dimensions in QCD scattering amplitudes, JHEP 03 (2009) 079 [arXiv:0901.1091] [INSPIRE].

[63] N. Beisert, C. Kristjansen and M. Staudacher, The dilatation operator of conformal $N=4$ Super-Yang-Mills theory, Nucl. Phys. B 664 (2003) 131 [hep-th/0303060] [INSPIRE].

[64] V.P. Nair, A current algebra for some gauge theory amplitudes, Phys. Lett. B 214 (1988) 215.

[65] J.M. Henn and J.C. Plefka, Scattering amplitudes in gauge theories, Springer, Berlin Germany (2014).

[66] H. Elvang and Y.T. Huang, Scattering amplitudes in gauge theory and gravity, Cambridge University Press, Cambridge U.K. (2015).

[67] S.J. Parke and T.R. Taylor, An amplitude for $n$ gluon scattering, Phys. Rev. Lett. 56 (1986) 2459 [INSPIRE].

[68] R. Britto, F. Cachazo, B. Feng and E. Witten, Direct proof of tree-level recursion relation in Yang-Mills theory, Phys. Rev. Lett. 94 (2005) 181602 [hep-th/0501052] [INSPIRE].

[69] F. Cachazo, P. Svrček and E. Witten, MHV vertices and tree amplitudes in gauge theory, JHEP 09 (2004) 006 [hep-th/0403047] [INSPIRE].

[70] N. Beisert, On Yangian Symmetry in Planar $N=4 S Y M$, in the proceedings of Gribov-80 Memorial Workshop on Quantum Chromodynamics and Beyond, May 26-28, Trieste, Italy (2010), arXiv: 1004.5423 [INSPIRE].

[71] B.I. Zwiebel, From scattering amplitudes to the dilatation generator in $N=4 S Y M, J$. Phys. A 45 (2012) 115401 [arXiv:1111.0083] [InSPIRE].

[72] N. Beisert, The SU(2|3) dynamic spin chain, Nucl. Phys. B 682 (2004) 487 [hep-th/0310252] [INSPIRE].

[73] V. Del Duca, L.J. Dixon and F. Maltoni, New color decompositions for gauge amplitudes at tree and loop level, Nucl. Phys. B 571 (2000) 51 [hep-ph/9910563] [INSPIRE]. 
[74] R. Kleiss and H. Kuijf, Multi-gluon cross-sections and five jet production at hadron colliders, Nucl. Phys. B 312 (1989) 616 [inSPIRE].

[75] J.J.M. Carrasco and H. Johansson, Five-point amplitudes in $N=4$ Super-Yang-Mills theory and $N=8$ supergravity, Phys. Rev. D 85 (2012) 025006 [arXiv:1106.4711] [InSPIRE].

[76] Z. Bern, J.J.M. Carrasco, L.J. Dixon, H. Johansson and R. Roiban, Simplifying multiloop integrands and ultraviolet divergences of gauge theory and gravity amplitudes, Phys. Rev. D 85 (2012) 105014 [arXiv: 1201.5366] [INSPIRE].

[77] Z. Bern, S. Davies and T. Dennen, The ultraviolet structure of half-maximal supergravity with matter multiplets at two and three loops, Phys. Rev. D 88 (2013) 065007 [arXiv:1305.4876] [INSPIRE].

[78] Z. Bern, S. Davies and T. Dennen, Enhanced ultraviolet cancellations in $\mathcal{N}=5$ supergravity at four loops, Phys. Rev. D 90 (2014) 105011 [arXiv:1409.3089] [INSPIRE].

[79] Z. Bern, J.J. Carrasco, W.-M. Chen, H. Johansson and R. Roiban, Gravity amplitudes as generalized double copies of gauge-theory amplitudes, Phys. Rev. Lett. 118 (2017) 181602 [arXiv: 1701.02519] [INSPIRE].

[80] H. Johansson, G. Kälin and G. Mogull, Two-loop supersymmetric QCD and half-maximal supergravity amplitudes, JHEP 09 (2017) 019 [arXiv:1706.09381] [INSPIRE].

[81] Z. Bern, J.J.M. Carrasco, W.-M. Chen, H. Johansson, R. Roiban and M. Zeng, Five-loop four-point integrand of $N=8$ supergravity as a generalized double copy, Phys. Rev. D 96 (2017) 126012 [arXiv: 1708.06807] [inSPIRE].

[82] G. Kälin, G. Mogull and A. Ochirov, Two-loop $\mathcal{N}=2$ SQCD amplitudes with external matter from iterated cuts, JHEP 07 (2019) 120 [arXiv:1811.09604] [INSPIRE].

[83] R.H. Boels, R.S. Isermann, R. Monteiro and D. O'Connell, Colour-kinematics duality for one-loop rational amplitudes, JHEP 04 (2013) 107 [arXiv:1301.4165] [INSPIRE].

[84] Z. Bern, S. Davies, T. Dennen, Y.-t. Huang and J. Nohle, Color-kinematics duality for pure Yang-Mills and gravity at one and two loops, Phys. Rev. D 92 (2015) 045041 [arXiv: 1303.6605] [INSPIRE].

[85] G. Mogull and D. O'Connell, Overcoming obstacles to colour-kinematics duality at two loops, JHEP 12 (2015) 135 [arXiv:1511.06652] [INSPIRE].

[86] S.M. Aybat, L.J. Dixon and G.F. Sterman, The two-loop soft anomalous dimension matrix and resummation at next-to-next-to leading pole, Phys. Rev. D 74 (2006) 074004 [hep-ph/0607309] [INSPIRE].

[87] Z. Bern, L.J. Dixon and V.A. Smirnov, Iteration of planar amplitudes in maximally supersymmetric Yang-Mills theory at three loops and beyond, Phys. Rev. D 72 (2005) 085001 [hep-th/0505205] [INSPIRE].

[88] Z. Bern, A. De Freitas and L.J. Dixon, Two loop helicity amplitudes for gluon-gluon scattering in QCD and supersymmetric Yang-Mills theory, JHEP 03 (2002) 018 [hep-ph/0201161] [INSPIRE].

[89] Z. Bern, A. De Freitas and L.J. Dixon, Two loop helicity amplitudes for quark gluon scattering in QCD and gluino gluon scattering in supersymmetric Yang-Mills theory, JHEP 06 (2003) 028 [Erratum ibid. 04 (2014) 112] [hep-ph/0304168] [INSPIRE]. 
[90] Z. Bern, L.J. Dixon and D.A. Kosower, Two-loop $g \rightarrow$ gg splitting amplitudes in QCD, JHEP 08 (2004) 012 [hep-ph/0404293] [INSPIRE].

[91] A.H. Mueller, On the asymptotic behavior of the Sudakov form-factor, Phys. Rev. D 20 (1979) 2037 [INSPIRE].

[92] J.C. Collins, Algorithm to compute corrections to the Sudakov form-factor, Phys. Rev. D 22 (1980) 1478 [INSPIRE].

[93] A. Sen, Asymptotic behavior of the Sudakov form-Factor in QCD, Phys. Rev. D 24 (1981) 3281 [INSPIRE].

[94] L. Magnea and G.F. Sterman, Analytic continuation of the Sudakov form-factor in QCD, Phys. Rev. D 42 (1990) 4222 [INSPIRE].

[95] G.P. Korchemsky and A.V. Radyushkin, Loop space formalism and renormalization group for the infrared asymptotics of QCD, Phys. Lett. B 171 (1986) 459 [INSPIRE].

[96] G.P. Korchemsky, Asymptotics of the Altarelli-Parisi-Lipatov evolution kernels of parton distributions, Mod. Phys. Lett. A 4 (1989) 1257 [InSPIRE].

[97] F. Cachazo, M. Spradlin and A. Volovich, Four-loop collinear anomalous dimension in $N=4$ Yang-Mills theory, Phys. Rev. D 76 (2007) 106004 [arXiv:0707.1903] [INSPIRE].

[98] L.J. Dixon, The principle of maximal transcendentality and the four-loop collinear anomalous dimension, JHEP 01 (2018) 075 [arXiv:1712.07274] [INSPIRE].

[99] J.M. Henn, G.P. Korchemsky and B. Mistlberger, The full four-loop cusp anomalous dimension in $\mathcal{N}=4$ super Yang-Mills and QCD, JHEP 04 (2020) 018 [arXiv:1911.10174] [INSPIRE].

[100] T. Huber, A. von Manteuffel, E. Panzer, R.M. Schabinger and G. Yang, The four-loop cusp anomalous dimension from the $N=4$ Sudakov form factor, Phys. Lett. B 807 (2020) 135543 [arXiv: 1912.13459] [INSPIRE].

[101] R.H. Boels, T. Huber and G. Yang, Four-loop nonplanar cusp anomalous dimension in $N=4$ supersymmetric Yang-Mills theory, Phys. Rev. Lett. 119 (2017) 201601 [arXiv: 1705.03444] [INSPIRE].

[102] R.H. Boels, T. Huber and G. Yang, The Sudakov form factor at four loops in maximal super Yang-Mills theory, JHEP 01 (2018) 153 [arXiv:1711.08449] [INSPIRE].

[103] S. Catani, The singular behavior of QCD amplitudes at two loop order, Phys. Lett. B 427 (1998) 161 [hep-ph/9802439] [INSPIRE].

[104] G.F. Sterman and M.E. Tejeda-Yeomans, Multiloop amplitudes and resummation, Phys. Lett. B 552 (2003) 48 [hep-ph/0210130] [INSPIRE].

[105] A.V. Smirnov, Algorithm FIRE - Feynman Integral REduction, JHEP 10 (2008) 107 [arXiv:0807.3243] [INSPIRE].

[106] R.N. Lee, LiteRed 1.4: a powerful tool for reduction of multiloop integrals, J. Phys. Conf. Ser. 523 (2014) 012059 [arXiv: 1310.1145] [INSPIRE].

[107] P. Maierhöfer, J. Usovitsch and P. Uwer, Kira - A Feynman integral reduction program, Comput. Phys. Commun. 230 (2018) 99 [arXiv:1705.05610] [INSPIRE].

[108] T. Gehrmann and E. Remiddi, Two loop master integrals for $\gamma^{*} \rightarrow 3$ jets: the planar topologies, Nucl. Phys. B 601 (2001) 248 [hep-ph/0008287] [INSPIRE]. 
[109] T. Gehrmann and E. Remiddi, Numerical evaluation of two-dimensional harmonic polylogarithms, Comput. Phys. Commun. 144 (2002) 200 [hep-ph/0111255] [INSPIRE].

[110] A.B. Goncharov, M. Spradlin, C. Vergu and A. Volovich, Classical polylogarithms for amplitudes and Wilson loops, Phys. Rev. Lett. 105 (2010) 151605 [arXiv:1006.5703] [INSPIRE].

[111] A.B. Goncharov, A simple construction of Grassmannian polylogarithms, arXiv:0908.2238 [INSPIRE].

[112] A.V. Kotikov and L.N. Lipatov, DGLAP and BFKL equations in the $N=4$ supersymmetric gauge theory, Nucl. Phys. B 661 (2003) 19 [Erratum ibid. 685 (2004) 405] [hep-ph/0208220] [INSPIRE].

[113] A.V. Kotikov, L.N. Lipatov, A.I. Onishchenko and V.N. Velizhanin, Three loop universal anomalous dimension of the Wilson operators in $N=4$ SUSY Yang-Mills model, Phys. Lett. B 595 (2004) 521 [Erratum ibid. 632 (2006) 754] [hep-th/0404092] [INSPIRE].

[114] P. Banerjee, P.K. Dhani, M. Mahakhud, V. Ravindran and S. Seth, Finite remainders of the Konishi at two loops in $\mathcal{N}=4 S Y M$, JHEP 05 (2017) 085 [arXiv:1612.00885] [INSPIRE].

[115] P. Banerjee, P.K. Dhani and V. Ravindran, Two loop QCD corrections for the process Pseudo-scalar Higgs $\rightarrow 3$ partons, JHEP 10 (2017) 067 [arXiv: 1708.02387] [INSPIRE].

[116] Q. Jin and G. Yang, Analytic two-loop Higgs amplitudes in effective field theory and the maximal transcendentality principle, Phys. Rev. Lett. 121 (2018) 101603 [arXiv: 1804.04653] [INSPIRE].

[117] A. Brandhuber, M. Kostacinska, B. Penante and G. Travaglini, $\operatorname{Tr}\left(F^{3}\right)$ supersymmetric form factors and maximal transcendentality. Part II. $0<\mathcal{N}<4$ super Yang-Mills, JHEP 12 (2018) 077 [arXiv: 1804.05828] [INSPIRE].

[118] Q. Jin and G. Yang, Hidden analytic relations for two-loop Higgs amplitudes in QCD, Commun. Theor. Phys. 72 (2020) 065201 [arXiv: 1904.07260] [INSPIRE].

[119] Q. Jin and G. Yang, Two-loop QCD corrections to the Higgs plus three-parton amplitudes with top mass correction, JHEP 02 (2020) 169 [arXiv:1910.09384] [INSPIRE].

[120] B.I. Zwiebel, $N=4 S Y M$ to two loops: compact expressions for the non-compact symmetry algebra of the su(1,1|2) sector, JHEP 02 (2006) 055 [hep-th/0511109] [INSPIRE].

[121] Z. Xiao, BMN operators with a scalar fermion pair and operator mixing in $N=4$ Super Yang-Mills Theory, Phys. Rev. D 81 (2010) 026004 [arXiv:0910.3390] [inSPIRE].

[122] P. Caputa, C. Kristjansen and K. Zoubos, On the spectral problem of $N=4 S Y M$ with orthogonal or symplectic gauge group, JHEP 10 (2010) 082 [arXiv:1005.2611] [INSPIRE].

[123] V.N. Velizhanin, The non-planar contribution to the four-loop universal anomalous dimension in $N=4$ Supersymmetric Yang-Mills theory, JETP Lett. 89 (2009) 593 [arXiv:0902.4646] [INSPIRE].

[124] V.N. Velizhanin, The non-planar contribution to the four-loop anomalous dimension of twist-2 operators: first moments in $N=4 S Y M$ and non-singlet QCD, Nucl. Phys. B 846 (2011) 137 [arXiv: 1008.2752] [INSPIRE].

[125] V.N. Velizhanin, Non-planar anomalous dimension of twist-2 operators: higher moments at four loops, Nucl. Phys. B 885 (2014) 772 [arXiv:1404.7107] [INSPIRE]. 
[126] B. Eden, P. Heslop, G.P. Korchemsky and E. Sokatchev, Constructing the correlation function of four stress-tensor multiplets and the four-particle amplitude in $N=4 S Y M$, Nucl. Phys. B 862 (2012) 450 [arXiv:1201.5329] [InSPIRE].

[127] T. Fleury and R. Pereira, Non-planar data of $\mathcal{N}=4$ SYM, JHEP 03 (2020) 003 [arXiv: 1910.09428] [INSPIRE].

[128] B.A. Kniehl and V.N. Velizhanin, Nonplanar cusp and transcendental anomalous dimension at four loops in $\mathcal{N}=4$ supersymmetric Yang-Mills theory, Phys. Rev. Lett. 126 (2021) 061603 [arXiv:2010.13772] [INSPIRE].

[129] B. Eden, Y. Jiang, D. le Plat and A. Sfondrini, Colour-dressed hexagon tessellations for correlation functions and non-planar corrections, JHEP 02 (2018) 170 [arXiv:1710.10212] [INSPIRE].

[130] T. Bargheer, J. Caetano, T. Fleury, S. Komatsu and P. Vieira, Handling handles: nonplanar integrability in $\mathcal{N}=4$ supersymmetric Yang-Mills theory, Phys. Rev. Lett. 121 (2018) 231602 [arXiv: 1711.05326] [INSPIRE].

[131] B. Eden, Y. Jiang, M. de Leeuw, T. Meier, D. le Plat and A. Sfondrini, Positivity of hexagon perturbation theory, JHEP 11 (2018) 097 [arXiv: 1806. 06051] [INSPIRE].

[132] T. Bargheer, J. Caetano, T. Fleury, S. Komatsu and P. Vieira, Handling handles. Part II. Stratification and data analysis, JHEP 11 (2018) 095 [arXiv: 1809.09145] [INSPIRE].

[133] T. Bargheer, F. Coronado and P. Vieira, Octagons I: combinatorics and non-planar resummations, JHEP 08 (2019) 162 [arXiv: 1904.00965] [INSPIRE].

[134] W. Carlson, R. de Mello Koch and H. Lin, Nonplanar integrability, JHEP 03 (2011) 105 [arXiv: 1101.5404] [INSPIRE].

[135] R. de Mello Koch, B.A.E. Mohammed and S. Smith, Nonplanar integrability: beyond the SU(2) sector, Int. J. Mod. Phys. A 26 (2011) 4553 [arXiv:1106.2483] [inSPIRE].

[136] A. Sever, A.G. Tumanov and M. Wilhelm, Operator product expansion for form factors, Phys. Rev. Lett. 126 (2021) 031602 [arXiv:2009.11297] [INSPIRE].

[137] R. Ben-Israel, A.G. Tumanov and A. Sever, Scattering amplitudes - Wilson loops duality for the first non-planar correction, JHEP 08 (2018) 122 [arXiv: 1802.09395] [INSPIRE].

[138] Q. Jin, K. Ren and G. Yang, Two-loop anomalous dimensions of QCD operators up to dimension-sixteen and Higgs EFT amplitudes, arXiv:2011.02494 [INSPIRE]. 